\title{
\#USGS
}

science for a changing world

Prepared in cooperation with Eagle County, the Eagle River Water and Sanitation District, the Town of Eagle, the Town of Gypsum, and the Upper Eagle Regional Water Authority

\section{Groundwater Quality, Age, and Probability of Contamination, Eagle River Watershed Valley-Fill Aquifer, North-Central Colorado, 2006-2007}
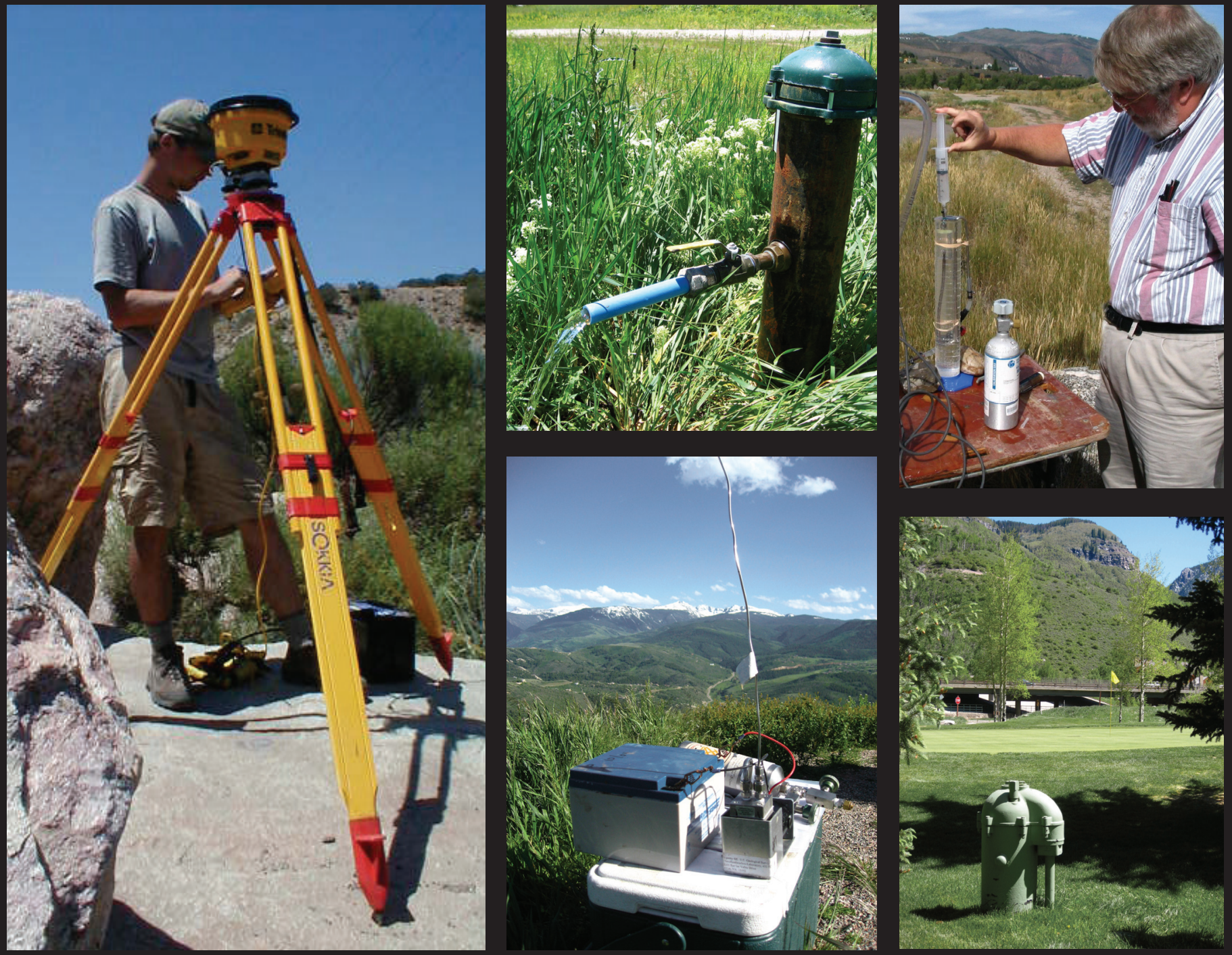

Scientific Investigations Report 2009-5082 


\section{FRONT COVER:}

Left: U.S. Geological Survey scientist collecting high-accuracy GPS data.

Top middle: Artesian well, Eagle County, Colorado.

Bottom middle: Collection of air sample near Bellyache Ridge, Eagle County, Colorado.

Top right: U.S. Geological Survey research hydrologist collecting groundwater age dating sample.

Bottom right: Water well, Eagle County, Colorado.

\section{BACK COVER:}

Sylvan Lake, Eagle County, Colorado.

All photographs by Michael G. Rupert, U.S. Geological Survey. 


\section{Groundwater Quality, Age, and Probability of Contamination, Eagle River Watershed Valley-Fill Aquifer, North-Central Colorado, 2006-2007}

By Michael G. Rupert and L. Niel Plummer

Prepared in cooperation with Eagle County, the Eagle River Water and Sanitation

District, the Town of Eagle, the Town of Gypsum, and the Upper Eagle Regional

Water Authority

Scientific Investigations Report 2009-5082 


\title{
U.S. Department of the Interior \\ KEN SALAZAR, Secretary
}

\author{
U.S. Geological Survey \\ Suzette M. Kimball, Acting Director
}

\section{U.S. Geological Survey, Reston, Virginia: 2009}

\begin{abstract}
For more information on the USGS - the Federal source for science about the Earth, its natural and living resources, natural hazards, and the environment, visit http://www.usgs.gov or call 1-888-ASK-USGS

For an overview of USGS information products, including maps, imagery, and publications, visit http://www.usgs.gov/pubprod

To order this and other USGS information products, visit http://store.usgs.gov
\end{abstract}

Any use of trade, product, or firm names is for descriptive purposes only and does not imply endorsement by the U.S. Government.

Although this report is in the public domain, permission must be secured from the individual copyright owners to reproduce any copyrighted materials contained within this report.

Suggested citation:

Rupert, M.G., and Plummer, L.N., 2009, Groundwater quality, age, and probability of contamination, Eagle River watershed valley-fill aquifer, north-central Colorado, 2006-2007: U.S. Geological Survey Scientific Investigations Report 2009-5082, 59 p. 


\section{Contents}

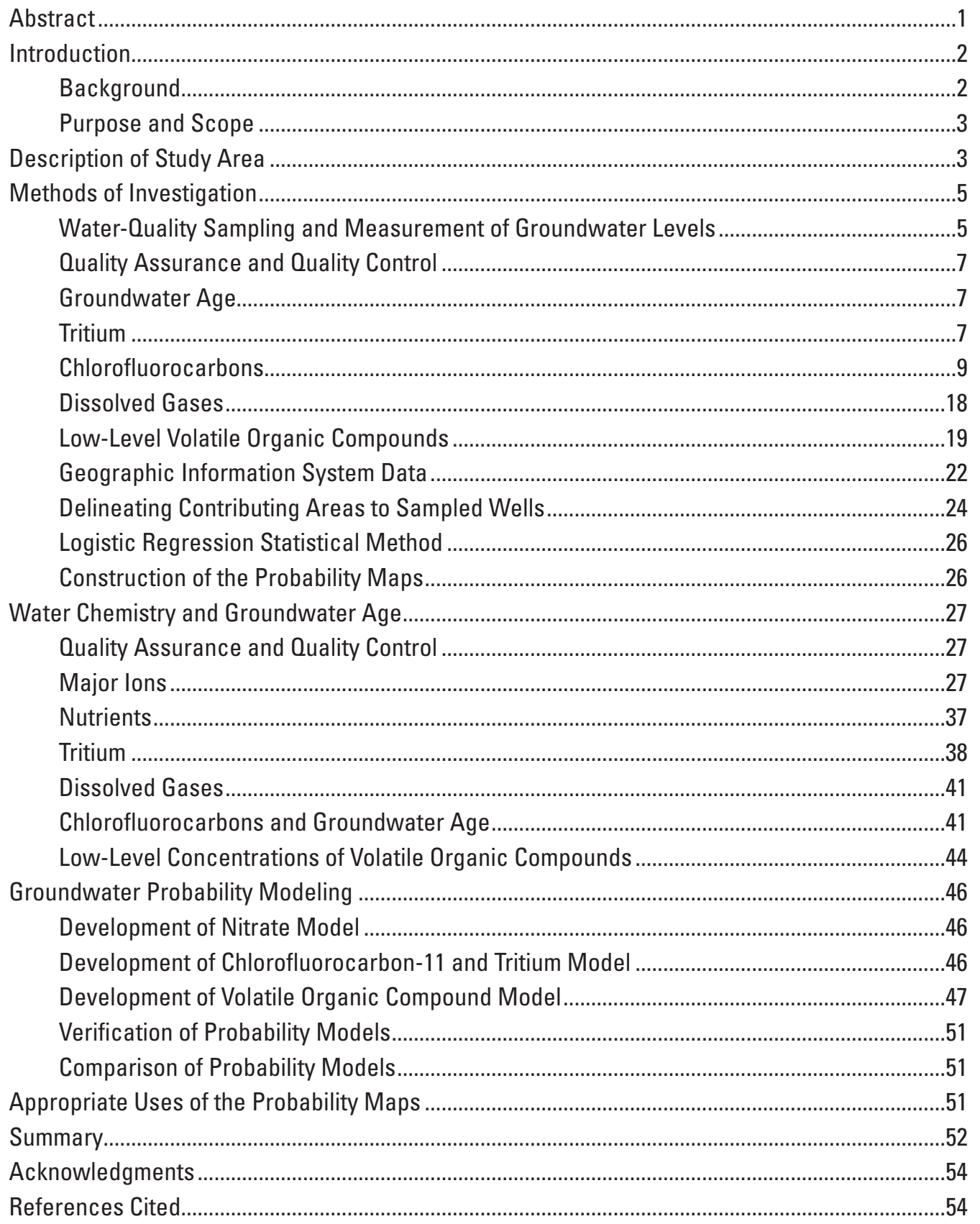




\section{Figures}

1. Map showing locations of surface-water bodies, towns, and the Eagle River watershed valley-fill aquifer, Eagle County, Colorado.

2. Map shownig local identification numbers of air, groundwater, and surfacewater sampling sites, Eagle River watershed, Eagle County, Colorado. 6

3. Graph showing concentrations of CFC -12 , CFC -11 , and CFC-113 in precipitation, and tritium activities in precipitation, 1940-2007.

4. Conceptual diagram of 90-degree pie-shaped buffer and circular buffer for delineating areas contributing recharge to a well.

5. Trilinear diagrams showing major-ion chemistry of surface-water-quality samples, Eagle River watershed, 2006-2007, Eagle County, Colorado.

6. Trilinear diagrams showing major-ion chemistry of ground- and surface-waterquality samples, Eagle River watershed, 2006-2007, Eagle County, Colorado.

7-10. Maps showing:

7. Sulfate concentrations in groundwater in the Eagle River watershed valley-fill aquifer, 2006-2007, Eagle County, Colorado

8. Total dissolved-solids concentrations in groundwater in the Eagle River watershed valley-fill aquifer, 2006-2007, Eagle County, Colorado

9. Locations of the National Atmospheric Deposition Program precipitation sites, north-central Colorado.

10. Nitrate concentrations in groundwater in the Eagle River watershed valley-

fill aquifer, 2006-2007, Eagle County, Colorado.

11. Graph showing boron, chloride, sulfate, and nitrate concentrations in groundwater, Eagle River watershed valley-fill aquifer, 2006-2007, Eagle County, Colorado

12. Graph showing measured CFC-11 concentrations and tritium activities in groundwater, Eagle River watershed valley-fill aquifer, 2006-2007, Eagle County, Colorado........40

13. Map showing mean recharge date of the young fraction of groundwater and the percentage of young groundwater from wells completed in the Eagle River watershed valley-fill aquifer, 2006-2007, Eagle County, Colorado.

14. Graph showing relations between total concentrations of halogenated volatile organic compounds with the total number of halogenated volatile organic compounds, Eagle River watershed, 2006-2007, Eagle County, Colorado

15-17. Maps showing:

15. Probability of detecting nitrate concentrations greater than 1 milligram per liter in the Eagle River watershed valley-fill aquifer, 2006-2007, Eagle County, Colorado.

16. Probability of detecting unmixed young water in the Eagle River watershed valley-fill aquifer (using chlorofluorocarbon-11 concentrations and tritium activities), 2006-2007, Eagle County, Colorado

17. Probability of detecting total volatile organic compound concentrations greater than 28,000 picograms per liter in the Eagle River watershed valley-fill aquifer, 2006-2007, Eagle County, Colorado

18. Graphs showing percentage of actual detections of nitrate greater than 1 milligram per liter, detections of unmixed young water, and detections of total volatile organic compounds greater than 28,000 picograms per liter, plotted with the predicted probability of detections of those compounds, Eagle River watershed valley-fill aquifer, Eagle County, Colorado. 


\section{Tables}

1. Locations of air, groundwater, and surface-water sampling sites, Eagle River watershed, 2006-2007, Eagle County, Colorado...

2. Selected well-construction, major-ion, nutrient, stable isotopes of hydrogen and oxygen, and tritium data from groundwater and surface water, Eagle River watershed, 2006-2007, Eagle County, Colorado

3. Selected ground- and surface-water-quality data used to interpret groundwater recharge dates using chlorofluorocarbons, Eagle River watershed, Eagle County, Colorado.

4. Mean concentrations of low-level volatile organic compounds detected in groundwater and surface water of the Eagle River watershed, 2006-2007, Eagle County, Colorado.

5. Concentrations, approximate retention times, and minimum detection levels of halogenated volatile organic compounds detected in the Eagle River watershed, 2006-2007, Eagle County, Colorado

6. Major-ion and nutrient analyses of blank samples collected from groundwater and surface water, Eagle River watershed, 2006-2007, Eagle County, Colorado, arranged chronologically.

7. Analyses of replicate samples of major ions, nutrients, stable isotopes of hydrogen and oxygen, and tritium collected from groundwater and surface water, Eagle River watershed, 2006-2007, Eagle County, Colorado, arranged chronologically

8. Summary statistics for major ions, nutrients, stable isotopes of hydrogen and oxygen, and tritium in groundwater, Eagle River watershed, 2006-2007, Eagle County, Colorado.

9. Summary statistics for ammonia and nitrate concentrations in precipitation measured by the National Atmospheric Deposition Program (2007) at the Niwot Saddle, Sand Spring, and Sunlight Peak sites, Colorado, 1988-2006

10. Dissolved-gas data analyzed from groundwater samples collected from the Eagle River watershed valley-fill aquifer, 2006-2007, Eagle County, Colorado

11. Chlorofluorocarbon and volatile organic compounds analyzed from air samples collected in the Eagle River watershed, 2006-2007, Eagle County, Colorado

12. Logistic regression modeling results, coefficients, and individual $p$-values of independent variables significantly related with the probability of nitrate concentrations greater than 1 milligram per liter, the probability of unmixed young water (using chlorofluorocarbon-11 concentrations and tritium activities), and the probability of total volatile organic compounds greater than 28,000 picograms per liter in the Eagle River watershed valley-fill aquifer, Eagle County, Colorado 


\section{Conversion Factors}

SI to Inch/Pound

\begin{tabular}{|c|c|c|}
\hline Multiply & By & To obtain \\
\hline \multicolumn{3}{|c|}{ Length } \\
\hline centimeter $(\mathrm{cm})$ & 0.3937 & inch (in.) \\
\hline millimeter (mm) & 0.03937 & inch (in.) \\
\hline meter $(\mathrm{m})$ & 3.281 & foot $(\mathrm{ft})$ \\
\hline kilometer $(\mathrm{km})$ & 0.6214 & mile (mi) \\
\hline \multicolumn{3}{|c|}{ Area } \\
\hline square meter $\left(\mathrm{m}^{2}\right)$ & 0.0002471 & acre \\
\hline square centimeter $\left(\mathrm{cm}^{2}\right)$ & 0.001076 & square foot $\left(\mathrm{ft}^{2}\right)$ \\
\hline hectare (ha) & 0.003861 & square mile $\left(\mathrm{mi}^{2}\right)$ \\
\hline square kilometer $\left(\mathrm{km}^{2}\right)$ & 0.3861 & square mile $\left(\mathrm{mi}^{2}\right)$ \\
\hline \multicolumn{3}{|c|}{ Volume } \\
\hline liter (L) & 0.2642 & gallon (gal) \\
\hline cubic meter $\left(\mathrm{m}^{3}\right)$ & 264.2 & gallon (gal) \\
\hline cubic centimeter $\left(\mathrm{cm}^{3}\right)$ & 0.06102 & cubic inch $\left(\mathrm{in}^{3}\right)$ \\
\hline liter $(\mathrm{L})$ & 61.02 & cubic inch $\left(\mathrm{in}^{3}\right)$ \\
\hline cubic meter $\left(\mathrm{m}^{3}\right)$ & 35.31 & cubic foot $\left(\mathrm{ft}^{3}\right)$ \\
\hline \multicolumn{3}{|c|}{ Flow rate } \\
\hline meter per second $(\mathrm{m} / \mathrm{s})$ & 3.281 & foot per second $(\mathrm{ft} / \mathrm{s})$ \\
\hline meter per day $(\mathrm{m} / \mathrm{d})$ & 3.281 & foot per day $(\mathrm{ft} / \mathrm{d})$ \\
\hline meter per year $(\mathrm{m} / \mathrm{yr})$ & 3.281 & foot per year $\mathrm{ft} / \mathrm{yr}$ ) \\
\hline cubic meter per day $\left(\mathrm{m}^{3} / \mathrm{d}\right)$ & 35.31 & cubic foot per day $\left(\mathrm{ft}^{3} / \mathrm{d}\right)$ \\
\hline cubic meter per day $\left(\mathrm{m}^{3} / \mathrm{d}\right)$ & 264.2 & gallon per day (gal/d) \\
\hline millimeter per year $(\mathrm{mm} / \mathrm{yr})$ & 0.03937 & inch per year (in/yr) \\
\hline \multicolumn{3}{|c|}{ Mass } \\
\hline gram $(\mathrm{g})$ & 0.03527 & ounce, avoirdupois (oz) \\
\hline kilogram (kg) & 2.205 & pound avoirdupois (lb) \\
\hline \multicolumn{3}{|c|}{ Radioactivity } \\
\hline becquerel per liter $(\mathrm{Bq} / \mathrm{L})$ & 27.027 & picocurie per liter $(\mathrm{pCi} / \mathrm{L})$ \\
\hline \multicolumn{3}{|c|}{ Hydraulic conductivity } \\
\hline meter per day $(\mathrm{m} / \mathrm{d})$ & 3.281 & foot per day $(\mathrm{ft} / \mathrm{d})$ \\
\hline \multicolumn{3}{|c|}{ Hydraulic gradient } \\
\hline meter per kilometer $(\mathrm{m} / \mathrm{km})$ & 5.27983 & foot per mile (ft/mi) \\
\hline \multicolumn{3}{|c|}{ Transmissivity* } \\
\hline meter squared per day $\left(\mathrm{m}^{2} / \mathrm{d}\right)$ & 10.76 & foot squared per day $\left(\mathrm{ft}^{2} / \mathrm{d}\right)$ \\
\hline
\end{tabular}

Temperature in degrees Celsius $\left({ }^{\circ} \mathrm{C}\right)$ may be converted to degrees Fahrenheit $\left({ }^{\circ} \mathrm{F}\right)$ as follows:

$$
{ }^{\circ} \mathrm{F}=\left(1.8 \mathrm{x}^{\circ} \mathrm{C}\right)+32
$$

Temperature in degrees Fahrenheit $\left({ }^{\circ} \mathrm{F}\right)$ may be converted to degrees Celsius $\left({ }^{\circ} \mathrm{C}\right)$ as follows:

$$
{ }^{\circ} \mathrm{C}=\left({ }^{\circ} \mathrm{F}-32\right) / 1.8
$$

Vertical coordinate information is referenced to the North American Vertical Datum of 1988 (NAVD 88).

Horizontal coordinate information is referenced to the North American Datum of 1983 (NAD 83). Altitude, as used in this report, refers to distance above the vertical datum.

*Transmissivity: The standard unit for transmissivity is cubic foot per day per square foot times foot of aquifer thickness [(ft $\left.\left.\mathrm{ft}^{3} \mathrm{~d}\right) / \mathrm{ft}^{2}\right] \mathrm{ft}$. In this report, the mathematically reduced form, foot squared per day $\left(\mathrm{ft}^{2} / \mathrm{d}\right)$, is used for convenience.

Specific conductance is given in microsiemens per centimeter at 25 degrees Celsius $(\mu \mathrm{S} / \mathrm{cm}$ at $\left.25^{\circ} \mathrm{C}\right)$.

Concentrations of chemical constituents in water are given either in milligrams per liter (mg/L) or micrograms per liter $(\mu \mathrm{g} / \mathrm{L})$. 


\section{Acronyms and Abbreviations}

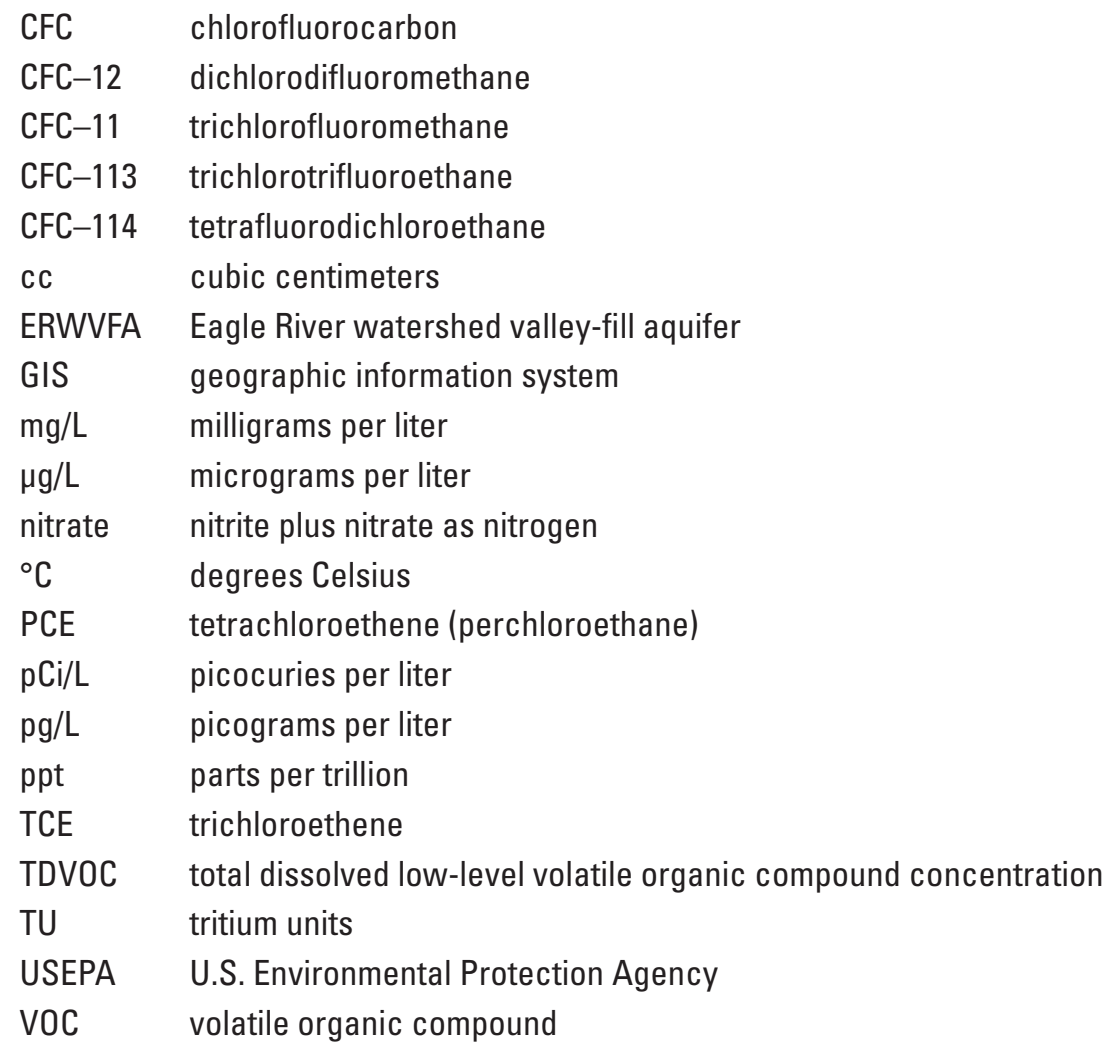




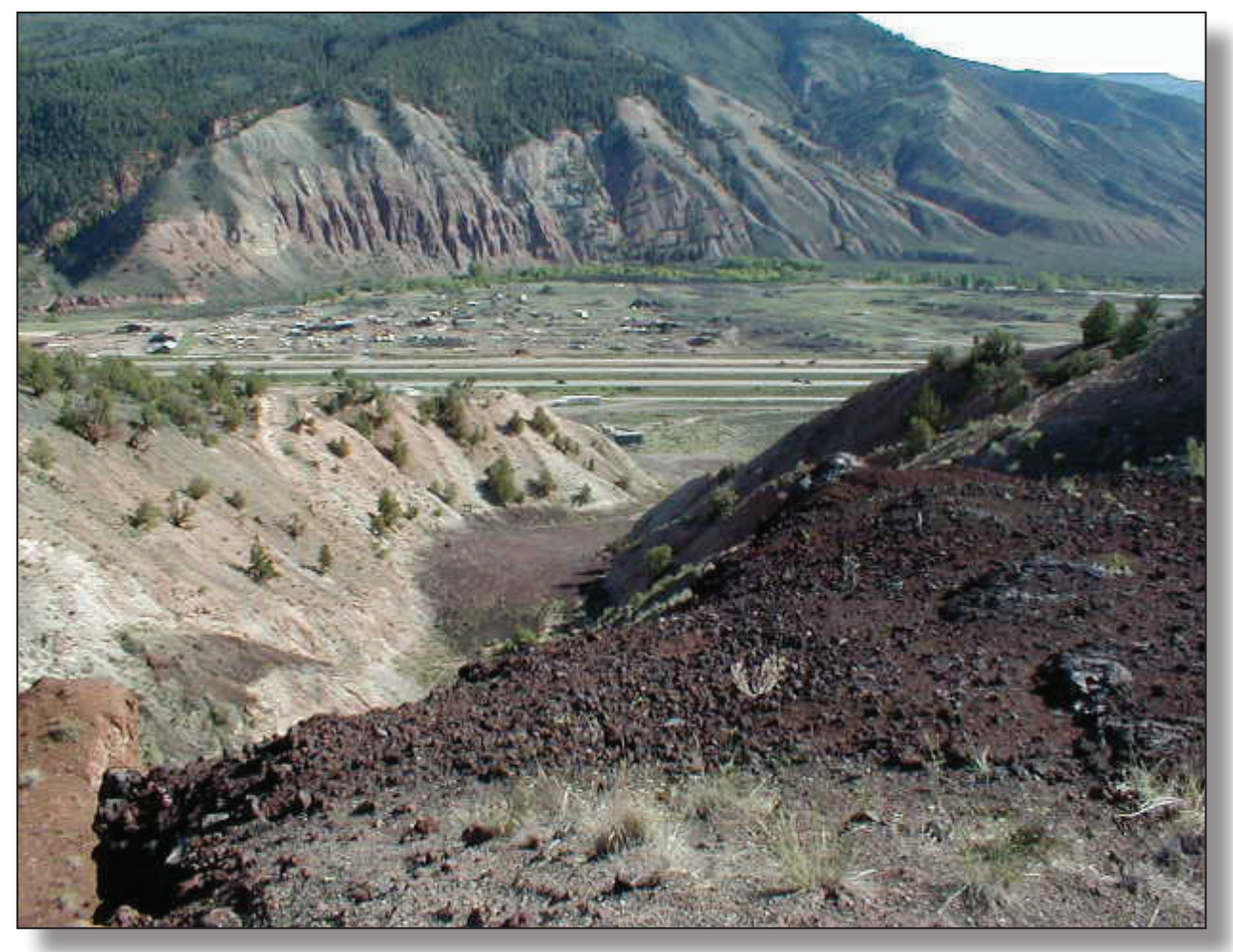

The most recent basalt flow in Colorado (near Dotsero).

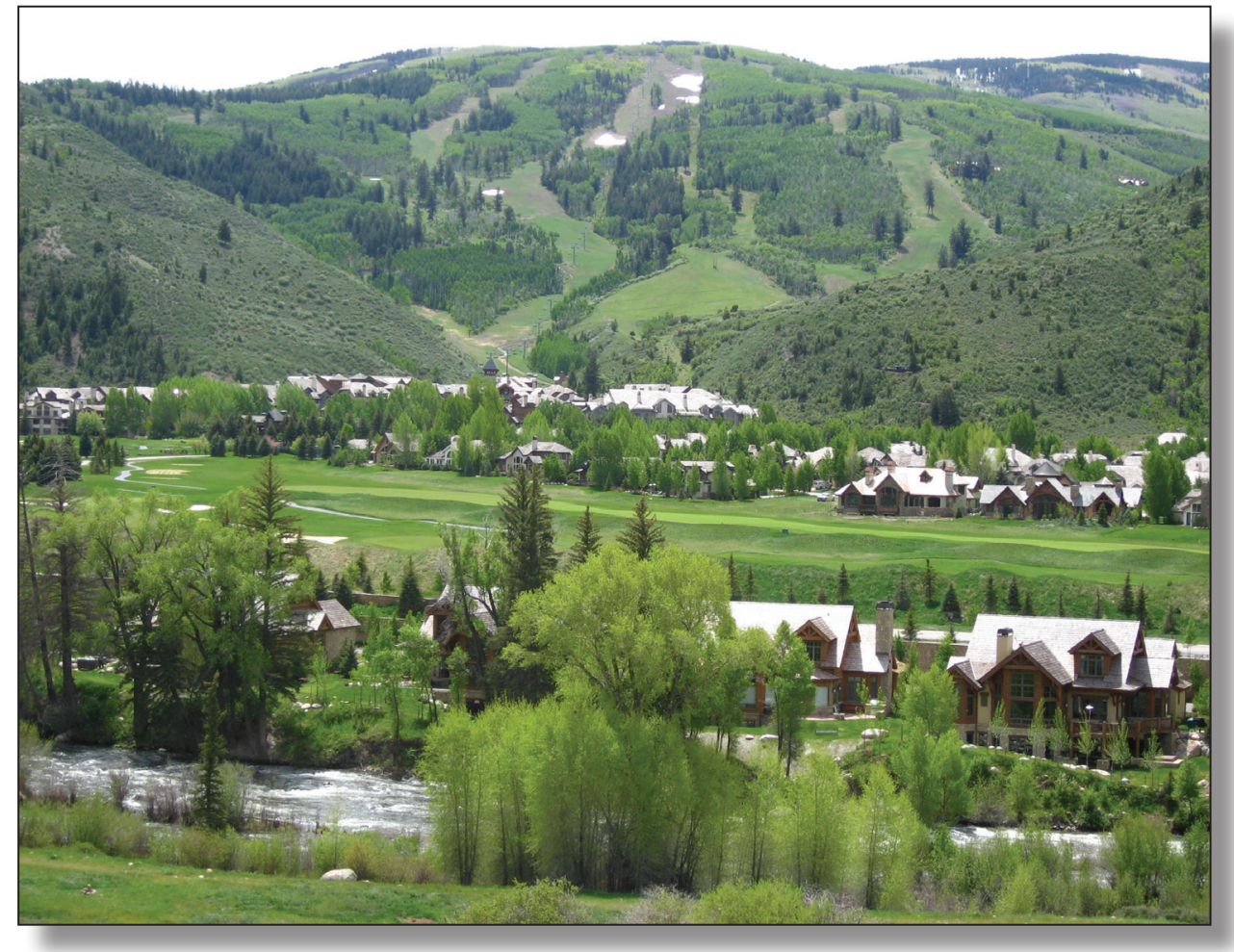

Looking south towards Arrowhead ski area (just west of Beaver Creek). 


\title{
Groundwater Quality, Age, and Probability of Contamination, Eagle River Watershed Valley-Fill Aquifer, North-Central Colorado, 2006-2007
}

\author{
By Michael G. Rupert and L. Niel Plummer
}

\section{Abstract}

The Eagle River watershed is located near the destination resort town of Vail, Colorado. The area has a fastgrowing permanent population, and the resort industry is rapidly expanding. A large percentage of the land undergoing development to support that growth overlies the Eagle River watershed valley-fill aquifer (ERWVFA), which likely has a high predisposition to groundwater contamination. As development continues, local organizations need tools to evaluate potential land-development effects on ground- and surfacewater resources so that informed land-use and water management decisions can be made. To help develop these tools, the U.S. Geological Survey (USGS), in cooperation with Eagle County, the Eagle River Water and Sanitation District, the Town of Eagle, the Town of Gypsum, and the Upper Eagle Regional Water Authority, conducted a study in 2006-2007 of the groundwater quality, age, and probability of contamination in the ERWVFA, north-central Colorado.

Ground- and surface-water quality samples were analyzed for major ions, nutrients, stable isotopes of hydrogen and oxygen in water, tritium, dissolved gases, chlorofluorocarbons (CFCs), and volatile organic compounds (VOCs) determined with very low-level laboratory methods. The major-ion data indicate that groundwaters in the ERWVFA can be classified into two major groups: groundwater that was recharged by infiltration of surface water, and groundwater that had less immediate recharge from surface water and had elevated sulfate concentrations. Sulfate concentrations exceeded the USEPA National Secondary Drinking Water Regulations (250 milligrams per liter) in many wells near Eagle, Gypsum, and Dotsero. The predominant source of sulfate to groundwater in the Eagle River watershed is the Eagle Valley Evaporite, which is a gypsum deposit of Pennsylvanian age located predominantly in the western one-half of Eagle County.

Nitrite plus nitrate as nitrogen (nitrate) concentrations in groundwater in the ERWVFA were generally low, with the median nitrate concentration about 0.74 milligram per liter $(\mathrm{mg} / \mathrm{L})$ and a maximum concentration of $5.4 \mathrm{mg} / \mathrm{L}$, which is less than the USEPA Maximum Contaminant Level of $10 \mathrm{mg} / \mathrm{L}$. More than 50 percent of the nitrate concentrations in the ERWVFA were less than $1 \mathrm{mg} / \mathrm{L}$, indicating that more than 50 percent of the wells tested in the ERWVFA had nitrate concentrations similar to precipitation. Most groundwater in the ERWVFA was under oxidized geochemical conditions, indicating that nitrate from anthropogenic sources (caused or produced by humans) could persist for several decades in groundwater of the ERWVFA.

The groundwater age-dating data indicated that most groundwater in the ERWVFA was recently recharged water and had a high probability of contamination if anthropogenic compounds were released to the environment. Based upon the CFC concentrations and tritium activities in groundwater, the median groundwater recharge date was 1989 and the standard deviation was about 9 years, indicating that most groundwater in the ERWVFA that was sampled was young water. The median percentage of young water was 83 percent, and the standard deviation was about 28 percent, indicating that only a small portion of water from most wells was composed of old (older than 1940) water.

VOCs were detected in all water samples at or above the low-level laboratory reporting limit concentrations, but VOC concentrations in all samples were at least one order of magnitude less than their USEPA Maximum Contaminant Level. Some of those VOCs can be naturally occurring at these extremely low concentrations. Total VOC concentrations above 28,000 picograms per liter were attributed to anthropogenic sources.

Logistic regression statistical modeling techniques were used to develop statistical models that predict the probability of elevated nitrate concentrations, the probability of unmixed young water (using chlorofluorocarbon-11 concentrations and tritium activities), and the probability of elevated VOC concentrations. These three models used different compounds such as nitrate and VOCs to provide an indication of the probability of groundwater contamination under a variety of conditions and contaminant inputs. The statistical parameters produced by the three logistic regression models indicated they were highly significant models; 
McFadden's rho ranged between 0.490 and 0.647 and the total correct predictions ranged between 0.778 and 0.876 . The logistic regression models were verified by plotting the percentage of actual detections with the predicted probability of detections using a deciles of risk calculation. R-squared values of plots of actual detections with the predicted probability of detections were between 0.995 and 0.998 , verifying they are highly effective models. Although the groundwater age dating indicates that most areas of the ERWVFA have a high probability of contamination, the probability maps help to show areas with a particularly high probability of contamination if compounds of concern are released to the environment.

\section{Introduction}

The Eagle River watershed is located near the destination resort town of Vail, Colorado. The area has a fast-growing permanent population, and the resort industry is rapidly expanding. The permanent population has increased 23 percent between April 1, 2000, and July 1, 2007 (U.S. Census Bureau, 2008). The popularity of the area is largely due to the presence of relatively pristine environmental conditions that the community wishes to protect. Most of the land slated for future development within the watershed is immediately adjacent to the Eagle River or streams that are tributary to the Eagle River and overlies the Eagle River watershed valley-fill aquifer (ERWVFA), which likely has a high predisposition to groundwater contamination. As development continues, local governments need tools to manage existing environmental concerns and to evaluate potential landdevelopment effects on ground- and surface-water resources. For instance, local governments need information to help them evaluate the most suitable locations for light industry such as automobile repair shops and dry cleaners to help protect the groundwater resources from contamination. It is not known what effect onsite wastewater-disposal (septic) systems will have on the groundwater quality. Tools are needed to identify areas with the highest predisposition to groundwater contamination so that wise land-use and source-water protection decisions can be made. To help meet these needs, the U.S. Geological Survey (USGS), in cooperation with Eagle County, the Eagle River Water and Sanitation District, the Town of Eagle, the Town of Gypsum, and the Upper Eagle Regional Water Authority, conducted a study in 2006-2007 of the groundwater quality, age, and probability of contamination in the Eagle River watershed, north-central Colorado. The goals of this study were to (1) establish baseline conditions for major ions and nutrients by summarizing groundwater quality data, (2) develop maps showing the concentrations of sulfate, total dissolved solids, and nitrate in groundwater of the ERWVFA, (3) determine the age of the groundwater in the ERWVFA, and (4) develop maps to show the probability of groundwater in the ERWVFA to contamination.

\section{Background}

Maps showing the predisposition to groundwater contamination are commonly referred to as "groundwater vulnerability maps," "groundwater susceptibility maps," or "groundwater probability maps." Different definitions of groundwater vulnerability have arisen over time. The first, and probably the most widely known groundwater vulnerability mapping procedure, is DRASTIC (Aller and others, 1985). The DRASTIC acronym refers to the seven hydrogeologic factors considered in the model: depth to water, net recharge, aquifer media, soil media, topography, impact of vadose (unsaturated) zone media, and hydraulic conductivity of the aquifer (Aller and others, 1985, p. iv). DRASTIC used the term "vulnerability" in a generic sense, referring to the overall potential for nonpoint-source groundwater contamination with no distinction to the general classes of data layers that would be included in the map. More recently, researchers defined groundwater vulnerability as the combination of hydrogeologic susceptibility plus the input of contaminants to the land surface and delivery to the groundwater (National Research Council, 1993; Rao and Alley, 1993; U.S. Environmental Protection Agency, 1993; Vowinkel and others, 1996; Focazio and others, 2002; Gurdak and Qi, 2006). The hydrogeologic susceptibility is determined by evaluating how hydrogeologic properties such as depth to groundwater, geology, and soils influence groundwater contamination; vulnerability is the combination of susceptibility plus contaminant input. The models developed in this report can be considered susceptibility models because they are based on hydrogeologic properties.

The DRASTIC procedure has been used to develop groundwater vulnerability maps in many parts of the Nation, but the validity and accuracy of the model has had poor success because DRASTIC maps use a vulnerability point-rating system that is based upon best professional judgment instead of calibration of the point-rating system to actual contaminant concentrations (Koterba and others, 1993, p. 513; Barbash and Resek, 1996; Rupert, 2001). Rupert (2001) improved the validity and accuracy of a modified DRASTIC vulnerability map by calibrating (adjusting) the vulnerability point ratings to measured nitrite plus nitrate as nitrogen (nitrate) concentrations in groundwater using nonparametric statistical tests.

The validity and accuracy of groundwater vulnerability maps have been further improved by using statistical modeling methods such as logistic regression to relate water-quality data to anthropogenic (caused or produced by humans) and hydrogeologic factors (Koterba and others, 1993; Druliner and others, 1996; Nolan and Clark, 1997; Tesoriero and Voss, 1997; Rupert, 1998, 2003; Nolan and Hitt, 2006). Logistic regression (Hosmer and Lemeshow, 1989; Kleinbaum, 1994) is a statistical method that can be used to predict the probability of occurrence of an event of interest (such as detection of a contaminant in groundwater) as a function of a set of independent variables (such as land cover and soils). Maps developed using logistic regression are sometimes called probability maps because (1) the maps are based on the results of statistical correlations with groundwater 
quality, and (2) the contamination potential is stated in the terms of percent probability of a detection (Rupert, 1998, 2003). Groundwater probability maps developed using logistic regression are more accurate predictive tools (Rupert, 2003) than vulnerability maps developed with the DRASTIC mapping technique because they are statistically derived from actual contaminant concentrations, and the coefficients of the explanatory variables are automatically adjusted during model calibration.

\section{Purpose and Scope}

This report describes groundwater quality, age, and probability of contamination of the ERWVFA. The primary purpose of this report is to develop maps that show the probability of groundwater in the ERWVFA to contamination by using hydrogeologic factors such as depth to groundwater and soil characteristics. These maps are intended to help provide a sound hydrogeologic basis for the protection of groundwater in the ERWVFA and provide a tool to help resource managers to prioritize areas for groundwater-quality monitoring or implement alternative management practices. To meet the primary purpose of this report, different compounds were used to calibrate the groundwater probability maps than compounds that have been used in other areas of the Nation. Development in Eagle County has happened, for the most part, during the last 20 years or so (U.S. Census Bureau, 2008), and new land development in Eagle County is urban and not agricultural. Instead of using agriculturally derived contaminants such as atrazine, concentrations of nitrate and groundwater age-dating compounds were used to calibrate the logistic regression models under the assumption that recently recharged groundwaters have a greater predisposition to contamination than waters recharged many years earlier. Concentrations of volatile organic compounds (VOCs) determined with very low-level laboratory methods and nitrate were also used to calibrate logistic regression models because VOCs and nitrate are common urban contaminants. This report evaluated the ERWVFA; the surrounding bedrock areas were beyond the scope of this report because of the lack of sufficient hydrogeologic information.

\section{Description of Study Area}

The ERWVFA is in north-central Colorado, in Eagle County (fig. 1). The ski resort town of Vail is adjacent to the ERWVFA. Annual precipitation ranges from $915 \mathrm{~mm}$ (36 inches) in the surrounding mountains to less than $254 \mathrm{~mm}$ (10 inches) near Dotsero, on the western edge of the study area (Daly and others, 1994). Land-surface elevations of the ERWVFA range from about 2,620 m (8,600 ft) east of Vail to about 1,860 m (6,100 ft) west of Dotsero. Land-surface elevations of the surrounding mountains can exceed 3,960 m (13,000 ft) (Apodaca and others, 1996).
The ERWVFA is located in the White River Uplift (Apodaca and others, 1996), where the geology consists mostly of sedimentary rocks of Mesozoic and Paleozoic age (Lidke, 1998), which include the Leadville Limestone and Eagle Valley Evaporite. Ground- and surface-water quality in the central and western reaches of the Eagle River watershed is influenced by dissolution of gypsum and other salts from the Eagle Valley Evaporite (Apodaca and others, 1996). Quaternary- and Tertiary-age volcanic rocks occur in localized areas; one of the youngest basalt flows in Colorado is located just east of Dotsero and was dated at about 4,300 years before present (Giegengack, 1962).

Rangeland and forest lands account for the largest land area in Eagle County (Apodaca and others, 1996). Livestock (sheep and cattle) use large areas of rangeland and forest for foraging. Forest land that includes most of the mountain areas is used for some commercial lumber production from lodgepole pine, Englemann spruce, and Douglas fir. Forest land also provides wildlife habitat and recreational opportunities. Tourism and recreational activities are a major industry in the study area. Urban land use is expanding near the towns of Vail, Avon, Eagle, Gypsum, and the unincorporated area of Edwards, in response to the development by the ski and tourism industry.

Mining is another important land use in the study area. Past and present mining activities have included the extraction of metals (copper, gold, lead, molybdenum, nickel, silver, vanadium, and zinc) and gypsum (Apodaca and others, 1996). The Eagle mine, located near Minturn, is designated as a Superfund site because of contamination by heavy metals such as arsenic, cadmium, lead, manganese, and zinc associated with the mining wastes from past mining activities. Gypsum is currently (2008) being mined and processed into drywall at a plant and mine located near Gypsum, Colorado.

The study area is located in three ecoregions: high-altitude tundra, low- to high-altitude forests, and low- to middle-altitude semidesert shrublands (Apodaca and others, 1996). Ecoregions are grouped into similar areas based on landscape features such as vegetation, soils, geology, physiography, and land use (Gallant and others, 1989). High-altitude tundra has a cold, humid to arid climate with vegetation above treeline containing low-growth shrubs, cushion plants, and forbs (Apodaca and others, 1996). Along the forest/tundra interface, there are sparse stands of Engelmann spruce, subalpine fir, limber pine, and bristlecone pine. The middle- to high-altitude forests consist of Engelmann spruce and subalpine fir; some areas are locally dominated by aspen. These forests vary from cool humid to warm dry climates, depending on the altitude. In the low- to middle-altitude forests, vegetation consists of aspen, Douglas fir, ponderosa pine, gambel oak, and piñon pine-juniper woodlands. Low- to middle-altitude semidesert shrublands have a semiarid climate with vegetation consisting of greasewood, four-winged saltbrush, shadscale, and sagebrush often interspersed with grasses. 


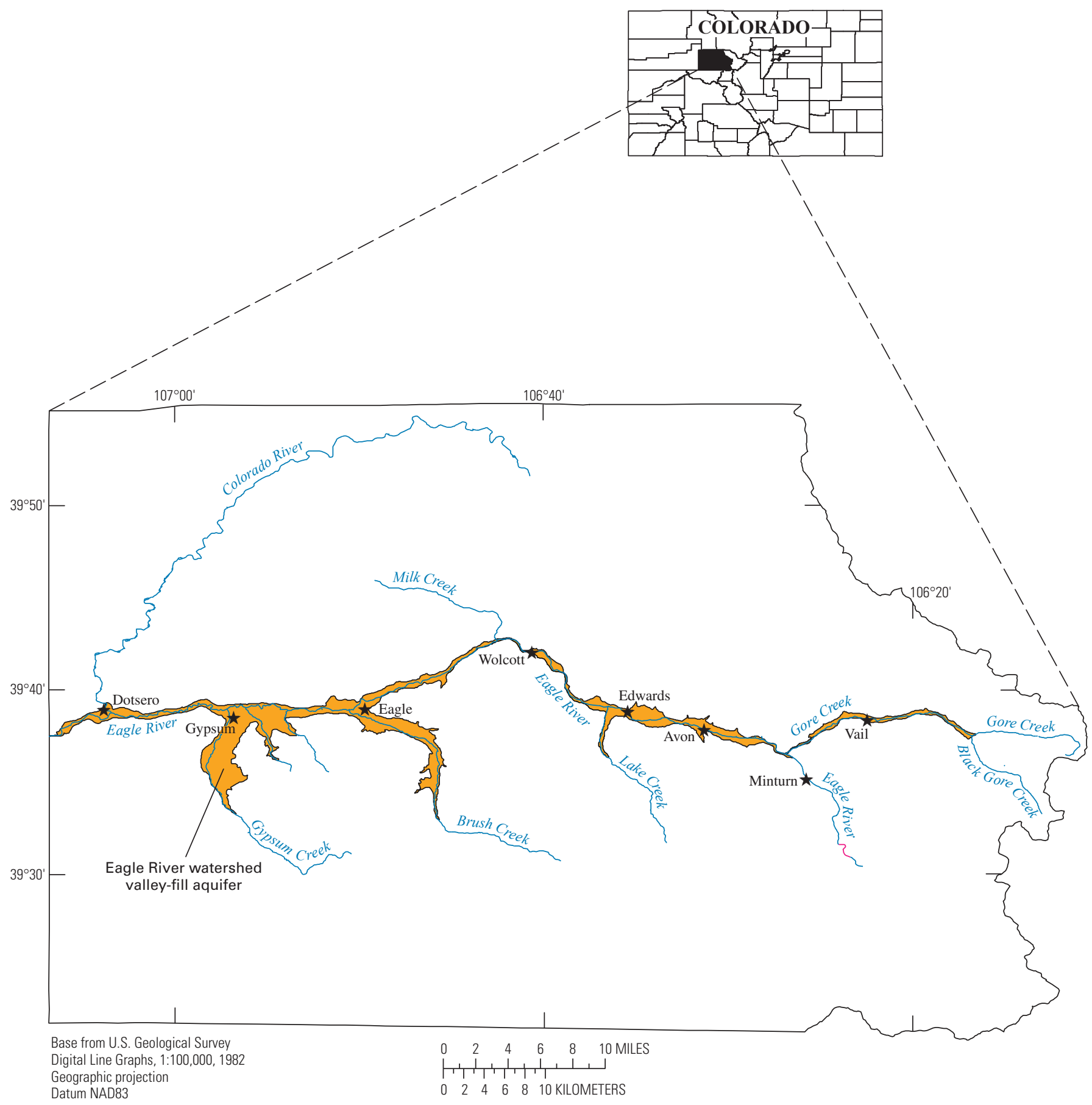

Figure 1. Locations of surface-water bodies, towns, and the Eagle River watershed valley-fill aquifer, Eagle County, Colorado. 


\section{Methods of Investigation}

The following methods were used to meet the purposes of this report. Ground- and surface-water quality data were collected to establish baseline conditions for major ions and nutrients and to develop maps showing the concentrations of sulfate, total dissolved solids, and nitrate in groundwater. Groundwater-level measurements were made to provide data for a depth-to-groundwater map, which was incorporated into one of the groundwater probability maps. Chlorofluorocarbons and tritium were measured in groundwater and surface water to provide data for groundwater age dating. Low-level concentrations of VOCs were measured in groundwater and surface water to serve as one of the tracers used in one of the groundwater probability maps. Geographic Information System (GIS) data on geology, hydrography, land-surface elevations, precipitation, and soils were compiled to provide input data for the groundwater probability models. Upgradient 90-degree pie-shaped zones of influence were calculated for each well where groundwater-quality data were collected to determine the average soils properties upgradient from each sampled well, which were then incorporated into the input data sets for the logistic regression modeling. The optimum size for each pie-shaped zone was calculated using groundwater modeling and particle tracking. Three statistical models predicting the probability of groundwater contamination by various contaminants were developed using logistic regression techniques. These three models predict the probability of elevated nitrate concentrations in groundwater of the ERWVFA, the probability of unmixed young water (using chlorofluorocarbon-11 concentrations and tritium activities) in groundwater of the ERWVFA, and the probability of elevated VOCs in groundwater of the ERWVFA.

\section{Water-Quality Sampling and Measurement of Groundwater Levels}

From existing wells and surface-water sites, 61 sites for groundwater-quality sampling and 10 sites for surfacewater-quality sampling were selected to provide appropriate areal coverage in the study area to meet study objectives. Well depths ranged from 17 to 543 feet. Groundwater-quality samples were collected once from 10 sites during August 2006 and once from an additional 51 sites (fig. 2, tables 1 and 2) during May through June 2007 and analyzed for major ions, nutrients, stable isotopes of hydrogen and oxygen $\left({ }^{2} \mathrm{H}\right.$ and ${ }^{18} \mathrm{O}$ ) in water, tritium, dissolved gases, chlorofluorocarbons (CFCs), and VOCs determined with very low-level laboratory methods (tables 2-4). Surface-water-quality samples were collected quarterly from six sites between October 2006 and September 2007 and analyzed for major ions, nutrients, ${ }^{2} \mathrm{H}$ and ${ }^{18} \mathrm{O}$ in water, and tritium (fig. 2, table 2). Additional samples were collected from the six surface-water sites, plus four additional sites, during high flow (May 2007) and low flow (August 2006 and August 2007) and analyzed for CFCs and VOCs (tables 3-4).

Water levels in wells were measured manually at the time of sampling by using a calibrated electric tape or steel tape. To prevent cross-contamination of bacteria between wells, the electric and steel tapes were disinfected immediately after measuring the water level by using a mild bleach bath followed by a deionized water rinse, according to State of Colorado regulations (State of Colorado, 2005).

Water samples were collected from wells and streams using procedures described by Koterba and others (1995), the USGS National Field Manual (U.S. Geological Survey, variously dated), and the USGS Chlorofluorocarbon and Stable Isotope Laboratories (U.S. Geological Survey, 2007a, 2007b). Water was pumped from domestic and municipal wells using existing pumps, and samples were collected prior to any pressure tanks, filtering, or treatment devices. Water was pumped from monitoring wells with a submersible electric pump constructed of stainless steel and Teflon. Water samples were processed onsite in a mobile laboratory by using methods designed to minimize changes to the water-sample chemistry. Prior to sample collection, stagnant water was flushed from the well by purging at least three casing volumes from the well. While purging, specific conductance, $\mathrm{pH}$, water temperature, and dissolved oxygen were measured until readings were stable. Once readings had stabilized, water samples were collected in precleaned bottles within an enclosed chamber to prevent sample contamination. To prevent degradation of water samples and maintain the initial concentration of compounds between the time of sample collection and laboratory analyses, bottles were preserved according to the requirements of the laboratories. Preservation practices differ among analytes and may include chilling, filtration ( 0.45 micrometer), and(or) chemical treatment (U.S. Geological Survey, variously dated). Sampling equipment was cleaned following the collection of samples using procedures listed in the USGS National Field Manual (U.S. Geological Survey, variously dated).

Samples for alkalinity and hydrogen sulfide were analyzed in the field by using procedures listed in the USGS National Field Manual (U.S. Geological Survey, variously dated). Samples for major ions and nutrients were analyzed by the USGS National Water Quality Laboratory in Lakewood, Colorado (Fishman, 1993; Fishman and Friedman, 1989). Samples for ${ }^{2} \mathrm{H}$ and ${ }^{18} \mathrm{O}$ in water were analyzed by the USGS Stable Isotope Laboratory in Reston, Virginia (U.S. Geological Survey, 2007b; Coplen and others, 1991; Epstein and Mayeda, 1953). Tritium activities were measured at the USGS Tritium Laboratory in Menlo Park, California (Thatcher and others, 1977). Samples for CFCs and very low concentrations of VOCs were analyzed by the USGS Chlorofluorocarbon Laboratory in Reston, Va. (Plummer and Busenberg, 1999). 
EAST HALF OF THE EAGLE RIVER WATERSHED VALLEY-FILL AQUIFER

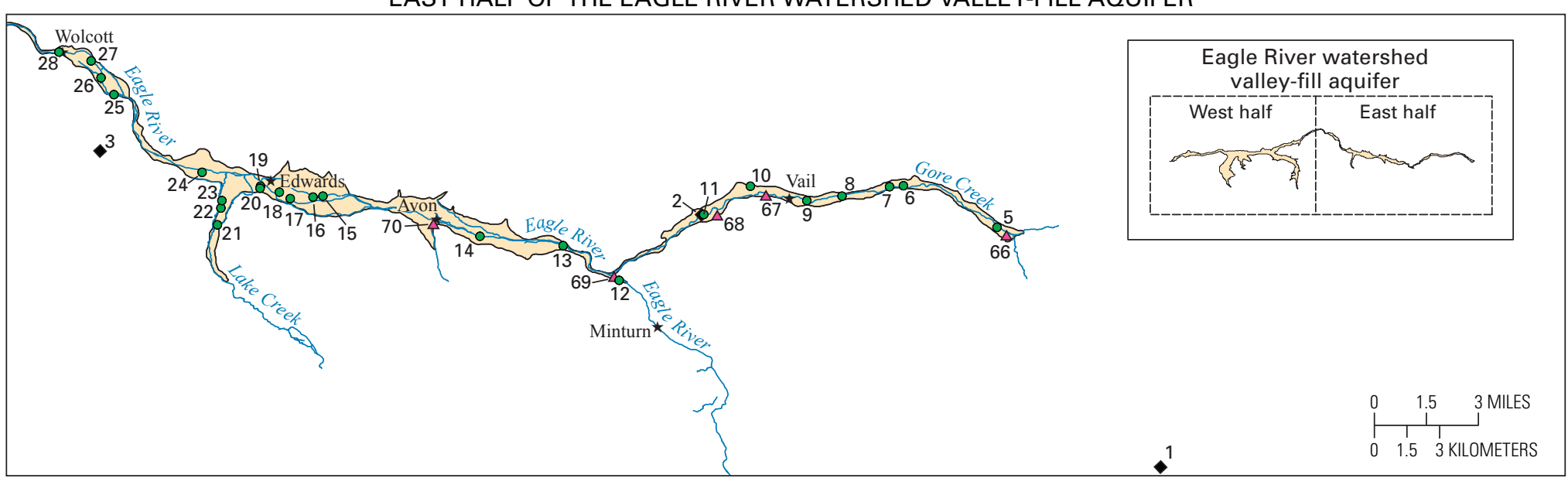

WEST HALF OF THE EAGLE RIVER WATERSHED VALLEY-FILL AQUIFER

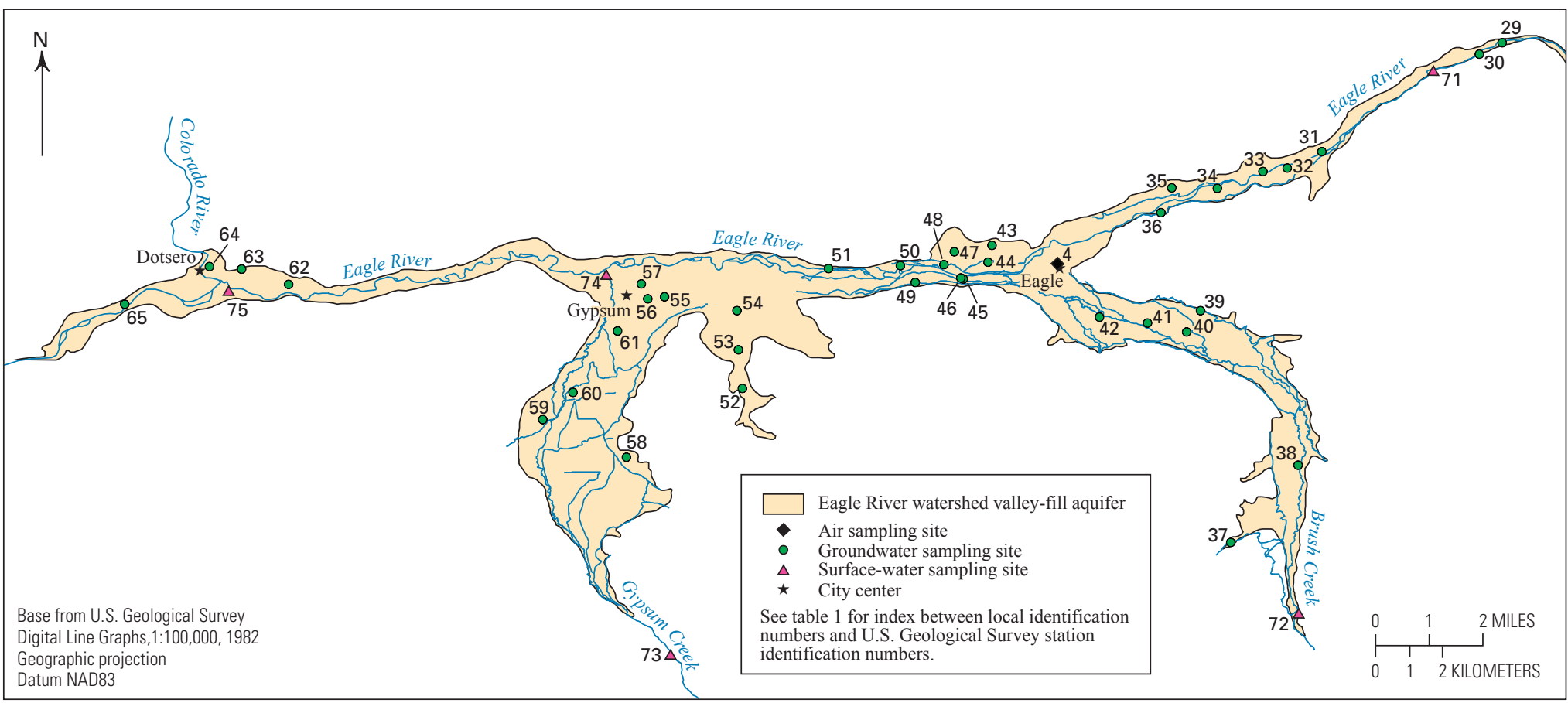

Figure 2. Local identification numbers of air, groundwater, and surface-water sampling sites, Eagle River watershed, Eagle County, Colorado. 


\section{Quality Assurance and Quality Control}

Field blank and replicate samples were collected at a rate of approximately 1 out of every 10 (10 percent) samples collected at ground- and surface-water sites. Field blanks were collected to determine if decontamination of the sampling equipment between sites is adequate and if sample contamination occurred during sample collection, transport, and analysis. All field blanks were collected using blank water prepared at the USGS National Water-Quality Laboratory that is certified to be free of environmental sample analytes above their reporting levels. Field blanks were processed using the same procedures and equipment as the environmental samples. Field blanks were analyzed for major ions and nutrients. It is not possible to prepare meaningful blank solutions for ${ }^{2} \mathrm{H}$ and ${ }^{18} \mathrm{O}$, tritium, dissolved gases, CFCs, and very low concentrations of VOCs, so no field blanks for these compounds were collected.

Replicate samples were collected to assess variability due to the collection and analyses of samples. All replicates were sequential; the replicate sample was collected after the native sample and analyzed using the same method. Replicate analyses were done for major ions, nutrients, ${ }^{2} \mathrm{H}$ and ${ }^{18} \mathrm{O}$, and tritium. Replicate samples for dissolved gases are collected at each site as part of standard sample collection, so additional dissolved gas replicate samples were not collected. Five replicate CFC and VOC samples were collected at each site as part of standard sample collection, so additional CFC and VOC samples were not collected.

\section{Groundwater Age}

Groundwater ages commonly are termed "apparent age" because the ages are modeled using simplifying assumptions regarding transport processes that may affect the age-dating constituents (such as CFCs) in the water (Plummer and Busenberg, 1999). The simplest and most common transport assumption in groundwater age dating is to assume piston flow, which assumes that the constituent concentration was not altered by transport processes (such as mixing or dispersion) from the point of entry to the measurement point in the aquifer. Some groundwater ages determined with a pistonflow model may be an oversimplification because mixing and dispersion can occur during groundwater flow.

All groundwater pumped from wells is, to some extent, water mixed within the well bore (Plummer and Busenberg, 1999). It is common to have the youngest waters near the top of an aquifer (near the source of recharge) and the oldest waters near the bottom of an aquifer. If the well screen is long, water flowing into a well can be drawn from multiple portions of the aquifer, where it mixes within the well bore. Mixing of water in the well bore produces mixed ages, which can complicate modeling of apparent age. Apparent ages of water sampled from narrow intervals of an aquifer probably are affected less by mixing than those ages interpreted from water samples pumped from large open intervals or from well bores open to multiple fractures of water-bearing zones; however, water from wells with open intervals as small as $1.5 \mathrm{~m} \mathrm{(5 \textrm {ft } )}$ can display significant mixing (Rupert and Plummer, 2004), depending on the magnitude of age gradients over the open interval or the magnitude of pumping. The simplest case of mixing occurs if the water is a binary mixture of old (CFCfree) and young waters; the problem becomes unsolvable if more than two waters mix (Plummer and Busenberg, 1999) because an insufficient number of reliable environmental tracers can be measured in most groundwater samples.

Another approach to groundwater dating assumes a model for age distribution in the system and calculates the response of the system to input of a tracer over time. This approach, using lumped-parameter models (see for example, Małoszewski and Zuber, 1982; Małoszewski and others, 1983; Zuber, 1986; Zuber, 1994; Małoszewski and Zuber, 1996; Cook and Böhlke, 2000), yields estimates of mean residence time of water in the system. The exponential mixing model is perhaps the simplest of the lumped-parameter models and can describe discharge from an unconfined aquifer receiving uniform areal recharge. The exponential model also could apply to discharge from wells with relatively large open intervals that integrate a range of water ages, or water from springs that discharge from a large groundwater reservoir. As multiple lumped-parameter models can be considered, it is usually not possible to determine, on the basis of limited environmental tracer data, which, if any, lumped-parameter model should apply, and model selection is usually based on available geological and other technical information (Małoszewski and Zuber, 1996). When reporting estimates of groundwater age, it is necessary to qualify the age with the model on which it is based. In this study, data were insufficient to justify selection of any lumped-parameter model. Therefore, initial age estimates were based on apparent age assuming piston flow, and in cases in which CFC ratios suggested dilution with old water, a binary mixing model was applied that assumes dilution of a young fraction with old, pretracer water (International Atomic Energy Agency, 2006).

\section{Tritium}

The radioactive isotope of hydrogen, tritium, was used to identify recent groundwater recharge or groundwater mixtures that contain some recent water. In water containing tritium $\left({ }^{3} \mathrm{H}\right),{ }^{3} \mathrm{H}$ substitutes for a hydrogen $\left({ }^{1} \mathrm{H}\right)$ atom or deuterium $\left({ }^{2} \mathrm{H}\right)$ atom in the water molecule and can serve as an excellent tracer because water containing a ${ }^{3} \mathrm{H}$ atom follows the same pathway through the environment as water that does not contain ${ }^{3} \mathrm{H}$ atoms (Plummer and others, 1993, p. 256-257). The half-life of tritium is 12.32 years and commonly is measured in picocuries per liter $(\mathrm{pCi} / \mathrm{L})$ or tritium units $(1 \mathrm{TU}=3.19 \mathrm{pCi} / \mathrm{L})$ (Lucas and Unterweger, 2000).

Low activities of tritium are produced naturally by the interaction of the atmosphere with cosmic rays from the solar wind. Atmospheric thermonuclear weapons testing from 1952 to 1964 introduced a large amount of tritium to the atmosphere that was incorporated directly into water molecules 
Table 1. Locations of air, groundwater, and surface-water sampling sites, Eagle River watershed, 2006-2007, Eagle County, Colorado.

[AIR, air sampling site; GW, groundwater sampling site; SW, surface-water sampling site; map locations using local identification numbers are shown in figure 2]

\begin{tabular}{|c|c|c|c|c|c|}
\hline $\begin{array}{c}\text { Local } \\
\text { identification } \\
\text { number }\end{array}$ & $\begin{array}{l}\text { Site } \\
\text { type }\end{array}$ & $\begin{array}{c}\text { U.S. Geological } \\
\text { Survey station } \\
\text { identification } \\
\text { number }\end{array}$ & $\begin{array}{l}\text { U.S. Geological } \\
\text { Survey } \\
\text { site name }\end{array}$ & $\begin{array}{l}\text { Latitude } \\
\text { (in decimal } \\
\text { degrees) }\end{array}$ & $\begin{array}{l}\text { Longitude } \\
\text { (in decimal } \\
\text { degrees) }\end{array}$ \\
\hline 2 & AIR & 393803106243701 & DONNOVAN PARK & 39.63413889 & -106.41013889 \\
\hline 3 & AIR & 393934106393201 & BELLYACHE RIDGE & 39.65930556 & -106.65888890 \\
\hline 4 & AIR & 393906106494201 & TOWN HALL OF EAGLE & 39.65155556 & -106.82836111 \\
\hline 7 & GW & 393844106195601 & SC00508003CDA & 39.60000000 & -106.30000000 \\
\hline 8 & GW & 393830106210600 & SC00508009AAC & 39.60000000 & -106.30000000 \\
\hline 9 & GW & 393823106215900 & SC00508008BCD-GERALD R FORD PARK & 39.63976389 & -106.36683889 \\
\hline 10 & GW & 393844106232300 & SC00508006DBB-PEDESTRIAN & 39.64572778 & -106.39027222 \\
\hline 11 & GW & 393802106243501 & SC00508112CAB & 39.60000000 & -106.40000000 \\
\hline 12 & GW & 393623106264201 & SC00508122BDD & 39.60655556 & -106.44508333 \\
\hline 17 & GW & 393826106345601 & SC00508204CDB & 39.60000000 & -106.50000000 \\
\hline 18 & GW & 393836106351201 & SC00508204CBC & 39.60000000 & -106.50000000 \\
\hline 19 & GW & 393844106354001 & SC00508205DBA1 & 39.64570556 & -106.59451944 \\
\hline 20 & GW & 393842106354101 & SC00508205DBA2 & 39.64497778 & -106.59467500 \\
\hline 21 & GW & 393748106364201 & SC00508207DAB & 39.62961111 & -106.61247222 \\
\hline 22 & GW & 393812106363901 & SC00508207AAB & 39.63675000 & -106.61102778 \\
\hline 23 & GW & 393823106363801 & SC00508206DDC & 39.63983333 & -106.61061111 \\
\hline 24 & GW & 393905106370801 & SC00508206BAA2 & 39.65156111 & -106.61895833 \\
\hline 25 & GW & 394102106392001 & SC00408323DCD & 39.68396389 & -106.65568889 \\
\hline 26 & GW & 394127106394001 & SC00408323BDD & 39.69102778 & -106.66111111 \\
\hline 27 & GW & 394153106395501 & SC00408314CCC & 39.69794444 & -106.66522222 \\
\hline 28 & GW & 394206106404301 & SC00408315CAD & 39.70179722 & -106.67863889 \\
\hline 37 & GW & 393431106465301 & SC00508435BDB MWB-1 & 39.57513889 & -106.78130556 \\
\hline 38 & GW & 393547106454601 & SC00508424CDC MWB-4 & 39.59630556 & -106.76286111 \\
\hline 39 & GW & 393819106472201 & SC00508410AAB & 39.63858333 & -106.78958333 \\
\hline 40 & GW & 393758106473601 & SC00508410ACD & 39.63280556 & -106.79338889 \\
\hline 41 & GW & 393807106481501 & SC00508410BBD & 39.63522222 & -106.80405556 \\
\hline 42 & GW & 393813106490201 & SC00508409BAD & 39.63686111 & -106.81722222 \\
\hline 43 & GW & 393923106504801 & SC00408431DDB & 39.65641667 & -106.84666667 \\
\hline 44 & GW & 393907106505201 & SC00508406ABA & 39.65188611 & -106.84782222 \\
\hline 45 & GW & 393850106511701 & SC00508406BDD02 & 39.64725278 & -106.85459167 \\
\hline 46 & GW & 393851106511901 & SC00508406BDD01 & 39.64748056 & -106.85530833 \\
\hline 47 & GW & 393917106512501 & SC00408431CBD & 39.65472222 & -106.85697222 \\
\hline 48 & GW & 393904106513601 & SC00508406BBD & 39.65113611 & -106.85987500 \\
\hline 49 & GW & 393847106520401 & SC00508401ACD & 39.64635278 & -106.86768333 \\
\hline 50 & GW & 393903106521901 & SC00508501ABC & 39.65094444 & -106.87183611 \\
\hline 51 & GW & 393900106532901 & SC00508502BAD & 39.65006667 & -106.89146111 \\
\hline 52 & GW & 393702106545401 & SC00508515CBA & 39.61730556 & -106.91505556 \\
\hline 53 & GW & 393740106545801 & SC00508510CBD & 39.62780556 & -106.91608333 \\
\hline 54 & GW & 393819106545901 & SC00508503CDD & 39.63852778 & -106.91655556 \\
\hline 55 & GW & 393832106561101 & SC00508504CBC & 39.64236111 & -106.93630556 \\
\hline 56 & GW & 393831106562701 & SC00508505DDB & 39.64186111 & -106.94088889 \\
\hline 57 & GW & 393845106563401 & SC00508505ADC & 39.64588889 & -106.94272222 \\
\hline
\end{tabular}


Table 1. Locations of air, groundwater, and surface-water sampling sites, Eagle River watershed, 2006-2007, Eagle County, Colorado. - Continued

[AIR, air sampling site; GW, groundwater sampling site; SW, surface-water sampling site; map locations using local identification numbers are shown in figure 2]

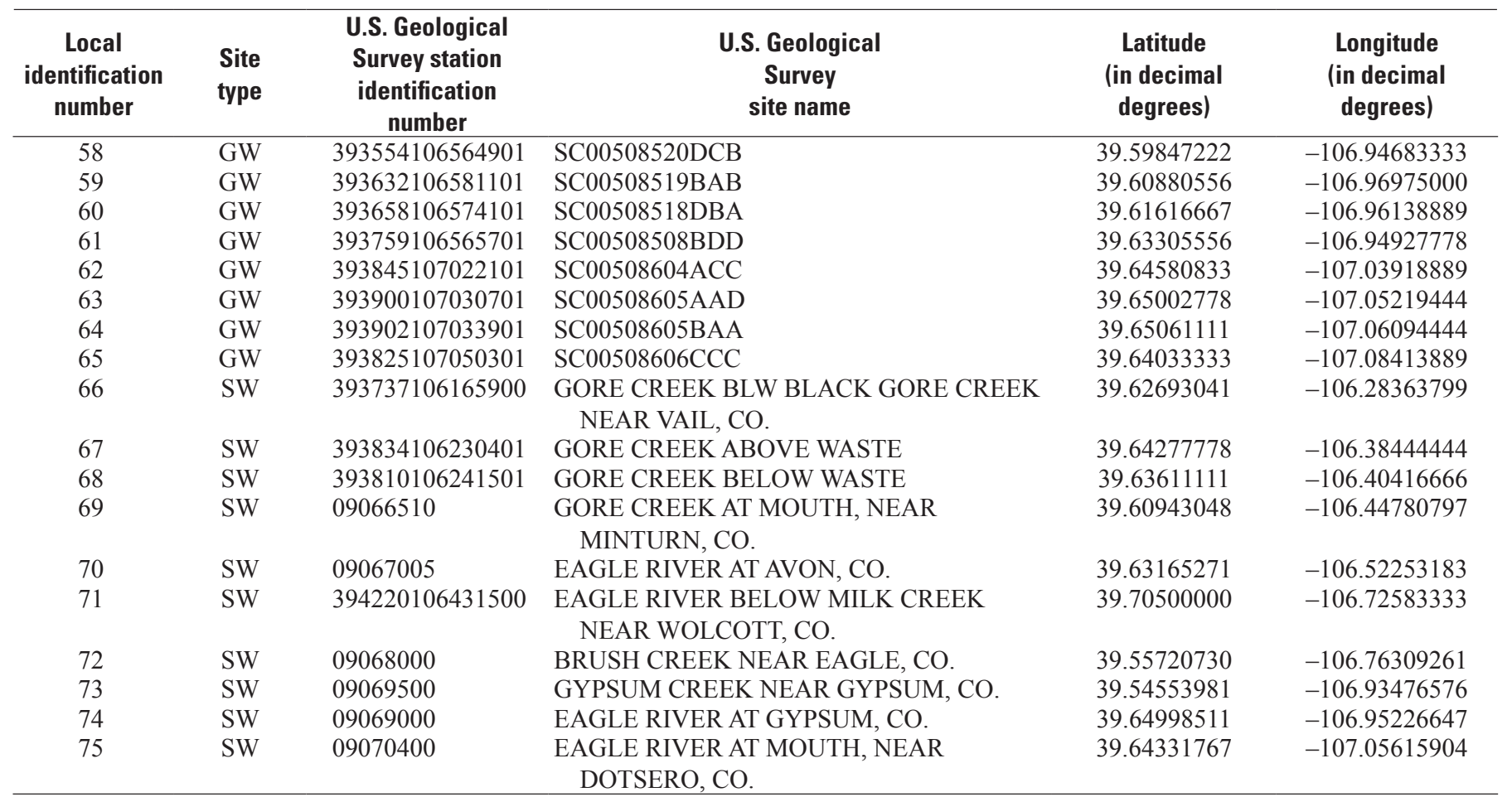

of precipitation (fig. 3). Concentrations in precipitation have been decreasing since that time because of the exchange of water with the oceans, short half-life of tritium, and because atmospheric thermonuclear weapons testing was stopped. Because the concentration of tritium in the atmosphere was high for a relatively short period, its presence in groundwater can identify water that has been recharged during the last 50 years or mixtures that contain a fraction of post-1950s water. Tritium activities in precipitation prior to thermonuclear weapons testing are not well known but probably did not exceed about 6 to $26 \mathrm{pCi} / \mathrm{L}$ (2 to $8 \mathrm{TU}$ ) (Plummer and others, 1993, p. 260). Because tritium has a half-life of 12.32 years, water derived from precipitation before thermonuclear weapons testing would contain a maximum ${ }^{3} \mathrm{H}$ activity of about 0.4 to $1.6 \mathrm{pCi} / \mathrm{L}(0.12$ to 0.5 TU) by the early 2000s. Clark and Fritz (1997, p. 185) state that during the mid-1990s, waters with tritium activities above about $96 \mathrm{pCi} / \mathrm{L}$ (30 TU) contained a considerable component of recharge from the 1960s or the 1970s, and activities above $160 \mathrm{pCi} / \mathrm{L}(50 \mathrm{TU})$ indicate recharge predominantly from the 1960s.

\section{Chlorofluorocarbons}

CFCs were used to estimate apparent ages of recently recharged groundwater and the amounts of dilution of young (post-1940s) groundwater with old (pre-1940s) groundwater. CFCs are stable, synthetic organic compounds that were developed in the early 1930 s as safe alternatives to ammonia and sulfur dioxide in refrigeration and have been used in a wide range of industrial and refrigerant applications (Plummer and Friedman, 1999). Production of CFC-12 (dichlorodifluoromethane) began in 1931, followed by CFC-11 (trichlorofluoromethane) in 1936, and then by many other CFC compounds, most notably CFC-113 (trichlorotrifluoroethane). At ordinary (room) temperatures, $\mathrm{CFC}-113$ is liquid (boiling point $47.6^{\circ} \mathrm{C}\left[118^{\circ} \mathrm{F}\right]$ ), and $\mathrm{CFC}-12$ and $\mathrm{CFC}-11$ are gases (boiling points $-29.8^{\circ} \mathrm{C}\left(-21.6^{\circ} \mathrm{F}\right)$ and $-23.6^{\circ} \mathrm{C}\left(-10.5^{\circ} \mathrm{F}\right)$ respectively). $\mathrm{CFC}-12$ and $\mathrm{CFC}-11$ were used as coolants in air conditioning and refrigeration; as blowing agents in foams, insulation, and packing materials; as propellants in aerosol cans; and as solvents (Plummer and Busenberg, 1999). CFC-113 has been used primarily by the electronics industry in semiconductor chip manufacturing, in vapor degreasing and cold immersion cleaning of microelectronic components, and in surface cleaning. Probably better known as Freon, CFCs are nontoxic, nonflammable, and noncarcinogenic, but they contribute to ozone depletion. Therefore, in 1987, 37 nations signed an agreement to limit release of CFCs and to halve CFC emissions by 2000. This agreement was strengthened in 1990 and 1992 to establish a cutoff date of 1996 to cease production of CFCs in industrialized nations. The United States ceased production on January 1, 1996, as a regulatory requirement under the USEPA Clean Air Act (International Atomic Energy Agency, 2006). 
Table 2. Selected well-construction, major-ion, nutrient, stable isotopes of hydrogen and oxygen, and tritium data from groundwater and surface water, Eagle River watershed, 2006-2007, Eagle County, Colorado.

[--, no data; na, not applicable; GW, groundwater; SW, surface water; m, month; d, day; y, year; >, greater than; <, less than; e, constituent detected, but concentration is estimated; ${ }^{\circ} \mathrm{C}$, degrees Celsius; mg/L, milligrams per liter; $\mu \mathrm{S} / \mathrm{cm}$, microsiemens per centimeter; $\mu \mathrm{g} / \mathrm{L}$, micrograms per liter; $\mathrm{pCi} / \mathrm{L}$, picocuries per liter; ND, not detected; per mil, parts per thousand; pCi/L, picocuries per liter; oxidized, waters under oxidized geochemical conditions; reduced, waters under reducing geochemical conditions; mixture, mixture of waters under oxidized and reduced geochemical conditions]

\begin{tabular}{|c|c|c|c|c|c|c|c|c|c|}
\hline $\begin{array}{c}\text { Local } \\
\text { identification } \\
\text { number }\end{array}$ & $\begin{array}{l}\text { Site } \\
\text { type }\end{array}$ & $\begin{array}{l}\text { U.S. Geological } \\
\text { Survey station } \\
\text { identification number }\end{array}$ & $\begin{array}{c}\text { U.S. Geological } \\
\text { Survey } \\
\text { site name }\end{array}$ & $\begin{array}{l}\text { Latitude } \\
\text { (in decimal } \\
\text { degrees) }\end{array}$ & $\begin{array}{c}\text { Longitude } \\
\text { (in decimal } \\
\text { degrees) }\end{array}$ & $\begin{array}{l}\text { Date of sample } \\
\text { collection } \\
\text { (mmddyy) }\end{array}$ & $\begin{array}{l}\text { Well depth } \\
\text { (feet below } \\
\text { land surface) }\end{array}$ & $\begin{array}{l}\text { Depth to water table } \\
\text { (feet below } \\
\text { land surface) }\end{array}$ & $\begin{array}{c}\text { Specific } \\
\text { conductance, } \\
\text { field }(\mu \mathrm{S} / \mathrm{cm})\end{array}$ \\
\hline 5 & GW & 393743106171000 & SC00508012DCD-BIGHORN PARK & 39.62871944 & -106.2874639 & $06 / 05 / 07$ & 23 & 7.23 & 282 \\
\hline 6 & $\mathrm{GW}$ & 393846106193601 & SC00508003DCA & 39.60000000 & -106.30000000 & $06 / 08 / 07$ & 103 & -- & 314 \\
\hline 7 & GW & 393844106195601 & SC00508003CDA & 39.60000000 & -106.30000000 & $06 / 21 / 07$ & 123 & -- & 329 \\
\hline 8 & GW & 393830106210600 & SC00508009AAC & 39.60000000 & -106.30000000 & $06 / 05 / 07$ & 18 & 2.62 & 280 \\
\hline 9 & GW & 393823106215900 & SC00508008BCD-GERALD R FORD PARK & 39.63976389 & -106.3668389 & $06 / 06 / 07$ & 25 & 13.08 & 451 \\
\hline 10 & GW & 393844106232300 & SC00508006DBB-PEDESTRIAN & 39.64572778 & -106.3902722 & $06 / 06 / 07$ & 41 & 27.12 & 646 \\
\hline 11 & GW & 393802106243501 & SC00508112CAB & 39.60000000 & -106.40000000 & $08 / 24 / 06$ & 50 & -- & 639 \\
\hline 11 & GW & 393802106243501 & SC00508112CAB & 39.60000000 & -106.40000000 & $06 / 19 / 07$ & 50 & -- & 540 \\
\hline 12 & $\mathrm{GW}$ & 393623106264201 & SC00508122BDD & 39.60655556 & -106.4450833 & $06 / 11 / 07$ & 65 & 10.29 & 480 \\
\hline 13 & GW & 393715106280701 & SC00508116BBD & 39.62091667 & -106.4684722 & $06 / 19 / 07$ & 58 & 35.35 & 603 \\
\hline 14 & GW & 393730106301101 & SC00508116BAC & 39.62497222 & -106.5030833 & $06 / 22 / 07$ & 55 & 26.43 & 376 \\
\hline 15 & GW & 393830106340601 & SC00508203CCB & 39.64167778 & -106.5684722 & 06/11/07 & 120 & 72.42 & 588 \\
\hline 16 & GW & 393830106341501 & SC00508204DAA & 39.64127778 & -106.5726111 & $06 / 06 / 07$ & 73 & 39.72 & 490 \\
\hline 17 & $\mathrm{GW}$ & 393826106345601 & SC00508204CDB & 39.60000000 & -106.50000000 & $08 / 24 / 06$ & 80 & -- & 671 \\
\hline 18 & GW & 393836106351201 & SC00508204CBC & 39.60000000 & -106.50000000 & $06 / 08 / 07$ & 61 & -- & 745 \\
\hline 19 & $\mathrm{GW}$ & 393844106354001 & SC00508205DBA1 & 39.64570556 & -106.5945194 & $05 / 21 / 07$ & 115 & 46.51 & 584 \\
\hline 20 & GW & 393842106354101 & SC00508205DBA2 & 39.64497778 & -106.5946750 & $05 / 21 / 07$ & 88 & 53.67 & 911 \\
\hline 21 & GW & 393748106364201 & SC00508207DAB & 39.62961111 & -106.6124722 & $06 / 08 / 07$ & 65 & 11.47 & 468 \\
\hline 22 & GW & 393812106363901 & SC00508207AAB & 39.63675000 & -106.6110278 & $06 / 21 / 07$ & 25 & 14.17 & 530 \\
\hline 23 & $\mathrm{GW}$ & 393823106363801 & SC00508206DDC & 39.63983333 & -106.6106111 & $06 / 11 / 07$ & 36 & 7.69 & 627 \\
\hline 24 & $\mathrm{GW}$ & 393905106370801 & SC00508206BAA2 & 39.65156111 & -106.6189583 & $05 / 21 / 07$ & 50 & -- & 1,690 \\
\hline 25 & $\mathrm{GW}$ & 394102106392001 & SC00408323DCD & 39.68396389 & -106.6556889 & $06 / 09 / 07$ & 63 & 42.74 & 239 \\
\hline 26 & GW & 394127106394001 & SC00408323BDD & 39.69102778 & -106.6611111 & $06 / 09 / 07$ & 34 & 14.27 & 604 \\
\hline 27 & $\mathrm{GW}$ & 394153106395501 & SC00408314CCC & 39.69794444 & -106.6652222 & $06 / 09 / 07$ & 543 & 104.08 & 2,202 \\
\hline 28 & GW & 394206106404301 & SC00408315CAD & 39.70179722 & -106.6786389 & $06 / 07 / 07$ & 46 & 11.66 & 837 \\
\hline 29 & GW & 394243106422501 & SC00408308DDD & 39.71194444 & -106.7069722 & $06 / 07 / 07$ & 20 & 15.35 & 812 \\
\hline 30 & $\mathrm{GW}$ & 394232106424801 & SC00408317ABC & 39.70883333 & -106.7133333 & $05 / 16 / 07$ & 50 & 13.21 & 693 \\
\hline 31 & GW & 394055106452301 & SC00408424DCC & 39.68213889 & -106.7564444 & $05 / 18 / 07$ & 47 & 12.19 & 1,050 \\
\hline 32 & GW & 394040106455701 & SC00408425BCA & 39.67763611 & -106.7658861 & $05 / 15 / 07$ & 65 & 29.72 & 900 \\
\hline 33 & GW & 394036106462101 & SC00408426ADB & 39.67672222 & -106.7725833 & $05 / 15 / 07$ & 62 & -- & 1,021 \\
\hline 34 & GW & 394019106470601 & SC00408426CBD & 39.67203889 & -106.7850444 & $05 / 16 / 07$ & 64 & 25.02 & 1,251 \\
\hline 35 & GW & 394020106475101 & SC00408427CAD1 & 39.67222222 & -106.7974167 & $08 / 25 / 06$ & 71 & 50.80 & 1,030 \\
\hline 36 & GW & 393956106480201 & SC00408434BAC & 39.66547222 & -106.8004722 & $05 / 16 / 07$ & 28 & -- & 2,527 \\
\hline 37 & $\mathrm{GW}$ & 393431106465301 & SC00508435BDB MWB-1 & 39.57513889 & -106.7813056 & $06 / 12 / 07$ & 19 & 10.67 & 449 \\
\hline 38 & $\mathrm{GW}$ & 393547106454601 & SC00508424CDC MWB-4 & 39.59630556 & -106.7628611 & $06 / 12 / 07$ & 17 & 5.58 & 917 \\
\hline 39 & $\mathrm{GW}$ & 393819106472201 & SC00508410AAB & 39.63858333 & -106.7895833 & $06 / 20 / 07$ & 126 & -- & 2,584 \\
\hline 40 & $\mathrm{GW}$ & 393758106473601 & SC00508410ACD & 39.63280556 & -106.7933889 & $06 / 20 / 07$ & 72 & 9.70 & 903 \\
\hline 41 & GW & 393807106481501 & SC00508410BBD & 39.63522222 & -106.8040556 & $06 / 20 / 07$ & 61 & -2.20 & 901 \\
\hline 42 & GW & 393813106490201 & SC00508409BAD & 39.63686111 & -106.8172222 & 06/10/07 & 48 & 9.14 & 778 \\
\hline 43 & GW & 393923106504801 & SC00408431DDB & 39.65641667 & -106.8466667 & $05 / 17 / 07$ & 137 & 118.03 & 1,916 \\
\hline 44 & GW & 393907106505201 & SC00508406ABA & 39.65188611 & -106.8478222 & $05 / 17 / 07$ & 52 & 32.56 & 3,060 \\
\hline 45 & GW & 393850106511701 & SC00508406BDD02 & 39.64725278 & -106.8545917 & $08 / 23 / 06$ & 95 & 19.27 & 2,711 \\
\hline 46 & GW & 393851106511901 & SC00508406BDD01 & 39.64748056 & -106.8553083 & $06 / 10 / 07$ & 46 & 14.24 & 3,490 \\
\hline 47 & GW & 393917106512501 & SC00408431CBD & 39.65472222 & -106.8569722 & $08 / 22 / 06$ & 145 & 126.80 & 5,310 \\
\hline 47 & GW & 393917106512501 & SC00408431CBD & 39.65472222 & -106.8569722 & $06 / 21 / 07$ & 145 & 124.48 & 5,610 \\
\hline 48 & GW & 393904106513601 & SC00508406BBD & 39.65113611 & -106.8598750 & $05 / 23 / 07$ & 48 & 16.29 & 2,042 \\
\hline 49 & $\mathrm{GW}$ & 393847106520401 & SC00508401ACD & 39.64635278 & -106.8676833 & $05 / 17 / 07$ & 380 & 57.33 & 2,630 \\
\hline 50 & $\mathrm{GW}$ & 393903106521901 & SC00508501ABC & 39.65094444 & -106.8718361 & $08 / 22 / 06$ & 55 & 13.69 & 2,517 \\
\hline 51 & $\mathrm{GW}$ & 393900106532901 & SC00508502BAD & 39.65006667 & -106.8914611 & $05 / 20 / 07$ & 60 & 13.90 & 2,780 \\
\hline 52 & GW & 393702106545401 & SC00508515CBA & 39.61730556 & -106.9150556 & $08 / 23 / 06$ & 52 & 19.21 & 1,856 \\
\hline
\end{tabular}


Table 2. Selected well-construction, major-ion, nutrient, stable isotopes of hydrogen and oxygen, and tritium data from groundwater and surface water, Eagle River watershed, 2006-2007, Eagle County, Colorado.-Continued

\begin{tabular}{|c|c|c|c|c|c|c|c|c|c|}
\hline $\begin{array}{c}\text { Local } \\
\text { identification } \\
\text { number }\end{array}$ & $\begin{array}{l}\text { Site } \\
\text { type }\end{array}$ & $\begin{array}{c}\text { U.S. Geological } \\
\text { Survey station } \\
\text { identification number }\end{array}$ & $\begin{array}{c}\text { U.S. Geological } \\
\text { Survey } \\
\text { site name }\end{array}$ & $\begin{array}{l}\text { Latitude } \\
\text { (in decimal } \\
\text { degrees) }\end{array}$ & $\begin{array}{l}\text { Longitude } \\
\text { (in decimal } \\
\text { degrees) }\end{array}$ & $\begin{array}{l}\text { Date of sample } \\
\text { collection } \\
\text { (mmddyy) }\end{array}$ & $\begin{array}{l}\text { Well depth } \\
\text { (feet below } \\
\text { land surface) }\end{array}$ & $\begin{array}{l}\text { Depth to water table } \\
\text { (feet below } \\
\text { land surface) }\end{array}$ & $\begin{array}{c}\text { Specific } \\
\text { conductance, } \\
\text { field }(\mu \mathrm{S} / \mathrm{cm})\end{array}$ \\
\hline 53 & GW & 393740106545801 & SC00508510CBD & 39.62780556 & -106.9160833 & $05 / 22 / 07$ & 100 & 30.81 & 2,075 \\
\hline 54 & GW & 393819106545901 & SC00508503CDD & 39.63852778 & -106.9165556 & $05 / 18 / 07$ & 116 & 40.69 & 2,082 \\
\hline 55 & GW & 393832106561101 & SC00508504CBC & 39.64236111 & -106.9363056 & $05 / 19 / 07$ & 73 & 54.26 & 2,125 \\
\hline 56 & GW & 393831106562701 & SC00508505DDB & 39.64186111 & -106.9408889 & $05 / 19 / 07$ & 50 & 32.51 & 1,890 \\
\hline 57 & GW & 393845106563401 & SC00508505ADC & 39.64588889 & -106.9427222 & $08 / 22 / 06$ & 25 & 6.38 & 1,659 \\
\hline 58 & GW & 393554106564901 & SC00508520DCB & 39.59847222 & -106.9468333 & $05 / 20 / 07$ & 92 & 46.99 & 2,464 \\
\hline 59 & GW & 393632106581101 & SC00508519BAB & 39.60880556 & -106.9697500 & $08 / 23 / 06$ & 89 & 30.03 & 1,144 \\
\hline 60 & GW & 393658106574101 & SC00508518DBA & 39.61616667 & -106.9613889 & $05 / 20 / 07$ & 80 & 3.03 & 594 \\
\hline 61 & GW & 393759106565701 & SC00508508BDD & 39.63305556 & -106.9492778 & $06 / 07 / 07$ & 68 & 47.88 & 1,335 \\
\hline 62 & GW & 393845107022101 & SC00508604ACC & 39.64580833 & -107.0391889 & $08 / 25 / 06$ & 132 & 86.05 & 8,100 \\
\hline 63 & GW & 393900107030701 & SC00508605AAD & 39.65002778 & -107.0521944 & $05 / 19 / 07$ & 48 & 33.24 & 6,530 \\
\hline 64 & GW & 393902107033901 & SC00508605BAA & 39.65061111 & -107.0609444 & $05 / 22 / 07$ & 56 & 26.98 & 2,905 \\
\hline 65 & GW & 393825107050301 & SC00508606CCC & 39.64033333 & -107.0841389 & $05 / 22 / 07$ & 43 & 12.35 & 6,890 \\
\hline 66 & SW & 393737106165900 & $\begin{array}{l}\text { GORE CREEK BLW BLACK GORE CREEK } \\
\text { NEAR VAIL, CO. }\end{array}$ & 39.62693041 & -106.2836380 & $12 / 20 / 06$ & na & na & 223 \\
\hline 66 & SW & 393737106165900 & $\begin{array}{l}\text { GORE CREEK BLW BLACK GORE CREEK } \\
\text { NEAR VAIL, CO. }\end{array}$ & 39.62693041 & -106.2836380 & $03 / 21 / 07$ & na & na & 361 \\
\hline 66 & SW & 393737106165900 & $\begin{array}{l}\text { GORE CREEK BLW BLACK GORE CREEK } \\
\text { NEAR VAIL, CO. }\end{array}$ & 39.62693041 & -106.2836380 & $05 / 23 / 07$ & na & na & 113 \\
\hline 66 & SW & 393737106165900 & $\begin{array}{l}\text { GORE CREEK BLW BLACK GORE CREEK } \\
\text { NEAR VAIL, CO. }\end{array}$ & 39.62693041 & -106.2836380 & $08 / 29 / 07$ & na & na & 176 \\
\hline 69 & SW & 9066510 & $\begin{array}{l}\text { GORE CREEK AT MOUTH, NEAR } \\
\text { MINTURN, CO. }\end{array}$ & 39.60943048 & -106.4478080 & $08 / 24 / 06$ & na & na & 356 \\
\hline 70 & SW & 9067005 & EAGLE RIVER AT AVON, CO. & 39.63165271 & -106.5225318 & $12 / 20 / 06$ & na & na & 313 \\
\hline 70 & SW & 9067005 & EAGLE RIVER AT AVON, CO. & 39.63165271 & -106.5225318 & $03 / 22 / 07$ & na & na & 270 \\
\hline 70 & SW & 9067005 & EAGLE RIVER AT AVON, CO. & 39.63165271 & -106.5225318 & $05 / 23 / 07$ & na & na & 130 \\
\hline 70 & SW & 9067005 & EAGLE RIVER AT AVON, CO. & 39.63165271 & -106.5225318 & $08 / 28 / 07$ & na & na & 248 \\
\hline 71 & SW & 394220106431500 & EAGLE RIVER BELOW MILK CREEK & 39.70500000 & -106.7258333 & $08 / 23 / 06$ & na & na & 762 \\
\hline 71 & SW & 394220106431500 & $\begin{array}{l}\text { EAGLE RIVER BELOW MILK CREEK } \\
\text { NEAR WOLCOTT, CO. }\end{array}$ & 39.70500000 & -106.7258333 & $08 / 25 / 06$ & na & na & 754 \\
\hline 71 & SW & 394220106431500 & $\begin{array}{l}\text { EAGLE RIVER BELOW MILK CREEK } \\
\text { NEAR WOLCOTT, CO. }\end{array}$ & 39.70500000 & -106.7258333 & $12 / 19 / 06$ & na & na & 885 \\
\hline 71 & SW & 394220106431500 & $\begin{array}{l}\text { EAGLE RIVER BELOW MILK CREEK } \\
\text { NEAR WOLCOTT, CO. }\end{array}$ & 39.70500000 & -106.7258333 & $03 / 22 / 07$ & na & na & 560 \\
\hline 71 & SW & 394220106431500 & $\begin{array}{l}\text { EAGLE RIVER BELOW MILK CREEK } \\
\text { NEAR WOLCOTT, CO. }\end{array}$ & 39.70500000 & -106.7258333 & $05 / 22 / 07$ & na & na & 156 \\
\hline 71 & SW & 394220106431500 & $\begin{array}{l}\text { EAGLE RIVER BELOW MILK CREEK } \\
\text { NEAR WOLCOTT, CO. }\end{array}$ & 39.70500000 & -106.7258333 & $08 / 28 / 07$ & na & na & 701 \\
\hline 72 & SW & 9068000 & BRUSH CREEK NEAR EAGLE, CO. & 39.55720730 & -106.7630926 & $12 / 18 / 06$ & na & na & 454 \\
\hline 72 & SW & 9068000 & BRUSH CREEK NEAR EAGLE, CO. & 39.55720730 & -106.7630926 & $03 / 20 / 07$ & na & na & 448 \\
\hline 72 & SW & 9068000 & BRUSH CREEK NEAR EAGLE, CO. & 39.55720730 & -106.7630926 & 05/23/07 & na & na & 225 \\
\hline 72 & SW & 9068000 & BRUSH CREEK NEAR EAGLE, CO. & 39.55720730 & -106.7630926 & 08/30/07 & na & na & 414 \\
\hline 73 & SW & 9069500 & GYPSUM CREEK NEAR GYPSUM, CO. & 39.54553981 & -106.9347658 & $12 / 18 / 06$ & na & na & 445 \\
\hline 73 & SW & 9069500 & GYPSUM CREEK NEAR GYPSUM, CO. & 39.54553981 & -106.9347658 & 03/20/07 & na & na & 490 \\
\hline 73 & SW & 9069500 & GYPSUM CREEK NEAR GYPSUM, CO. & 39.54553981 & -106.9347658 & $05 / 24 / 07$ & na & na & 278 \\
\hline 73 & SW & 9069500 & GYPSUM CREEK NEAR GYPSUM, CO. & 39.54553981 & -106.9347658 & 08/31/07 & na & na & 416 \\
\hline 74 & SW & 9069000 & EAGLE RIVER AT GYPSUM, CO. & 39.64998511 & -106.9522665 & $12 / 18 / 06$ & na & na & 838 \\
\hline 74 & SW & 9069000 & EAGLE RIVER AT GYPSUM, CO. & 39.64998511 & -106.9522665 & $03 / 20 / 07$ & na & na & 742 \\
\hline 74 & SW & 9069000 & EAGLE RIVER AT GYPSUM, CO. & 39.64998511 & -106.9522665 & $05 / 22 / 07$ & na & na & 189 \\
\hline 74 & SW & 9069000 & EAGLE RIVER AT GYPSUM, CO. & 39.64998511 & -106.9522665 & $08 / 27 / 07$ & na & na & 849 \\
\hline
\end{tabular}


Table 2. Selected well-construction, major-ion, nutrient, stable isotopes of hydrogen and oxygen, and tritium data from groundwater and surface water, Eagle River watershed, 2006-2007, Eagle County, Colorado.-Continued

[--, no data; na, not applicable; GW, groundwater; SW, surface water; m, month; d, day; y, year; >, greater than; <, less than; e, constituent detected, but concentration is estimated; ${ }^{\circ} \mathrm{C}, \mathrm{degrees}$ Celsius; $\mathrm{mg} / \mathrm{L}$, milligrams per liter; $\mu \mathrm{S} / \mathrm{cm}$, microsiemens per centimeter; $\mu \mathrm{g} / \mathrm{L}$, micrograms per liter; $\mathrm{pCi} / \mathrm{L}$, picocuries per liter; $\mathrm{ND}$, not detected; per mil, parts per thousand; $\mathrm{pCi} / \mathrm{L}$, picocuries per liter; oxidized, waters under oxidized geochemical conditions; reduced, waters under reducing geochemical conditions; mixture, mixture of waters under oxidized and reduced geochemical conditions]

\begin{tabular}{|c|c|c|c|c|c|c|c|c|c|c|c|c|c|}
\hline $\begin{array}{c}\text { Local } \\
\text { identification } \\
\text { number }\end{array}$ & $\begin{array}{c}\text { pH, water, } \\
\text { whole, field } \\
\text { (standard units) }\end{array}$ & $\begin{array}{c}\text { Temperature, } \\
\text { water } \\
\left({ }^{\circ} \mathrm{C}\right)\end{array}$ & $\begin{array}{c}\text { Oxygen, } \\
\text { dissolved } \\
\text { (mg/L) }\end{array}$ & $\begin{array}{l}\text { Hydrogen sulfide, } \\
\text { unfiltered } \\
\text { (mg/L) }\end{array}$ & $\begin{array}{c}\text { Alkalinity, water, } \\
\text { dissolved, field } \\
\left(\mathrm{mg} / \mathrm{L} \text { as } \mathrm{CaCO}_{3}\right)\end{array}$ & $\begin{array}{c}\text { Total } \\
\text { dissolved } \\
\text { solids } \\
\text { (mg/L) }\end{array}$ & $\begin{array}{l}\text { Calcium, } \\
\text { dissolved } \\
\text { (mg/L) }\end{array}$ & $\begin{array}{l}\text { Magnesium, } \\
\text { dissolved } \\
\text { (mg/L) }\end{array}$ & $\begin{array}{l}\text { Sodium, } \\
\text { dissolved } \\
\text { (mg/L) }\end{array}$ & $\begin{array}{c}\text { Potassium, } \\
\text { dissolved } \\
\text { (mg/L) }\end{array}$ & $\begin{array}{c}\text { Bicarbonate, } \\
\text { water, } \\
\text { dissolved, field, } \\
\text { (mg/L as } \mathrm{HCO}_{3} \text { ) }\end{array}$ & $\begin{array}{c}\text { Sulfate, } \\
\text { dissolved } \\
\text { (mg/L) }\end{array}$ & $\begin{array}{c}\text { Chloride, } \\
\text { dissolved } \\
\text { (mg/L) }\end{array}$ \\
\hline 5 & 7.1 & 12.4 & 3.5 & $<0.1$ & 136 & e 155 & 48.5 & 3.93 & 4.46 & 1.20 & 166 & 4.27 & 3.30 \\
\hline 6 & 7.9 & 7.0 & 4.0 & $<.1$ & 115 & e 165 & 49.9 & 4.93 & 5.89 & 1.25 & 140 & 5.25 & 20.9 \\
\hline 7 & 7.9 & 7.0 & 3.6 & $<.1$ & 122 & e 172 & 47.9 & 5.57 & 7.42 & 1.20 & 149 & 5.34 & 23.1 \\
\hline 8 & 7.8 & 8.0 & 2.2 & $<.1$ & 115 & 148 & 39.5 & 6.11 & 5.54 & 1.30 & 140 & 5.07 & 13.1 \\
\hline 9 & 7.8 & 3.9 & 4.2 & $<.1$ & 186 & e 238 & 67.9 & 9.41 & 8.80 & 1.05 & 227 & 8.26 & 21.2 \\
\hline 10 & 7.3 & 9.0 & 3.9 & $<.1$ & 277 & e 351 & 105 & 16.0 & 7.72 & 1.76 & 338 & 26.3 & 15.1 \\
\hline 11 & 7.3 & 9.0 & 2.4 & $<.1$ & 177 & 373 & 91.3 & 18.2 & 9.25 & 1.53 & -- & 101 & 33.6 \\
\hline 11 & 7.5 & 7.4 & 3.0 & $<.1$ & 147 & 298 & 74.9 & 14.6 & 8.14 & 1.29 & 180 & 73.1 & 27.8 \\
\hline 12 & 7.7 & 8.0 & 2.9 & $<.1$ & 179 & e 269 & 60.3 & 19.1 & 9.00 & 1.51 & 218 & 42.9 & 14.2 \\
\hline 13 & 7.4 & 9.6 & 4.6 & $<.1$ & 168 & e 345 & 83.3 & 19.6 & 9.59 & 1.40 & 205 & 86.1 & 30.7 \\
\hline 14 & 7.3 & 9.1 & 1.7 & $<.1$ & 132 & e 206 & 53.2 & 10.0 & 5.87 & 1.09 & 162 & 26.4 & 17.4 \\
\hline 15 & 7.6 & 9.9 & 5.8 & $<.1$ & 222 & e 370 & 72.3 & 22.3 & 21.6 & 1.59 & 271 & 83.5 & 9.68 \\
\hline 16 & 7.3 & 10.2 & 2.0 & $<.1$ & 230 & e 272 & 79.9 & 13.7 & 4.88 & 1.65 & 281 & 19.8 & 4.59 \\
\hline 17 & 7.3 & 9.4 & 3.4 & $<.1$ & 197 & e 410 & 95.6 & 23.8 & 6.85 & 1.81 & -- & 127 & 18.4 \\
\hline 18 & 7.6 & 9.4 & 4.0 & $<.1$ & 197 & e 466 & 113 & 25.6 & 7.74 & 1.98 & 240 & 162 & 19.6 \\
\hline 19 & 7.7 & 9.4 & 3.1 & $<.1$ & 163 & 337 & 81.3 & 18.9 & 8.16 & 1.55 & 198 & 83.9 & 28.1 \\
\hline 20 & 7.5 & 10.4 & 4.4 & $<.1$ & 204 & e 510 & 118 & 29.0 & 18.4 & 2.14 & 249 & 94.5 & 101 \\
\hline 21 & 7.7 & 9.3 & 4.8 & $<.1$ & 144 & e 281 & 77.8 & 8.88 & 5.68 & 1.51 & 175 & 80.5 & 6.11 \\
\hline 22 & 7.6 & 9.6 & 5.4 & $<.1$ & 145 & 332 & 90.0 & 11.5 & 3.98 & 1.39 & 176 & 123 & 3.59 \\
\hline 23 & 7.5 & 9.1 & 5.7 & $<.1$ & 175 & e 390 & 106 & 13.6 & 3.90 & 1.53 & 213 & 143 & 4.19 \\
\hline 24 & 7.6 & 8.4 & 7.5 & $<.1$ & 199 & e 1,040 & 137 & 34.7 & 159 & 3.66 & 243 & 384 & 192 \\
\hline 25 & 7.5 & 9.9 & 6.0 & $<.1$ & 63 & 137 & 20.6 & 5.56 & 19.2 & 1.00 & 77 & 30.8 & 13.4 \\
\hline 26 & 7.8 & 10.2 & 1.0 & .1 & 197 & 341 & 67.7 & 18.0 & 31.3 & 4.82 & 240 & 38.1 & 48.9 \\
\hline 27 & 8.6 & 16.2 & 0.1 & .2 & 421 & 1,260 & 3.84 & 0.72 & 510 & 0.53 & 513 & 75.0 & 402 \\
\hline 28 & 7.5 & 11.1 & 7.2 & $<.1$ & 251 & 488 & 91.8 & 19.9 & 61.3 & 2.34 & 306 & 79.4 & 70.3 \\
\hline 29 & 7.5 & 8.4 & 3.0 & $<.1$ & 191 & e 484 & 80.5 & 20.3 & 61.0 & 2.40 & 233 & 99.3 & 85.9 \\
\hline 30 & 7.7 & 9.4 & 4.3 & $<.1$ & 247 & e 424 & 72.7 & 18.8 & 41.8 & 2.30 & 301 & 65.7 & 58.9 \\
\hline 31 & 7.6 & 10.7 & 1.9 & $<.1$ & 170 & e 667 & 133 & 24.6 & 58.1 & 2.68 & 207 & 270 & 62.2 \\
\hline 32 & 7.7 & 11.2 & 3.2 & $<.1$ & 224 & 532 & 110 & 24.0 & 44.8 & 3.28 & 274 & 140 & 53.6 \\
\hline 33 & 7.7 & 10.9 & 6.5 & $<.1$ & 231 & 587 & 106 & 31.7 & 50.4 & 11.1 & 281 & 125 & 98.2 \\
\hline 34 & 7.6 & 11.7 & 2.8 & $<.1$ & 198 & 823 & 186 & 27.3 & 47.4 & 3.60 & 242 & 349 & 75.4 \\
\hline 35 & 7.2 & 13.0 & 4.5 & $<.1$ & 273 & e 627 & 140 & 16.7 & 46.5 & 3.02 & -- & 175 & 64.4 \\
\hline 36 & 7.1 & 10.6 & 0.4 & $<.1$ & 328 & e 2,270 & 585 & 43.6 & 84.7 & 5.92 & 399 & 1,164 & 171 \\
\hline 37 & 7.3 & 7.5 & 3.8 & $<.1$ & 241 & e 253 & 65.9 & 17.4 & 3.82 & 1.11 & 293 & 4.39 & 1.08 \\
\hline 38 & 7.0 & 8.0 & 0.6 & $<.1$ & 277 & e 602 & 162 & 20.7 & 7.03 & 1.38 & 338 & 229 & 2.90 \\
\hline 39 & 7.1 & 12.4 & 4.6 & $<.1$ & 235 & e 2,190 & 476 & 99.1 & 36.1 & 11.1 & 286 & 1,368 & 42.5 \\
\hline 40 & 7.4 & 10.9 & 5.6 & $<.1$ & 195 & 541 & 111 & 19.9 & 43.1 & 2.85 & 238 & 177 & 55.2 \\
\hline 41 & 7.3 & 10.9 & 6.2 & $<.1$ & 201 & e 535 & 117 & 20.5 & 33.1 & 2.93 & 245 & 170 & 55.5 \\
\hline 42 & 7.4 & 9.4 & 4.7 & $<.1$ & 163 & 475 & 105 & 16.7 & 29.0 & 2.94 & 198 & 158 & 51.6 \\
\hline 43 & 7.3 & 10.5 & 8.6 & $<.1$ & 181 & e 1,580 & 366 & 65.3 & 27.3 & 3.36 & 221 & 977 & 10.3 \\
\hline 44 & 7.2 & 11.1 & 6.3 & $<.1$ & 206 & e 2,670 & 454 & 184 & 118 & 4.60 & 251 & 1,672 & 83.2 \\
\hline 45 & 7.3 & 10.3 & 0.1 & $<.1$ & 194 & 2,260 & 388 & 153 & 56.6 & 7.59 & -- & 1,458 & 71.0 \\
\hline 46 & 7.0 & 10.1 & 0.8 & $<.1$ & 275 & 2,810 & 613 & 105 & 104 & 33.5 & 335 & 1,530 & 240 \\
\hline
\end{tabular}


Table 2. Selected well-construction, major-ion, nutrient, stable isotopes of hydrogen and oxygen, and tritium data from groundwater and surface water, Eagle River watershed, 2006-2007, Eagle County, Colorado.-Continued

\begin{tabular}{|c|c|c|c|c|c|c|c|c|c|c|c|c|c|}
\hline $\begin{array}{c}\text { Local } \\
\text { identification } \\
\text { number }\end{array}$ & $\begin{array}{c}\text { pH, water, } \\
\text { whole, field } \\
\text { (standard units) }\end{array}$ & $\begin{array}{c}\text { Temperature, } \\
\text { water } \\
\left({ }^{\circ} \mathrm{C}\right)\end{array}$ & $\begin{array}{c}\text { Oxygen, } \\
\text { dissolved } \\
\text { (mg/L) }\end{array}$ & $\begin{array}{l}\text { Hydrogen sulfide, } \\
\text { unfiltered } \\
\text { (mg/L) }\end{array}$ & $\begin{array}{c}\text { Alkalinity, water, } \\
\text { dissolved, field } \\
\left.\text { (mg/L as } \mathrm{CaCO}_{3}\right)\end{array}$ & $\begin{array}{c}\text { Total } \\
\text { dissolved } \\
\text { solids } \\
\text { (mg/L) }\end{array}$ & $\begin{array}{c}\text { Calcium, } \\
\text { dissolved } \\
\text { (mg/L) }\end{array}$ & $\begin{array}{l}\text { Magnesium, } \\
\text { dissolved } \\
(\mathrm{mg} / \mathrm{L})\end{array}$ & $\begin{array}{l}\text { Sodium, } \\
\text { dissolved } \\
\text { (mg/L) }\end{array}$ & $\begin{array}{l}\text { Potassium, } \\
\text { dissolved } \\
\text { (mg/L) }\end{array}$ & $\begin{array}{c}\text { Bicarbonate, } \\
\text { water, } \\
\text { dissolved, field, } \\
\text { (mg/L as } \mathrm{HCO}_{3} \text { ) }\end{array}$ & $\begin{array}{c}\text { Sulfate, } \\
\text { dissolved } \\
\text { (mg/L) }\end{array}$ & $\begin{array}{c}\text { Chloride, } \\
\text { dissolved } \\
\text { (mg/L) }\end{array}$ \\
\hline 47 & 7.2 & 13.6 & 3.7 & $<.1$ & 102 & e 4,690 & 606 & 321 & 332 & 7.34 & -- & 2,396 & 925 \\
\hline 47 & 7.4 & 12.9 & 4.1 & $<.1$ & 98 & e 4,550 & 616 & 318 & 370 & 7.04 & 120 & 2,355 & 785 \\
\hline 48 & 7.2 & 11.8 & 6.4 & $<.1$ & 177 & 1,750 & 485 & 18.9 & 27.7 & 3.01 & 216 & 1,057 & 37.2 \\
\hline 49 & 7.1 & 11.2 & 6.6 & $<.1$ & 267 & e 2,380 & 621 & 65.5 & 36.3 & 5.91 & 325 & 1,425 & 42.8 \\
\hline 50 & 7.1 & 12.8 & 6.1 & $<.1$ & 174 & e 2,120 & 544 & 21.7 & 21.6 & 4.49 & -- & 1,369 & 22.0 \\
\hline 51 & 7.2 & 10.6 & 1.3 & $<.1$ & 218 & 2,070 & 457 & 59.1 & 127 & 2.37 & 266 & 1,056 & 229 \\
\hline 52 & 7.1 & 9.6 & 6.7 & $<.1$ & 214 & e 1,440 & 306 & 73.3 & 23.9 & 2.80 & -- & 870 & 17.3 \\
\hline 53 & 7.2 & 9.6 & 0.2 & .1 & 195 & 1,750 & 459 & 48.2 & 16.7 & 2.97 & 238 & 1,076 & 17.0 \\
\hline 54 & 7.4 & 11.4 & 6.0 & $<.1$ & 235 & e 1,740 & 350 & 78.6 & 57.3 & 2.41 & 286 & 1,046 & 42.2 \\
\hline 55 & 7.1 & 11.4 & 5.5 & $<.1$ & 216 & e 1,830 & 465 & 55.6 & 8.65 & 3.61 & 264 & 1,132 & 7.20 \\
\hline 56 & 7.1 & 11.1 & 4.5 & $<.1$ & 307 & 1,610 & 423 & 40.8 & 7.26 & 3.95 & 375 & 921 & 2.36 \\
\hline 57 & 7.0 & 12.5 & 4.2 & $<.1$ & 240 & 1,240 & 309 & 39.8 & 7.28 & 3.42 & -- & 712 & 2.67 \\
\hline 58 & 7.2 & 11.2 & 4.1 & $<.1$ & 224 & e 2,090 & 461 & 83.1 & 51.6 & 2.61 & 274 & 1,310 & 24.6 \\
\hline 59 & 7.4 & 13.5 & 6.2 & $<.1$ & -- & e 682 & 67.6 & 29.6 & 118 & 2.70 & -- & 253 & 67.1 \\
\hline 60 & 7.5 & 8.5 & 3.9 & $<.1$ & 199 & 363 & 88.0 & 22.5 & 4.45 & 1.22 & 243 & 111 & 1.37 \\
\hline 61 & 7.4 & 11.6 & 4.8 & $<.1$ & 273 & e 1,040 & 256 & 44.8 & 6.73 & 2.45 & 333 & 539 & 2.69 \\
\hline 62 & 7.4 & 15.1 & 0.1 & $<.1$ & 188 & 5,320 & 471 & 109 & 1,217 & 12.9 & -- & 1,380 & 2,000 \\
\hline 63 & 7.2 & 12.4 & 0.6 & $<.1$ & 289 & e 4,540 & 534 & 156 & 756 & 23.3 & 353 & 1,426 & 1,445 \\
\hline 64 & 7.4 & 11.0 & 0.3 & $<.1$ & 255 & 2,400 & 425 & 69.2 & 223 & 12.1 & 311 & 1,375 & 126 \\
\hline 65 & 7.5 & 11.7 & 0.1 & .2 & 362 & 4,860 & 596 & 280 & 628 & 10.1 & 441 & 1,665 & 1,428 \\
\hline 66 & 8.3 & 0.2 & 10.4 & -- & 66 & e 115 & 23.7 & 4.65 & 11.9 & .59 & 80 & 3.31 & 25.6 \\
\hline 66 & 8.4 & 1.5 & 10.8 & -- & 56 & e 178 & 26.9 & 5.72 & 32.7 & .77 & 68 & 3.24 & 69.5 \\
\hline 66 & 8.1 & 3.5 & 9.8 & -- & 43 & e 60 & 14.4 & 2.55 & 4.10 & .44 & 51 & 1.43 & 7.09 \\
\hline 66 & 8.5 & 12.6 & 7.9 & -- & 62 & 93 & 22.3 & 4.58 & 7.03 & .67 & 75 & 3.38 & 12.8 \\
\hline 69 & 9.0 & 13.2 & 8.8 & -- & 117 & e 205 & 52.5 & 9.06 & 7.52 & 1.38 & 130 & 42.4 & 14.8 \\
\hline 70 & 8.2 & 0.2 & 11.0 & -- & 85 & e 180 & 38.8 & 11.5 & 6.89 & 1.10 & 103 & 53.2 & 8.76 \\
\hline 70 & 8.2 & 2.0 & 11.0 & -- & 72 & e 151 & 31.4 & 8.80 & 8.90 & 1.04 & 87 & 35.1 & 14.6 \\
\hline 70 & 8.2 & 3.8 & 10.3 & -- & 51 & e 72 & 17.3 & 4.50 & 2.24 & .55 & 62 & 8.43 & 2.40 \\
\hline 70 & 8.6 & 16.3 & 8.1 & -- & 76 & e 139 & 32.2 & 8.20 & 3.71 & .97 & 91 & 36.7 & 5.61 \\
\hline 71 & 9.0 & 21.2 & 6.8 & -- & 109 & 424 & 60.0 & 14.1 & 72.0 & 2.72 & 124 & 92.7 & 109 \\
\hline 71 & -- & 18.0 & -- & -- & -- & -- & -- & -- & -- & -- & -- & -- & -- \\
\hline 71 & 8.6 & 0.0 & 11.8 & -- & 106 & 492 & 61.7 & 15.2 & 85.7 & 2.84 & 127 & 108 & 141 \\
\hline 71 & 8.7 & 6.7 & 10.4 & -- & 87 & e 315 & 44.7 & 12.1 & 46.8 & 2.00 & 104 & 73.4 & 74.7 \\
\hline 71 & 8.2 & 5.5 & 10.0 & -- & 48 & e 84 & 18.1 & 4.50 & 5.07 & .63 & 58 & 14.7 & 6.08 \\
\hline 71 & 8.5 & 15.2 & 11.3 & -- & 96 & e 388 & 55.7 & 12.4 & 68.6 & 2.56 & 114 & 82.5 & 102 \\
\hline 72 & 8.2 & 1.7 & 10.6 & -- & 106 & e 287 & 68.4 & 13.9 & 3.09 & .67 & 128 & 127 & 0.86 \\
\hline 72 & 8.5 & 8.3 & 9.3 & -- & 82 & e 273 & 69.8 & 13.6 & 3.12 & .71 & 98 & 126 & 1.10 \\
\hline 72 & 8.3 & 3.9 & 10.5 & -- & 70 & 130 & 34.0 & 6.30 & 1.63 & .47 & 85 & 38.0 & 0.56 \\
\hline 72 & 8.5 & 15.0 & 7.8 & -- & 106 & e 263 & 66.8 & 13.1 & 2.97 & .67 & 126 & 106 & 0.86 \\
\hline 73 & 8.3 & 1.1 & 11.0 & -- & 114 & e 288 & 79.9 & 9.30 & 2.45 & .46 & 137 & 118 & 0.46 \\
\hline 73 & 8.5 & 5.8 & 9.9 & -- & 115 & e 321 & 86.8 & 10.5 & 2.77 & .55 & 137 & 142 & 0.60 \\
\hline 73 & 8.4 & 8.0 & 9.3 & -- & 86 & e 170 & 46.7 & 5.73 & 1.75 & .34 & 103 & 56.0 & 0.33 \\
\hline 73 & 8.4 & 10.1 & 8.7 & -- & 113 & e 259 & 72.7 & 8.33 & 2.31 & .40 & 135 & 98.1 & 0.38 \\
\hline 74 & 8.6 & 0.9 & 12.8 & -- & 118 & e 501 & 83.5 & 17.5 & 61.3 & 2.57 & 139 & 155 & 98.8 \\
\hline 74 & 8.2 & 7.4 & 9.9 & -- & 113 & 436 & 70.4 & 16.2 & 55.6 & 2.67 & 136 & 126 & 86.2 \\
\hline 74 & 8.1 & 6.3 & 10.0 & -- & 54 & e 105 & 23.3 & 4.97 & 5.66 & .74 & 66 & 25.0 & 6.63 \\
\hline 74 & 8.6 & 16.5 & 10.7 & -- & 125 & 511 & 95.5 & 16.4 & 57.8 & 2.91 & 147 & 172 & 85.0 \\
\hline
\end{tabular}


Table 2. Selected well-construction, major-ion, nutrient, stable isotopes of hydrogen and oxygen, and tritium data from groundwater and surface water, Eagle River watershed, 2006-2007, Eagle County, Colorado.-Continued

[--, no data; na, not applicable; GW, groundwater; SW, surface water; $\mathrm{m}$, month; $\mathrm{d}$, day; y, year; >, greater than; <, less than; e, constituent detected, but concentration is estimated; ${ }^{\circ} \mathrm{C}$, degrees Celsius; $\mathrm{mg} / \mathrm{L}$, milligrams per liter; $\mu \mathrm{S} / \mathrm{cm}$, microsiemens per centimeter; $\mu \mathrm{g} / \mathrm{L}$, micrograms per liter; $\mathrm{pCi} / \mathrm{L}$, picocuries per liter; $\mathrm{ND}$, not detected; per mil, parts per thousand; $\mathrm{pCi} / \mathrm{L}$, picocuries per liter; oxidized, waters under oxidized geochemical conditions; reduced, waters under reducing geochemical conditions; mixture, mixture of waters under oxidized and reduced geochemical conditions]

\begin{tabular}{|c|c|c|c|c|c|c|c|c|c|c|c|c|c|c|}
\hline $\begin{array}{c}\text { Local } \\
\text { identification } \\
\text { number }\end{array}$ & $\begin{array}{l}\text { Fluoride, } \\
\text { dissolved } \\
\text { (mg/L) }\end{array}$ & $\begin{array}{c}\text { Silica, } \\
\text { dissolved } \\
\text { (mg/L) }\end{array}$ & $\begin{array}{c}\text { Nitrite, } \\
\text { dissolved } \\
\text { (mg/L) }\end{array}$ & $\begin{array}{l}\text { Nitrite plus } \\
\text { nitrate, dissolved, } \\
\text { as nitrogen } \\
\text { (mg/L) }\end{array}$ & $\begin{array}{l}\text { Ammonia, } \\
\text { dissolved } \\
\text { (mg/L) }\end{array}$ & $\begin{array}{l}\text { Orthophosphate, } \\
\text { dissolved } \\
\text { (mg/L) }\end{array}$ & $\begin{array}{c}\text { Boron, } \\
\text { dissolved } \\
(\mu \mathrm{g} / \mathrm{L})\end{array}$ & $\begin{array}{c}\text { Iron, } \\
\text { dissolved } \\
(\mu \mathrm{g} / \mathrm{L})\end{array}$ & $\begin{array}{l}\text { Manganese, } \\
\text { dissolved } \\
(\mu \mathrm{g} / \mathrm{L})\end{array}$ & $\begin{array}{l}\text { Oxidation } \\
\text { state }\end{array}$ & $\begin{array}{l}\text { Hydrogen } \\
2 / 1 \text { ratio } \\
\text { (per mil) }\end{array}$ & $\begin{array}{l}\text { Oxygen } \\
\text { 18/16 ratio } \\
\text { (per mil) }\end{array}$ & $\begin{array}{l}\text { Tritium } \\
\text { (pCi/L) }\end{array}$ & $\begin{array}{l}\text { Tritium, } 2 \text { sigma } \\
\text { precision } \\
\text { estimate } \\
\text { (pCi/L) }\end{array}$ \\
\hline 5 & e 0.05 & 5.42 & e 0.001 & 0.37 & $<0.02$ & e 0.004 & 5 & 60 & 13.0 & oxidized & -126 & -17.34 & 27.5 & 0.96 \\
\hline 6 & e .07 & 6.62 & $<.002$ & .37 & $<.02$ & e .003 & 6 & $<6$ & $<0.2$ & oxidized & -129 & -17.32 & 24.6 & .96 \\
\hline 7 & e .09 & 6.25 & $<.002$ & .37 & $<.02$ & e .004 & 7 & $<6$ & $<.2$ & oxidized & -127 & -17.28 & 32.3 & .96 \\
\hline 8 & .12 & 6.77 & $<.002$ & .23 & $<.02$ & $<.006$ & 7 & $<6$ & 12.2 & oxidized & -126 & -17.23 & 28.2 & .96 \\
\hline 9 & e .06 & 6.21 & $<.002$ & .79 & $<.02$ & e .004 & 7 & $<6$ & $<.2$ & oxidized & -126 & -16.99 & 29.1 & .96 \\
\hline 10 & e.06 & 10.6 & $<.002$ & .70 & $<.02$ & e .005 & 15 & e 4 & e. 1 & oxidized & -125 & -16.75 & 21.4 & .74 \\
\hline 11 & .13 & 7.81 & $<.002$ & .99 & .01 & .011 & 26 & 7 & $<.6$ & oxidized & -129 & -17.33 & 33.9 & 1.31 \\
\hline 11 & .12 & 6.95 & $<.002$ & .62 & $<.02$ & .011 & 24 & e 4 & e. 2 & oxidized & -127 & -17.40 & 27.8 & .96 \\
\hline 12 & e .07 & 9.62 & $<.002$ & 1.10 & $<.02$ & .007 & 42 & $<6$ & e. 1 & oxidized & -128 & -17.18 & 26.2 & .96 \\
\hline 13 & e .09 & 8.12 & $<.002$ & 1.30 & $<.02$ & e .004 & 21 & 20 & .6 & oxidized & -128 & -17.22 & 27.8 & .96 \\
\hline 14 & e .07 & 8.59 & $<.002$ & .79 & $<.02$ & e .004 & 12 & 20 & .6 & oxidized & -127 & -16.99 & 20.2 & .96 \\
\hline 15 & e .08 & 13.2 & $<.002$ & 2.71 & $<.02$ & .010 & 37 & e 4 & e. 1 & oxidized & -130 & -17.50 & 45.8 & 1.28 \\
\hline 16 & e .08 & 7.43 & $<.002$ & .48 & $<.02$ & e .005 & 13 & $<6$ & e.1 & oxidized & -126 & -17.16 & 31.4 & .96 \\
\hline 17 & .15 & 9.16 & $<.002$ & 1.94 & e .01 & e .005 & 25 & $<6$ & $<.6$ & oxidized & -126 & -17.04 & 29.0 & .96 \\
\hline 18 & .11 & 9.47 & $<.002$ & 2.11 & $<.02$ & e .005 & 28 & 7 & e .2 & oxidized & -128 & -17.00 & 29.4 & .96 \\
\hline 19 & .12 & 9.37 & $<.002$ & 1.80 & $<.02$ & $<.006$ & 23 & 25 & 2.1 & oxidized & -126 & -16.96 & 31.4 & .96 \\
\hline 20 & .14 & 11.9 & $<.002$ & 2.74 & $<.02$ & e .005 & 34 & $<6$ & $<.2$ & oxidized & -121 & -16.28 & 32.6 & .96 \\
\hline 21 & .23 & 11.2 & $<.002$ & .57 & $<.02$ & e .005 & 15 & 7 & .2 & oxidized & -117 & -16.25 & 26.6 & .96 \\
\hline 22 & .18 & 9.15 & $<.002$ & .61 & $<.02$ & .007 & 11 & 21 & .4 & oxidized & -120 & -16.42 & 30.7 & 1.28 \\
\hline 23 & .20 & 10.3 & $<.002$ & .44 & $<.02$ & e .006 & 14 & 9 & .5 & oxidized & -119 & -16.32 & 31.7 & 1.28 \\
\hline 24 & .10 & 9.26 & $<.002$ & .45 & $<.02$ & e .003 & 46 & 10 & 22.9 & oxidized & -127 & -17.11 & 25.6 & .96 \\
\hline 25 & .22 & 6.26 & $<.002$ & .39 & $<.02$ & .066 & 10 & 29 & 1.8 & oxidized & -128 & -17.33 & 31.7 & 1.28 \\
\hline 26 & .14 & 11.3 & .006 & .15 & .59 & .031 & 21 & 550 & 631.6 & mixture & -121 & -16.16 & 30.4 & 1.28 \\
\hline 27 & 5.41 & 8.34 & $<.002$ & $<.06$ & .18 & .030 & 161 & 18 & 5.5 & reduced & -131 & -18.05 & 0.45 & .51 \\
\hline 28 & .24 & 11.8 & $<.002$ & $<.06$ & .00 & .018 & 35 & $<6$ & 98.3 & mixture & -125 & -16.69 & 24.0 & .96 \\
\hline 29 & .14 & 9.78 & $<.002$ & 2.25 & $<.02$ & .007 & 34 & $<6$ & e. 1 & oxidized & -126 & -16.96 & 25.0 & .96 \\
\hline 30 & .13 & 12.1 & $<.002$ & .87 & $<.02$ & .011 & 31 & $<6$ & e. 2 & oxidized & -128 & -17.18 & 30.4 & 1.28 \\
\hline 31 & .13 & 10.7 & $<.002$ & .71 & $<.02$ & e .004 & 40 & 25 & 1.9 & oxidized & -125 & -16.96 & 24.3 & .96 \\
\hline 32 & .17 & 15.1 & $<.002$ & 1.43 & $<.02$ & .007 & 39 & 8 & .5 & oxidized & -126 & -16.92 & 25.9 & .96 \\
\hline 33 & .12 & 11.7 & $<.002$ & 3.25 & $<.02$ & .007 & 40 & 25 & 1.2 & oxidized & -123 & -16.63 & 27.5 & .96 \\
\hline 34 & .19 & 12.5 & $<.002$ & .64 & $<.02$ & .006 & 41 & 8 & .6 & oxidized & -125 & -16.94 & 22.4 & .74 \\
\hline 35 & .25 & 12.7 & $<.002$ & 1.02 & e .01 & .007 & 32 & e 6 & e .4 & oxidized & -125 & -17.00 & 28.0 & .90 \\
\hline 36 & .25 & 13.0 & $<.002$ & .78 & $<.02$ & e .004 & 76 & 203 & 5.0 & mixture & -125 & -16.80 & 29.4 & .96 \\
\hline 37 & e .08 & 14.4 & $<.002$ & e .05 & $<.02$ & .028 & 16 & 11 & 6.3 & oxidized & -131 & -17.37 & 22.7 & .96 \\
\hline 38 & .12 & 13.0 & $<.002$ & e. 04 & $<.02$ & .006 & 29 & 35 & 2.1 & oxidized & -126 & -16.93 & 25.0 & .96 \\
\hline 39 & .40 & 14.7 & $<.002$ & .26 & $<.02$ & e .004 & 65 & 25 & 14.6 & oxidized & -126 & -17.14 & 31.0 & .96 \\
\hline 40 & .13 & 12.9 & $<.002$ & .36 & $<.02$ & .007 & 30 & 13 & 2.4 & oxidized & -126 & -17.03 & 25.6 & .96 \\
\hline 41 & .14 & 13.6 & $<.002$ & .41 & $<.02$ & .009 & 28 & e 5 & .6 & oxidized & -126 & -17.14 & 22.4 & .96 \\
\hline 42 & .13 & 11.6 & $<.002$ & .35 & $<.02$ & .008 & 20 & 151 & 4.3 & mixture & -126 & -17.17 & 26.9 & .96 \\
\hline 43 & .31 & 16.8 & $<.002$ & 1.19 & $<.02$ & e .006 & 122 & 102 & 3.0 & mixture & -123 & -16.32 & 32.0 & .96 \\
\hline 44 & .31 & 16.7 & .003 & 3.88 & e .02 & .008 & 332 & 127 & 5.9 & mixture & -121 & -16.14 & 28.8 & .96 \\
\hline 45 & .20 & 8.90 & $<.002$ & $<.06$ & .02 & .007 & 260 & 2,021 & 118.1 & reduced & -128 & -17.22 & 36.2 & .96 \\
\hline 46 & .18 & 12.5 & $<.002$ & .63 & $<.02$ & .007 & 134 & 115 & 21.2 & mixture & -125 & -16.83 & 30.4 & .96 \\
\hline
\end{tabular}


Table 2. Selected well-construction, major-ion, nutrient, stable isotopes of hydrogen and oxygen, and tritium data from groundwater and surface water, Eagle River watershed, 2006-2007, Eagle County, Colorado.-Continued

\begin{tabular}{|c|c|c|c|c|c|c|c|c|c|c|c|c|c|c|}
\hline $\begin{array}{c}\text { Local } \\
\text { identification } \\
\text { number }\end{array}$ & $\begin{array}{c}\text { Fluoride, } \\
\text { dissolved } \\
\text { (mg/L) }\end{array}$ & $\begin{array}{c}\text { Silica, } \\
\text { dissolved } \\
\text { (mg/L) }\end{array}$ & $\begin{array}{l}\text { Nitrite, } \\
\text { dissolved } \\
\text { (mg/L) }\end{array}$ & $\begin{array}{c}\text { Nitrite plus } \\
\text { nitrate, dissolved, } \\
\text { as nitrogen } \\
\text { (mg/L) }\end{array}$ & $\begin{array}{l}\text { Ammonia, } \\
\text { dissolved } \\
\text { (mg/L) }\end{array}$ & $\begin{array}{l}\text { Orthophosphate, } \\
\text { dissolved } \\
\text { (mg/L) }\end{array}$ & $\begin{array}{c}\text { Boron, } \\
\text { dissolved } \\
(\mu \mathrm{g} / \mathrm{L})\end{array}$ & $\begin{array}{c}\text { Iron, } \\
\text { dissolved } \\
(\mu \mathrm{g} / \mathrm{L})\end{array}$ & $\begin{array}{l}\text { Manganese, } \\
\text { dissolved } \\
(\mu \mathrm{g} / \mathrm{L})\end{array}$ & $\begin{array}{l}\text { Oxidation } \\
\text { state }\end{array}$ & $\begin{array}{l}\text { Hydrogen } \\
2 / 1 \text { ratio } \\
\text { (per mil) }\end{array}$ & $\begin{array}{l}\text { Oxygen } \\
\text { 18/16 ratio } \\
\text { (per mil) }\end{array}$ & $\begin{array}{l}\text { Tritium } \\
\text { (pCi/L) }\end{array}$ & $\begin{array}{c}\text { Tritium, } 2 \text { sigma } \\
\text { precision } \\
\text { estimate } \\
\text { (pCi/L) }\end{array}$ \\
\hline 47 & .49 & 14.8 & $<.002$ & 5.42 & e .02 & .011 & 328 & 34 & $<3.0$ & oxidized & -140 & -17.71 & 4.00 & .38 \\
\hline 47 & .48 & 14.6 & $<.002$ & 5.39 & e .02 & .008 & 308 & 28 & e 1.0 & oxidized & -140 & -17.8 & 4.9 & .42 \\
\hline 48 & .23 & 14.8 & $<.002$ & .95 & $<.02$ & .006 & 35 & 16 & .4 & oxidized & -124 & -16.89 & 29.4 & .96 \\
\hline 49 & .19 & 13.9 & $<.002$ & 2.39 & $<.02$ & e .003 & 106 & 20 & e .4 & oxidized & -127 & -16.92 & 32.3 & .96 \\
\hline 50 & .44 & 25.4 & $<.002$ & 1.49 & e .01 & .019 & 62 & $<18$ & $<1.8$ & oxidized & -128 & -17.48 & 28.0 & .86 \\
\hline 51 & .18 & 12.8 & $<.002$ & $<.06$ & $<.02$ & $<.006$ & 45 & 108 & 90.7 & mixture & -125 & -16.98 & 27.8 & .96 \\
\hline 52 & .28 & 17.3 & $<.002$ & .59 & $<.02$ & .010 & 88 & 9 & e .5 & oxidized & -130 & -17.31 & 2.02 & .35 \\
\hline 53 & .21 & 9.35 & $<.002$ & $<.06$ & .03 & $<.006$ & 171 & 545 & 88.9 & reduced & -131 & -17.65 & 1.50 & .61 \\
\hline 54 & .26 & 14.0 & $<.002$ & 1.77 & $<.02$ & .006 & 98 & e 4 & $\mathrm{e} .2$ & oxidized & -131 & -17.38 & 10.5 & .70 \\
\hline 55 & .25 & 17.6 & $<.002$ & 1.27 & $<.02$ & .007 & 64 & e 11 & .6 & oxidized & -125 & -16.89 & 24.3 & .83 \\
\hline 56 & .17 & 17.9 & $<.002$ & .88 & $<.02$ & .006 & 48 & 14 & 1.0 & oxidized & -124 & -16.89 & 28.2 & .93 \\
\hline 57 & .22 & 17.1 & $<.002$ & .78 & $<.02$ & .009 & 54 & 113 & 3.9 & mixture & -124 & -16.90 & 33.9 & .96 \\
\hline 58 & .37 & 14.0 & $<.002$ & 2.86 & $<.02$ & e .005 & 155 & e 14 & $<.6$ & oxidized & -128 & -17.19 & 36.8 & .96 \\
\hline 59 & .52 & 12.8 & $<.002$ & .74 & $<.02$ & .007 & 95 & e 3 & 53.1 & mixture & -124 & -17.12 & 37.1 & 1.31 \\
\hline 60 & .12 & 13.6 & $<.002$ & .26 & $<.02$ & .006 & 21 & 65 & 1.7 & oxidized & -125 & -17.09 & 29.8 & .96 \\
\hline 61 & e .10 & 15.3 & .002 & 1.25 & $<.02$ & e .005 & 39 & 535 & 18.8 & mixture & -123 & -16.81 & 32.6 & 1.28 \\
\hline 62 & .22 & 14.4 & $<.002$ & $<.06$ & .60 & .016 & 176 & 3,811 & $1,500.0$ & reduced & -134 & -17.93 & 42.9 & 1.31 \\
\hline 63 & .27 & 14.0 & $<.002$ & 1.64 & .03 & .008 & 257 & e 17 & 116.3 & mixture & -130 & -17.39 & 26.2 & .96 \\
\hline 64 & .23 & 16.1 & .007 & 1.10 & $<.02$ & .008 & 103 & 129 & 166.0 & mixture & -124 & -16.73 & 35.2 & 1.28 \\
\hline 65 & .27 & 23.8 & .003 & $<.06$ & 1.97 & .110 & 227 & 8,364 & 993.0 & reduced & -131 & -17.21 & 20.0 & .70 \\
\hline 66 & e .09 & 5.36 & -- & -- & -- & -- & 6 & 13 & 12.1 & na & -126 & -17.27 & 27.2 & .96 \\
\hline 66 & e .08 & 5.34 & -- & -- & -- & -- & 9 & 11 & 15.3 & na & -130 & -17.78 & 19.8 & .96 \\
\hline 66 & e .06 & 4.79 & -- & -- & -- & -- & 4 & 15 & 5.2 & na & -126 & -17.38 & 31.0 & .96 \\
\hline 66 & .10 & 4.46 & -- & -- & -- & -- & 4 & 18 & 8.1 & na & -119 & -16.24 & 28.5 & 2.59 \\
\hline 69 & .10 & 4.46 & .007 & .54 & e .01 & .081 & -- & -- & -- & na & -125 & -17.06 & 30.0 & .93 \\
\hline 70 & .14 & 6.50 & .005 & .40 & e. 02 & .020 & -- & -- & 59.8 & na & -129 & -17.22 & 29.8 & 1.28 \\
\hline 70 & e .07 & 6.32 & .002 & .35 & $<.02$ & .013 & -- & -- & -- & na & -130 & -17.48 & 30.4 & 1.28 \\
\hline 70 & e .06 & 5.49 & .001 & .08 & $<.02$ & e .003 & -- & -- & 13.0 & na & -128 & -17.38 & 30.7 & 1.28 \\
\hline 70 & e .09 & 4.74 & .002 & .11 & $<.02$ & .009 & -- & -- & 13.3 & na & -120 & -16.31 & 28.2 & 2.59 \\
\hline 71 & .11 & 4.43 & .008 & .74 & .02 & .087 & -- & -- & -- & na & -- & -- & -- & -- \\
\hline 71 & -- & -- & -- & -- & -- & -- & -- & -- & -- & na & -122 & -16.42 & 28.0 & .86 \\
\hline 71 & .17 & 6.55 & .014 & 1.34 & .03 & .165 & -- & -- & 22.3 & na & -126 & -17.02 & 29.1 & .96 \\
\hline 71 & e .09 & 5.83 & .006 & .64 & e .01 & .063 & -- & -- & -- & na & -128 & -17.27 & 27.8 & .96 \\
\hline 71 & $<.10$ & 5.27 & .001 & .14 & $<.02$ & .008 & -- & -- & 8.8 & na & -126 & -17.23 & 31.7 & 1.28 \\
\hline 71 & .12 & 4.02 & .005 & .61 & $<.02$ & .073 & -- & -- & 7.9 & na & -121 & -16.28 & 29.4 & 2.59 \\
\hline 72 & e .06 & 9.25 & -- & -- & -- & -- & 11 & e 5 & 3.2 & na & -128 & -17.39 & 28.8 & .96 \\
\hline 72 & e .05 & 9.06 & -- & -- & -- & -- & 10 & 10 & 5.5 & na & -130 & -17.48 & 30.7 & .96 \\
\hline 72 & $<.10$ & 6.90 & -- & -- & -- & -- & 7 & 20 & 3.5 & na & -124 & -16.90 & 28.8 & .96 \\
\hline 72 & e .07 & 8.73 & -- & -- & -- & -- & 9 & e 4 & 5.7 & na & -125 & -16.91 & 29.4 & 2.59 \\
\hline 73 & $<.10$ & 9.40 & -- & -- & -- & -- & 11 & e 4 & 3.8 & na & -126 & -17.20 & 33.9 & .96 \\
\hline 73 & e .05 & 9.15 & -- & -- & -- & -- & 12 & e 6 & 5.7 & na & -127 & -17.30 & 33.0 & .96 \\
\hline 73 & $<.10$ & 7.35 & -- & -- & -- & -- & 6 & e 6 & 5.3 & na & -123 & -16.91 & 31.4 & .96 \\
\hline 73 & $<.10$ & 9.04 & -- & -- & -- & -- & 10 & e 5 & 2.1 & na & -125 & -17.03 & 32.0 & 2.59 \\
\hline 74 & .16 & 6.65 & .007 & 1.04 & e .02 & .103 & -- & -- & 11.5 & na & -125 & -16.92 & 27.8 & .96 \\
\hline 74 & .15 & 6.36 & .011 & .93 & .03 & .098 & -- & -- & -- & na & -128 & -17.17 & 28.8 & .96 \\
\hline 74 & $<.10$ & 5.45 & .002 & .14 & $<.02$ & .007 & -- & -- & 7.7 & na & -125 & -17.14 & 33.3 & .96 \\
\hline 74 & .15 & 4.71 & .004 & .29 & $<.02$ & .038 & -- & -- & 19.6 & na & -121 & -16.22 & 28.8 & 2.59 \\
\hline
\end{tabular}


Table 3. Selected ground- and surface-water-quality data used to interpret groundwater recharge dates using chlorofluorocarbons, Eagle River watershed, Eagle County, Colorado.

[gw, groundwater site; sw, surface-water site; m, month; d, day; y, year; cc/kg, cubic centimeters per kilogram; ${ }^{\circ} \mathrm{C}$, degrees Celsius; NGVD 29, National Geodetic Vertical Datum of 1929; CFC, chlorofluorocarbon; mean concentration, mean CFC concentration normally calculated from three replicate samples collected at each site; pg/kg, picograms per kilogram; ppt, parts per trillion; pf, piston flow; na, not applicable]

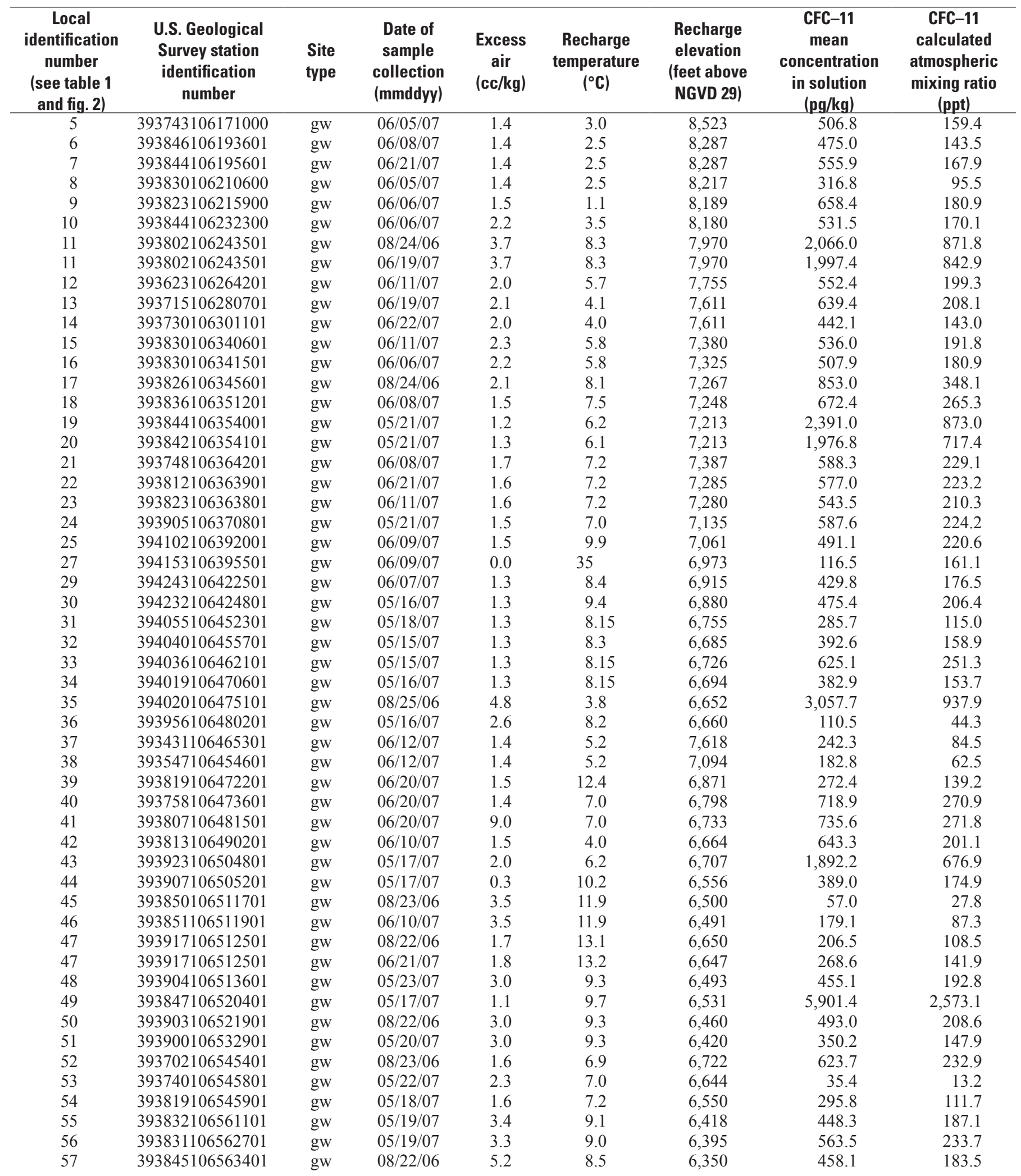


Table 3. Selected ground- and surface-water-quality data used to interpret groundwater recharge dates using chlorofluorocarbons, Eagle River watershed, Eagle County, Colorado.-Continued

[gw, groundwater site; sw, surface-water site; m, month; d, day; y, year; cc/kg, cubic centimeters per kilogram; ${ }^{\circ} \mathrm{C}$, degrees Celsius; NGVD 29, National Geodetic Vertical Datum of 1929; CFC, chlorofluorocarbon; mean concentration, mean CFC concentration normally calculated from three replicate samples collected at each site; pg/kg, picograms per kilogram; ppt, parts per trillion; pf, piston flow; na, not applicable]

\begin{tabular}{|c|c|c|c|c|c|c|c|}
\hline $\begin{array}{c}\text { Local } \\
\text { identification } \\
\text { number } \\
\text { (see table } 1 \\
\text { and fig. 2) }\end{array}$ & $\begin{array}{c}\text { CFC-12 } \\
\text { mean } \\
\text { concentration } \\
\text { in solution } \\
(\mathrm{pg} / \mathrm{kg}) \\
\end{array}$ & $\begin{array}{c}\text { CFC-12 } \\
\text { calculated } \\
\text { atmospheric } \\
\text { mixing ratio } \\
\text { (ppt) }\end{array}$ & $\begin{array}{c}\text { CFC-113 } \\
\text { mean } \\
\text { concentration } \\
\text { in solution } \\
(\mathrm{pg} / \mathrm{kg}) \\
\end{array}$ & $\begin{array}{c}\text { CFC-113 } \\
\text { calculated } \\
\text { atmospheric } \\
\text { mixing ratio } \\
\text { (ppt) }\end{array}$ & $\begin{array}{c}\text { Mean } \\
\text { groundwater } \\
\text { recharge } \\
\text { date of young } \\
\text { fraction } \\
\end{array}$ & $\begin{array}{c}\text { Percentage } \\
\text { of young } \\
\text { water }\end{array}$ & $\begin{array}{l}\text { Mean } \\
\text { groundwater } \\
\text { recharge date } \\
\text { based on: }\end{array}$ \\
\hline 5 & 714.0 & $1,019.1$ & 98.0 & 70.0 & 1989 & 100 & CFC-113 pf \\
\hline 6 & 864.0 & $1,188.5$ & 40.9 & 28.0 & 1984 & 73 & CFC-113/CFC-11 ratio \\
\hline 7 & 955.9 & $1,314.9$ & 51.2 & 35.1 & 1985 & 79 & CFC-113/CFC-11 ratio \\
\hline 10 & $1,146.2$ & $1,651.0$ & 73.5 & 53.4 & 1996 & 63 & CFC-113/CFC-11 ratio \\
\hline 11 & $2,449.3$ & $4,447.3$ & 57.0 & 55.4 & 1987 & 100 & CFC-113 pf \\
\hline 11 & $1,859.7$ & $3,376.7$ & 68.1 & 66.1 & 1988 & 100 & CFC-113 pf \\
\hline 12 & 374.1 & 598.9 & 164.6 & 136.4 & 1983 & 100 & CFC-11 pf \\
\hline 13 & 425.0 & 620.2 & 161.6 & 119.8 & 1984 & 100 & $\mathrm{CFC}-11 \mathrm{pf}$ \\
\hline 14 & 379.4 & 551.1 & 68.3 & 50.3 & 1986 & 100 & CFC -113 pf \\
\hline 19 & $1,982.4$ & $3,217.2$ & 97.2 & 82.0 & 1992 & 100 & CFC-113 pf \\
\hline 20 & $174,625.7$ & $281,769.9$ & 75.0 & 62.9 & 1988 & 100 & CFC -113 pf \\
\hline 21 & $1,255.2$ & $2,150.8$ & 67.1 & 60.5 & 1989 & 91 & CFC-113/CFC-11 ratio \\
\hline 22 & 571.8 & 974.5 & 75.8 & 68.0 & 1994 & 83 & CFC-113/CFC-11 ratio \\
\hline 23 & 531.6 & 905.7 & 73.5 & 65.9 & 1991 & 76 & CFC-113/CFC-11 ratio \\
\hline 24 & 663.0 & $1,116.0$ & 75.0 & 66.3 & 1989 & 84 & CFC-113/CFC-11 ratio \\
\hline 25 & 318.9 & 620.1 & 62.3 & 65.7 & 1992 & 83 & CFC-113/CFC-11 ratio \\
\hline 27 & 260.5 & $1,404.9$ & 48.3 & 179.8 & $<1940$ & 0 & CFC-11 pf \\
\hline 29 & 297.9 & 535.9 & 27.6 & 26.5 & 1981 & 100 & CFC-113 pf \\
\hline 30 & 255.0 & 481.7 & 39.8 & 40.6 & 1985 & 100 & CFC-113 pf \\
\hline 31 & 239.8 & 423.2 & 32.5 & 30.6 & 1989 & 46 & CFC-113/CFC-11 ratio \\
\hline 32 & 544.0 & 964.9 & 55.4 & 52.4 & 1996 & 61 & CFC-113/CFC-11 ratio \\
\hline 41 & 444.5 & 690.9 & 85.7 & 70.8 & 1989 & 100 & CFC -113 pf \\
\hline 42 & 400.2 & 563.7 & 76.4 & 54.5 & 1989 & 80 & CFC-113/CFC-11 ratio \\
\hline 43 & 445.2 & 704.3 & 63.1 & 51.9 & 1986 & 100 & CFC-113 pf \\
\hline 44 & 276.6 & 542.7 & 51.6 & 55.1 & 1991 & 73 & CFC-113/CFC-11 ratio \\
\hline 45 & 23.2 & 47.5 & 34.4 & 39.3 & 1965 & 100 & $\mathrm{CFC}-11 \mathrm{pf}$ \\
\hline 46 & 309.3 & 634.4 & 23.1 & 26.4 & 1992 & 32 & CFC-113/CFC-11 ratio \\
\hline 47 & 171.7 & 382.2 & 27.7 & 34.7 & 1996 & 41 & CFC-113/CFC-11 ratio \\
\hline 47 & 223.6 & 499.7 & 36.4 & 45.8 & 1995 & 54 & CFC-113/CFC-11 ratio \\
\hline 48 & 309.5 & 563.6 & 60.5 & 59.6 & 1995 & 72 & CFC-113/CFC-11 ratio \\
\hline 49 & 339.1 & 642.9 & 51.0 & 52.3 & 1986 & 100 & CFC -113 pf \\
\hline 50 & 323.9 & 589.2 & 68.0 & 66.8 & 1996 & 79 & CFC-113/CFC-11 ratio \\
\hline 51 & 318.6 & 578.7 & 52.8 & 51.9 & 1992 & 47 & CFC-113/CFC-11 ratio \\
\hline 52 & 339.6 & 559.6 & 76.7 & 66.3 & 1990 & 90 & CFC-113/CFC-11 ratio \\
\hline 53 & 255.4 & 419.2 & 7.1 & 6.1 & 1990 & 2 & CFC-113/CFC-11 ratio \\
\hline 54 & 235.9 & 392.3 & 31.0 & 27.1 & 1988 & 47 & CFC-113/CFC-11 ratio \\
\hline 55 & 333.5 & 597.6 & 63.1 & 61.0 & 1998 & 72 & CFC-113/CFC-11 ratio \\
\hline 56 & 382.0 & 681.2 & 64.6 & 62.0 & 1989 & 93 & CFC-113/CFC-11 ratio \\
\hline 57 & 382.2 & 652.2 & 60.8 & 55.7 & 1993 & 68 & CFC-113/CFC-11 ratio \\
\hline
\end{tabular}


Table 3. Selected ground- and surface-water-quality data used to interpret groundwater recharge dates using chlorofluorocarbons, Eagle River watershed, Eagle County, Colorado.-Continued

[gw, groundwater site; sw, surface-water site; m, month; d, day; y, year; cc/kg, cubic centimeters per kilogram; ${ }^{\circ} \mathrm{C}$, degrees Celsius; NGVD 29 , National Geodetic Vertical Datum of 1929; CFC, chlorofluorocarbon; mean concentration, mean CFC concentration normally calculated from three replicate samples collected at each site; pg/kg, picograms per kilogram; ppt, parts per trillion; pf, piston flow; na, not applicable]

\begin{tabular}{|c|c|c|c|c|c|c|c|c|}
\hline $\begin{array}{c}\text { Local } \\
\text { identification } \\
\text { number } \\
\text { (see table } 1 \\
\text { and fig. 2) }\end{array}$ & $\begin{array}{c}\text { U.S. Geological } \\
\text { Survey station } \\
\text { identification } \\
\text { number }\end{array}$ & $\begin{array}{l}\text { Site } \\
\text { type }\end{array}$ & $\begin{array}{l}\text { Date of } \\
\text { sample } \\
\text { collection } \\
\text { (mmddyy) }\end{array}$ & $\begin{array}{c}\text { Excess } \\
\text { air } \\
(\mathrm{cc} / \mathrm{kg})\end{array}$ & $\begin{array}{c}\text { Recharge } \\
\text { temperature } \\
\left({ }^{\circ} \mathrm{C}\right)\end{array}$ & $\begin{array}{c}\text { Recharge } \\
\text { elevation } \\
\text { (feet above } \\
\text { NGVD 29) }\end{array}$ & $\begin{array}{c}\text { CFC-11 } \\
\text { mean } \\
\text { concentration } \\
\text { in solution } \\
(\mathrm{pg} / \mathrm{kg})\end{array}$ & $\begin{array}{c}\text { CFC-11 } \\
\text { calculated } \\
\text { atmospheric } \\
\text { mixing ratio } \\
\text { (ppt) }\end{array}$ \\
\hline 58 & 393554106564901 & gw & $05 / 20 / 07$ & 1.5 & 11.2 & 6,760 & 252.4 & 120.4 \\
\hline 59 & 393632106581101 & gw & $08 / 23 / 06$ & 3.5 & 4.9 & 6,620 & 673.4 & 221.3 \\
\hline 60 & 393658106574101 & gw & $05 / 20 / 07$ & 1.2 & 1.9 & 6,528 & 447.6 & 121.9 \\
\hline 63 & 393900107030701 & gw & $05 / 19 / 07$ & 2.8 & 11.3 & 6,152 & 42.1 & 19.7 \\
\hline 64 & 393902107033901 & gw & $05 / 22 / 07$ & 2.7 & 11.1 & 6,155 & 627.1 & 290.0 \\
\hline 65 & 393825107050301 & gw & $05 / 22 / 07$ & 0.0 & 11.7 & 8,545 & 8.4 & 4.4 \\
\hline 66 & 393737106165900 & sw & $05 / 23 / 07$ & 0.0 & 3.5 & 8,545 & 669.7 & 218.2 \\
\hline 66 & 393737106165900 & sw & $08 / 14 / 07$ & 0.0 & 13.5 & 8,545 & 387.2 & 224.4 \\
\hline 67 & 393834106230401 & sw & $08 / 14 / 07$ & 0.0 & 14.7 & 8,120 & 545.4 & 331.0 \\
\hline 71 & 394220106431500 & sw & $08 / 25 / 06$ & 0.0 & 18.0 & 6,780 & 338.7 & 230.3 \\
\hline 71 & 394220106431500 & sw & $08 / 14 / 07$ & 0.0 & 19.9 & 6,820 & 299.3 & 222.9 \\
\hline 72 & 09068000 & sw & $05 / 23 / 07$ & 0.0 & 3.9 & 7,413 & 609.2 & 195.2 \\
\hline 72 & 09068000 & SW & $08 / 14 / 07$ & 0.0 & 14.8 & 7,413 & 370.7 & 220.2 \\
\hline 73 & 09069500 & sw & $05 / 23 / 07$ & 0.0 & 8 & 7,600 & 613.1 & 253.2 \\
\hline 73 & 09069500 & SW & $08 / 14 / 07$ & 0.0 & 13.8 & 7,600 & 395.6 & 224.8 \\
\hline 74 & 09069000 & sw & $05 / 23 / 07$ & 0.0 & 5.9 & 6,276 & 621.8 & 215.9 \\
\hline 74 & 09069000 & sw & $08 / 14 / 07$ & 0.0 & 20.1 & 6,276 & 281.1 & 207.0 \\
\hline 75 & 09070400 & sw & $08 / 23 / 06$ & 0.0 & 19.4 & 6,150 & 487.4 & 345.7 \\
\hline 75 & 09070400 & sw & $05 / 23 / 07$ & 0.0 & 5.9 & 6,150 & 613.6 & 212.1 \\
\hline
\end{tabular}

CFCs provide excellent tracers and dating tools of young water (50-year time scale). Groundwater age dating with CFCs is based on Henry's law solubility, which is the concentration of the gas dissolved in water in equilibrium with air proportional to the partial pressure of the gas in air (Plummer and Busenberg, 1999). Under favorable conditions, sufficient concentrations of CFC-12, CFC-11, and CFC-113 can dissolve into water and allow dating of groundwater recharged since approximately 1941, 1947, and 1955, respectively. Groundwater age dating with $\mathrm{CFC}-12$, $\mathrm{CFC}-11$, and $\mathrm{CFC}-113$ is possible because (1) the atmospheric mixing ratios (concentrations of the compounds in air) of these compounds are known and(or) have been reconstructed over the past 50 years, (2) the Henry's law solubilities in water are known, and (3) concentrations in air and young water are relatively high and can be measured (Plummer and Busenberg, 1999). Atmospheric concentrations of CFC-12, CFC-11, and CFC-113 in air peaked in about 2001, 1994, and 1996, respectively (fig. 3). Because of nearly level atmospheric input functions of CFC mixing ratios in the 1990s and early 2000s, it is not possible to resolve modern CFC ages as precisely as can be done with waters recharged earlier, when atmospheric concentrations were increasing rapidly (Plummer and others, 2006).

\section{Dissolved Gases}

Groundwater age dating with CFCs is based on gas solubility, which is affected by the recharge temperature and excess air in the water sample and the altitude at the location of recharge (Plummer and Busenberg, 1999). The recharge temperature is the temperature of the recharge water at the water table during recharge; overestimation of recharge temperature results in apparent ages that are too young, and underestimation gives apparent ages that are too old. Dissolved gases of argon (Ar), carbon dioxide $\left(\mathrm{CO}_{2}\right)$, methane $\left(\mathrm{CH}_{4}\right)$, nitrogen $\left(\mathrm{N}_{2}\right)$, and oxygen $\left(\mathrm{O}_{2}\right)$ in groundwater were used to estimate recharge temperature and excess air of the water samples. The concentration, as well as the ratios of concentrations of dissolved gases, can be used to calculate the recharge temperature of a groundwater sample using Henry's law. Excess air is air dissolved in groundwater in relative proportions equal to that of the troposphere and in excess of that determined by solubility equilibrium. Excess air is trapped and dissolved under increased hydrostatic pressure in groundwater at the capillary fringe or in fractures as the water table rises. High concentrations of excess air are common in fractured rock 
Table 3. Selected ground- and surface-water-quality data used to interpret groundwater recharge dates using chlorofluorocarbons, Eagle River watershed, Eagle County, Colorado.-Continued

[gw, groundwater site; sw, surface-water site; m, month; d, day; y, year; cc/kg, cubic centimeters per kilogram; ${ }^{\circ} \mathrm{C}$, degrees Celsius; NGVD 29, National Geodetic Vertical Datum of 1929; CFC, chlorofluorocarbon; mean concentration, mean CFC concentration normally calculated from three replicate samples collected at each site; pg/kg, picograms per kilogram; ppt, parts per trillion; pf, piston flow; na, not applicable]

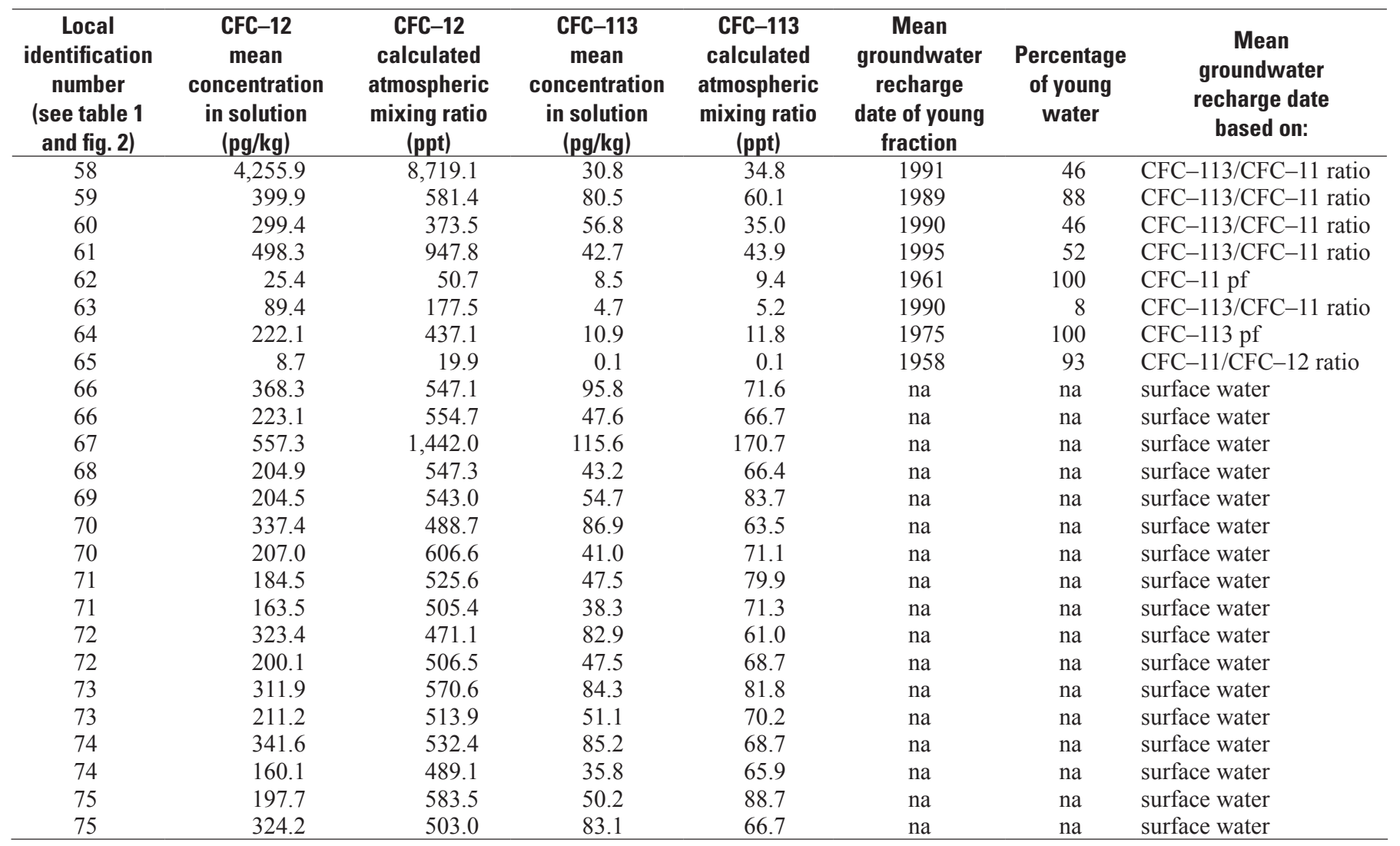

aquifers and in aquifers in semiarid areas. Introduction of excess air adds CFCs to groundwater and, if not accounted for in age interpretation, causes a young-age bias (Plummer and Busenberg, 1999). Excess air is determined by comparing the ratio of $\mathrm{N}_{2}$ to $\mathrm{Ar}$ in air to the equilibrium solubility-ratio of $\mathrm{N}_{2}$ to $\mathrm{Ar}$ in water. In most cases, the altitude at location of recharge was estimated to be the altitude of the land surface at the wellhead.

\section{Low-Level Volatile Organic Compounds}

Recently, the USGS developed an analytical procedure (Plummer and others, 2008) to determine concentrations of 25 halogenated VOCs at minimum detection levels (MDLs) that typically are 2 to more than 4 orders of magnitude below the USGS purge and trap capillary column gas chromatograph-mass spectrometer (GC-MS) procedure (Rose and Schroeder, 1995; Connor and others, 1998). The GC-MS procedure corresponds closely to USEPA method 524 for drinking-water samples (Moran and others, 2006). The new analytical procedure for halogenated VOCs uses purge and trap gas chromatography with electron-capture detector (GC-ECD), similar to that of Busenberg and Plummer (1992). Details of the low-level VOC sampling and analyses from the USGS Chlorofluorocarbon Laboratory are given at http://water.usgs.gov/lab/chlorofluorocarbons/lab/ analytical procedures/ and in Plummer and others (2008). "Low-level" detections of CFCs and other halogenated VOCs can be quite useful in determining whether fractions of post1940s water are present in groundwater samples. The extremely low MDLs allow post-1940s water to be identified more frequently than it would be by using GC-MS VOC analysis.

Concentrations of 15 halogenated VOCs were determined in the Eagle County groundwaters by the GC-ECD analytical procedure (table 5). Although the VOC concentrations typically are very low and present no known health risk, their detections indicate post-1940s recharge, or mixtures of recharge that contain a fraction of post-1940s water. Concentrations of the 15 halogenated VOCs in groundwater expected from natural and anthropogenic atmospheric sources were estimated and used to recognize water samples that have VOC concentrations exceeding the natural and anthropogenic atmospheric threshold and, thus, identify those samples that are being affected from nonatmospheric sources. 
Table 4. Mean concentrations of low-level volatile organic compounds detected in groundwater and surface water of the Eagle River watershed, 2006-2007, Eagle County, Colorado.

[m, month; d, day; y, year; gw, groundwater site; sw, surface-water site; --, not detected; pg/L, picograms per liter]

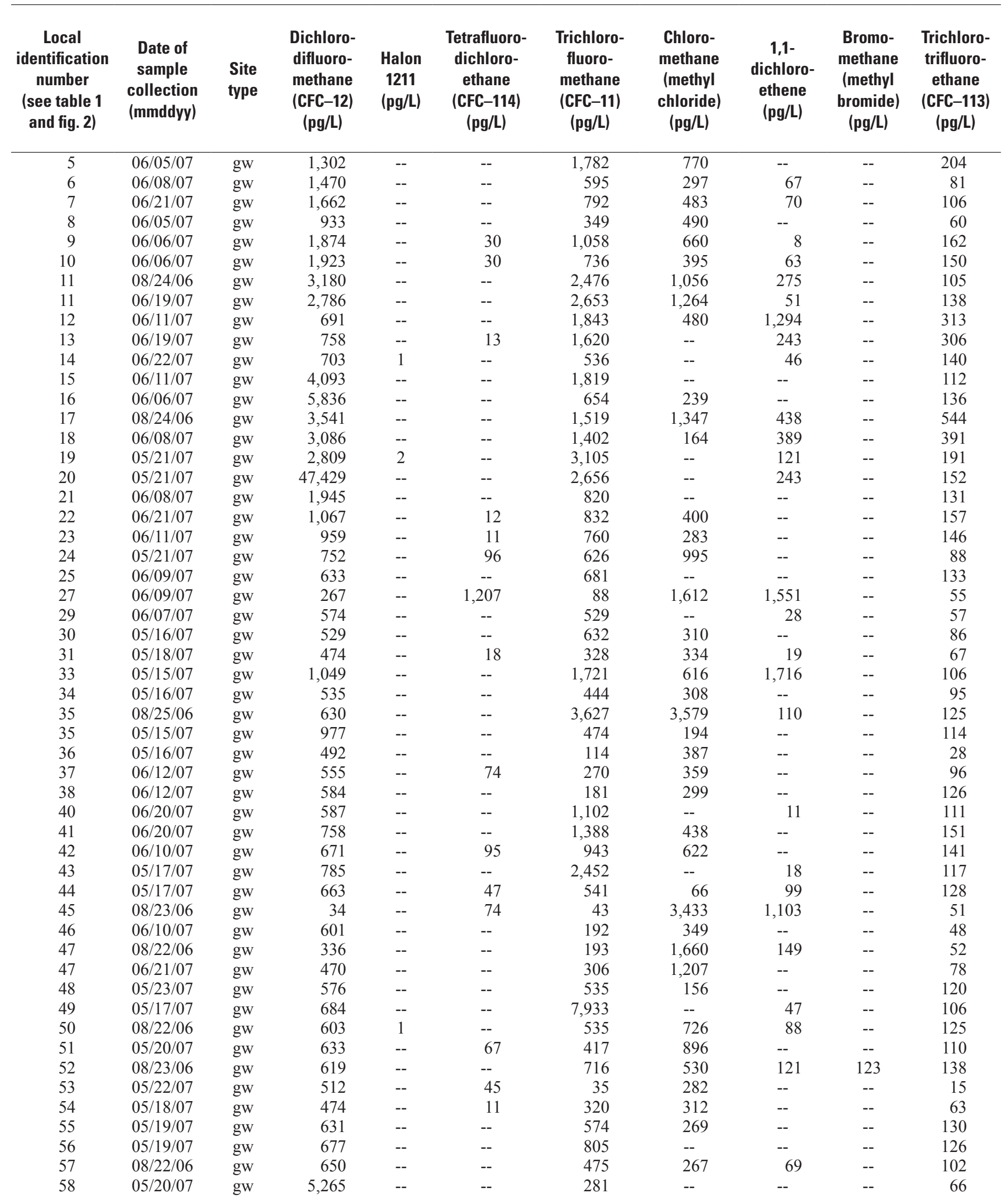


Table 4. Mean concentrations of low-level volatile organic compounds detected in groundwater and surface water of the Eagle River watershed, 2006-2007, Eagle County, Colorado.-Continued

[m, month; d, day; y, year; gw, groundwater site; sw, surface-water site; --, not detected; pg/L, picograms per liter]

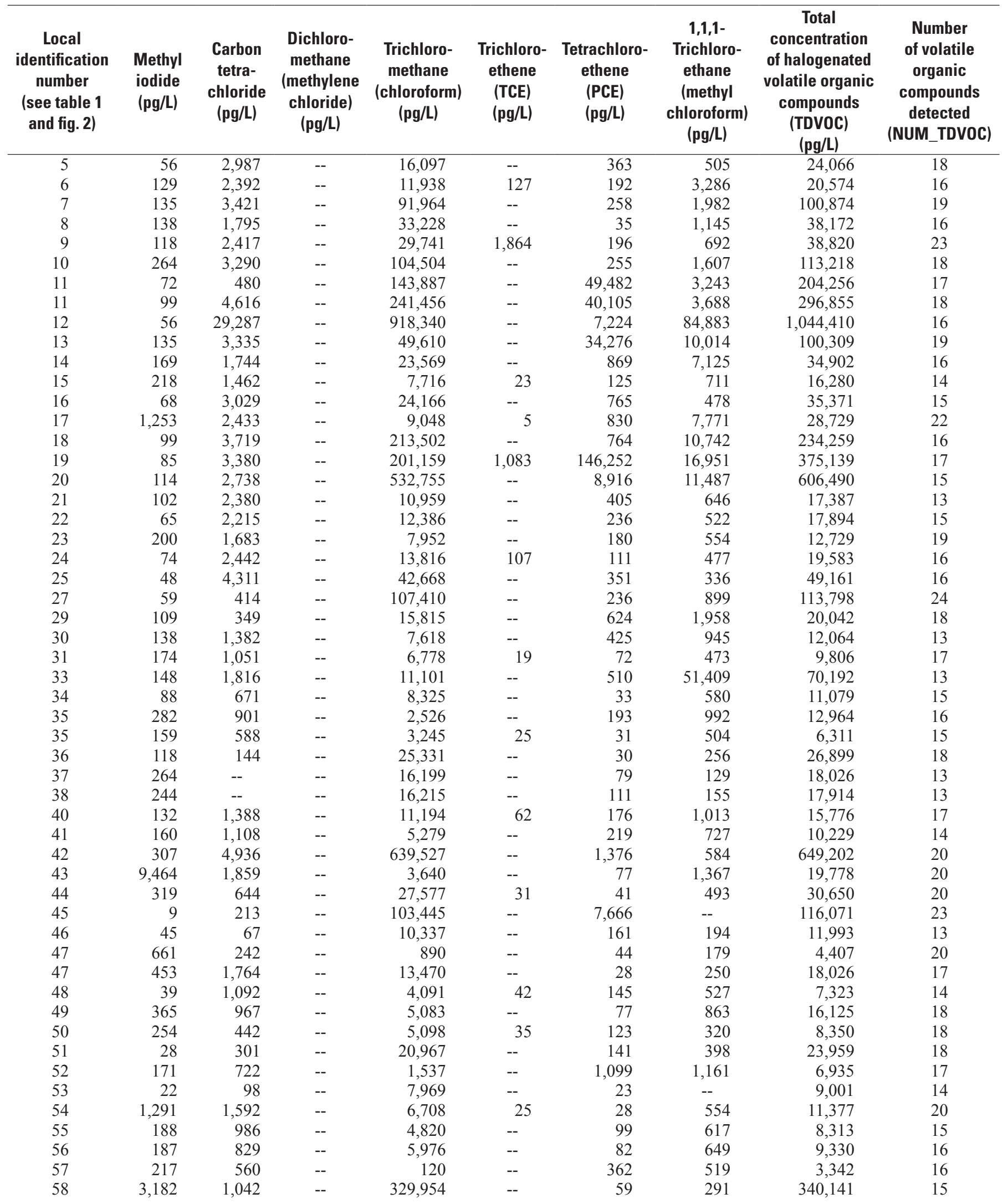


Table 4. Mean concentrations of low-level volatile organic compounds detected in groundwater and surface water of the Eagle River watershed, 2006-2007, Eagle County, Colorado.-Continued

[m, month; d, day; y, year; gw, groundwater site; sw, surface-water site; --, not detected; pg/L, picograms per liter]

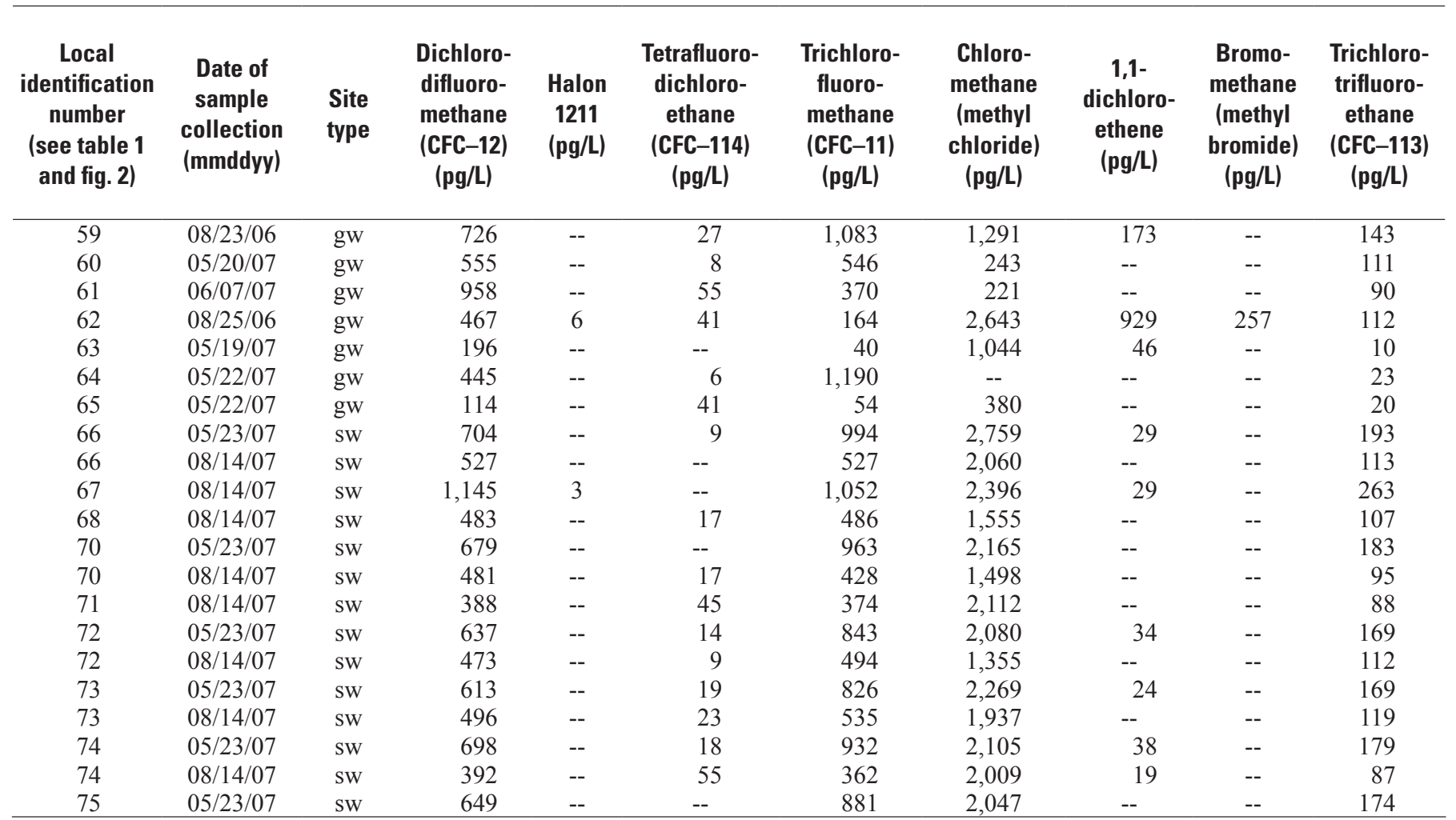

\section{Geographic Information System Data}

Groundwater quality data were correlated with hydrogeologic data to develop the groundwater probability models and maps. Hydrogeologic data consisted of GIS data on depth to groundwater, geology, hydrography, precipitation, and soils.

The boundary of the ERWVFA was developed by combining information from two data sources. The first data source was a 1:250,000-scale geologic map of the Leadville quadrangle developed by Day and others (1999). The location of Quaternary sediments was used as a first approximation of the ERWVFA. The boundary of the ERWVFA was further refined by overlaying the geologic map with Digital Raster Graphic (DRG) scanned images of 1:24,000 topographic maps (U.S. Geological Survey, 2001). Where appropriate, the boundary of the ERWVFA was remapped to correspond with the abrupt change in topography at the edge of the valley floor throughout the Eagle River watershed. The boundary of the ERWVFA more closely resembles a hydrogeomorphic region presented by Rupert (2003, p. 8) because it is based upon general geographic locations of geologic materials and not on an actual aquifer location determined through rigorous hydrogeologic investigation.
A depth-to-groundwater GIS data layer was generated by subtracting a water-table map of groundwater in the ERWVFA from $10 \mathrm{~m}(32.8 \mathrm{ft})$ National Elevation Dataset (NED) data (U.S. Geological Survey, 1999a). The watertable map was generated by hand-contouring groundwater levels measured in 117 wells during May and June 2003 (http://waterdata.usgs.gov/co/nwis/gw). Because of inaccuracies in the NED data, a few riparian areas had negative depths to groundwater of a few feet because of the high water table. In this case, depth to groundwater was set at $0.3 \mathrm{~m}(1 \mathrm{ft})$ below land surface.

Geology data were developed by Day and others (1999), who produced a 1:250,000-scale geologic map of the Leadville quadrangle. Geologic units of interest include the Quaternary sediments, which were used to map the boundary of the ERWVFA, and the Eagle Valley Evaporite, which delineates the location of the gypsum beds in the area. Distance of wells from the gypsum beds was used as one of the independent variables in the logistic regression modeling.

The hydrography data in the Eagle River watershed were extracted from the National Hydrography Dataset (NHD) (U.S. Geological Survey, 1999b). The NHD data were edited to include only the major canals, rivers, and streams. Distance of wells from the major canals, rivers, and streams was used as one of the independent variables in the logistic regression modeling. 
Table 4. Mean concentrations of low-level volatile organic compounds detected in groundwater and surface water of the Eagle River watershed, 2006-2007, Eagle County, Colorado.-Continued

[m, month; d, day; y, year; gw, groundwater site; sw, surface-water site; --, not detected; pg/L, picograms per liter]

\begin{tabular}{|c|c|c|c|c|c|c|c|c|c|}
\hline $\begin{array}{c}\text { Local } \\
\text { identification } \\
\text { number } \\
\text { (see table } 1 \\
\text { and fig. 2) }\end{array}$ & $\begin{array}{l}\text { Methyl } \\
\text { iodide } \\
\text { (pg/L) }\end{array}$ & $\begin{array}{c}\text { Carbon } \\
\text { tetra- } \\
\text { chloride } \\
(\mathrm{pg} / \mathrm{L})\end{array}$ & $\begin{array}{l}\text { Dichloro- } \\
\text { methane } \\
\text { (methylene } \\
\text { chloride) } \\
\text { (pg/L) }\end{array}$ & $\begin{array}{c}\text { Trichloro- } \\
\text { methane } \\
\text { (chloroform) } \\
\text { (pg/L) }\end{array}$ & $\begin{array}{l}\text { Trichloro- } \\
\text { ethene } \\
\text { (TCE) } \\
\text { (pg/L) }\end{array}$ & $\begin{array}{l}\text { Tetrachloro- } \\
\text { ethene } \\
\text { (PCE) } \\
\text { (pg/L) }\end{array}$ & $\begin{array}{c}\text { 1,1,1- } \\
\text { Trichloro- } \\
\text { ethane } \\
\text { (methyl } \\
\text { chloroform) } \\
\text { (pg/L) }\end{array}$ & $\begin{array}{c}\text { Total } \\
\text { concentration } \\
\text { of halogenated } \\
\text { volatile organic } \\
\text { compounds } \\
\text { (TDVOC) } \\
\text { (pg/L) }\end{array}$ & $\begin{array}{c}\text { Number } \\
\text { of volatile } \\
\text { organic } \\
\text { compounds } \\
\text { detected } \\
\text { (NUM_TDVOC) }\end{array}$ \\
\hline 59 & 171 & 864 & -- & 1,817 & 56 & 1,339 & 443 & 8,134 & 17 \\
\hline 62 & 7 & 541 & -- & 1,995 & -- & 193 & -- & 7,355 & 27 \\
\hline 63 & 643 & -- & -- & 6,948 & 262 & 1,309 & 2,412 & 12,910 & 16 \\
\hline 64 & 114 & 26 & -- & 2,945 & 25 & 57 & 474 & 5,306 & 17 \\
\hline 65 & 22 & 134 & -- & 39,112 & -- & 64 & -- & 39,941 & 16 \\
\hline 66 & 50 & 10,985 & -- & 791 & 33 & 172 & 418 & 17,137 & 18 \\
\hline 66 & 43 & 5,261 & -- & 634 & -- & 73 & 196 & 9,434 & 15 \\
\hline 71 & 205 & 5,056 & -- & 7,287 & 78 & 78 & 249 & 15,961 & 17 \\
\hline 72 & 102 & 9,369 & -- & 764 & 53 & 143 & 361 & 14,569 & 18 \\
\hline 72 & 89 & 5,688 & -- & 503 & -- & 49 & 207 & 8,979 & 14 \\
\hline 73 & 64 & 9,211 & -- & 271 & 59 & 139 & 362 & 14,027 & 18 \\
\hline 73 & 84 & 6,464 & -- & 410 & -- & 50 & 196 & 10,313 & 16 \\
\hline 74 & 116 & 11,337 & -- & 3,877 & 288 & 537 & 2,756 & 22,880 & 19 \\
\hline 74 & 174 & 4,341 & -- & 2,690 & 54 & 169 & 155 & 10,507 & 17 \\
\hline 75 & 98 & 11,150 & -- & 2,846 & 86 & 259 & 472 & 18,663 & 16 \\
\hline
\end{tabular}

Estimates of average annual precipitation for 1961-90 were developed by Daly and others (1994), with the Parameterelevation Regressions on Independent Slopes Model (PRISM). PRISM uses climatic point data and a digital elevation model (DEM) to generate gridded estimates of climatic parameters. PRISM has been used extensively to map precipitation and minimum and maximum temperature over the United States, Canada, and other countries.

Three sources of soils data were used. The first were the Soil Survey Geographic database (SSURGO) soils data, which were developed by the Natural Resources Conservation Service (NRCS) at approximately 1:24,000 scale (U.S. Department of Agriculture, 2008). The SSURGO soils data included a variable for depth to groundwater within the soil horizon, which is defined as "the shallowest depth to a wet soil horizon." This is different than the depth to groundwater mentioned earlier, which used water-table elevations to calculate depth to the groundwater in the entire unsaturated zone and not just the soil horizon. In the soils data, depth to groundwater in the soils horizon was set to the maximum value of $201 \mathrm{~cm}$ (79 inches) if the depth was greater than the thickness of the soil horizon. The SSURGO data included a soil factor called "the soil septic suitability factor," which rates the suitability of a certain soil for use by septic-tank absorption fields. The suitability factor is based on soil ratings of saturated hydraulic conductivity, depth to water table in soils layer, ponding, depth to bedrock in the soil layer, and flooding between soil depths of 610 and 1,829 $\mathrm{mm}$ (24 and 72 inches). The SSURGO data do not extend east of Vail; soils data mapped in the White River National Forest were used in the portion of the study area where SSURGO soils data were not mapped (U.S. Department of Agriculture, 1999). The SSURGO and White River National Forest soils data were checked to assure consistency between the two data sets. State Soil Geographic soils data (STATSGO), which are soils data developed at a much smaller scale (approximately 1:250,000 scale), but which cover the entire study area, also were used (U.S. Department of Agriculture, 1991). The STATSGO data were not suitable for use in raw form, so STATSGO data compiled by Schwarz and Alexander (1995) were used. The Schwarz and Alexander (1995) data included weighted averaging of many of the soil characteristics. Some SSURGO and STATSGO soil variables, such as soil hydrologic group, were categorical variables. To facilitate modeling with logistic regression, categorical variables were transformed to numerical variables. For instance, soil hydrologic group is rated in four categories from $\mathrm{A}$ through $\mathrm{D}$, with A having the highest infiltration and $\mathrm{D}$ having the smallest. Soil hydrologic group A was transformed to a numerical rating of one, and soil hydrologic group D was transformed to a numerical rating of four. 


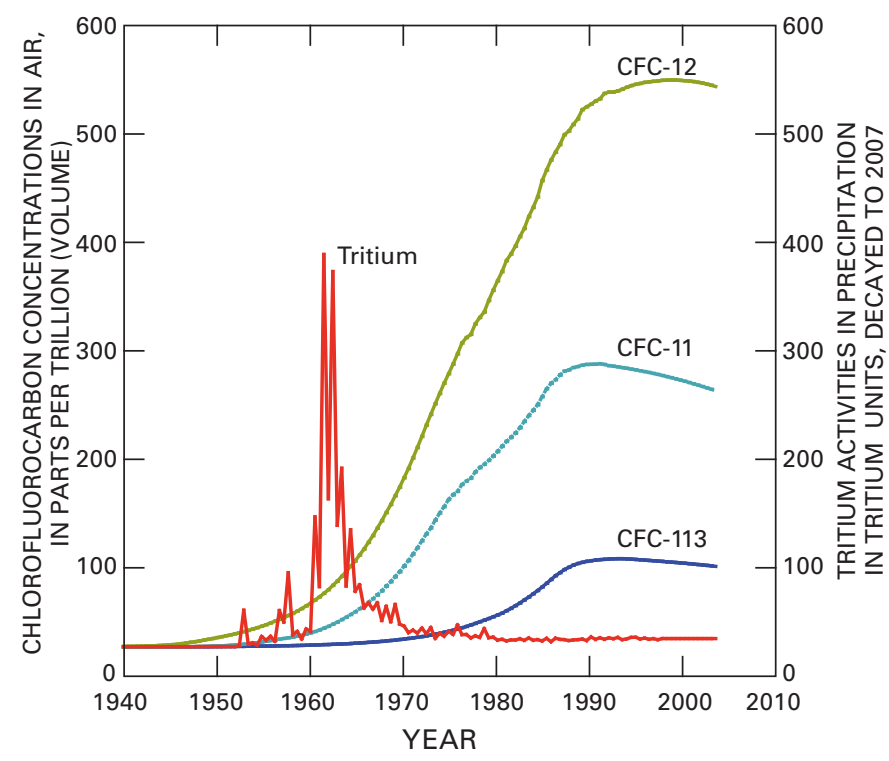

Figure 3. Concentrations of CFC-12, CFC-11, and CFC-113 in precipitation, and tritium activities in precipitation, 1940-2007.

\section{Delineating Contributing Areas to Sampled Wells}

Previous statistically based groundwater probability assessments have delineated the land cover and hydrogeologic factors potentially affecting groundwater quality by using circular buffers of various radii (Rupert, 2003). A 500-m $(1,640-\mathrm{ft})$ radius has been the most common radial length for the circular buffer method (Eckhardt and Stackelberg, 1995; Nolan and others, 2002). If the groundwater flow direction is not known, circular buffers provide a first approximation of the contributing area. However, if groundwater flow directions are known, an upgradient pie-shaped buffer may provide more accurate delineation of the contributing area (fig. 4).

Lorenz and others (2003), Gurdak (2008), Gurdak and others (2007), and Gurdak and Qi (2006) characterized land use near individual wells within an upgradient pie-shaped sector and determined that the sector method presented a more accurate estimate of land use affecting water quality in an individual well than the circular-buffer method.

A modified version of the sector method was developed and used to delineate the contributing area of wells. For each well, an upgradient 90-degree sector was established from GIS-based water-table and groundwater-flow maps. The radial length of each sector was determined based on hypothetical groundwater-flow modeling and particle-tracking simulations. MODFLOW-2000 (Harbaugh and others, 2000) was used for groundwater flow modeling, and MODPATH/MODPATH-PLOT (Pollock, 1994) was used for particle-tracking simulations of the results from the groundwater-flow modeling. Insufficient data exist for model calibration at each well location. Instead, hypothetical groundwater-flow simulations were conducted across an idealized section of the aquifer to evaluate model response to variations in boundary conditions and hydrogeologic parameters. The goal of the modeling was to determine the most probable radial length of a sector for a hypothetical well, and then apply this length to all of the wells in the study area. Because simulations were run for a hypothetical, idealized system, model calibration was not required (Reilly and Harbaugh, 2004).

To simulate regional groundwater flow, a 250-row by 250-column, two-dimensional model with square, 10-m (32.8-ft) cells was constructed. The east boundary was modeled as a river with constant head, and the west side was modeled as a drain with constant head. Regional groundwater gradients were calculated for major portions of the ERWVFA using measured water levels; the elevation of the river and the drain in the model were adjusted to simulate the average regional gradient $(12.5 \mathrm{~m} / \mathrm{km}[134.6 \mathrm{ft} / \mathrm{mi}])$. A review of drillers' records on file with the State of Colorado Engineers Office indicated that only a handful of wells drilled in the ERWVFA were drilled to the bedrock, so quantitative data on the maximum depth of the ERWVFA were not available. However, a reasonable estimate of the maximum probable thickness of the ERWVFA is $100 \mathrm{~m}$ (328 ft), so the bottom of the aquifer in the hypothetical groundwater flow model was set to $100 \mathrm{~m}$ (328 ft) below the bottom of the drain.

A forward particle-tracking method was used for all simulations. In this tracking method, one particle was started at the water table and tracked forward during an 18-year simulation. Particle-tracking estimates were calculated for an 18-year travel path because, as will be presented in the "Chlorofluorocarbons and Groundwater Age" section of this report, the median groundwater age of water sampled was 18 years. As a check

Table 5. Concentrations, approximate retention times, and minimum detection levels of halogenated volatile organic compounds detected in the Eagle River watershed, 2006-2007, Eagle County, Colorado.

[MDL, minimum detection level; pg/L, picograms per liter for sample volume of 34 cubic centimeters; na, not available; GC-ECD, gas chromatography with electron-capture detector]

\begin{tabular}{lclc}
\hline \multicolumn{1}{c}{ Compound } & $\begin{array}{c}\text { Retention } \\
\text { time } \\
\text { (minutes) }\end{array}$ & \multicolumn{1}{c}{$\begin{array}{c}\text { Alternate } \\
\text { name }\end{array}$} & $\begin{array}{c}\text { GC-ECD } \\
\text { MDL } \\
\text { (pg/L) }\end{array}$ \\
\hline dichlorodifluoromethane & 2.53 & CFC-12 & 5.5 \\
Halon 1211 & 3.36 & none & 1.3 \\
tetrafluorodichloroethane & 3.52 & CFC-114 & 22 \\
trichlorofluoromethane & 4.34 & CFC-11 & 1.2 \\
chloromethane & 4.88 & methyl chloride & 326 \\
1,1-dichloroethene & 6 & none & 72 \\
bromomethane & 6.64 & methyl bromide & 356 \\
trichlorotrifluoroethane & 7.13 & CFC-113 & 9.4 \\
methyl iodide & 9.45 & none & na \\
carbon tetrachloride & 10.17 & none & 19 \\
dichloromethane & 12.42 & methylene chloride & 564 \\
trichloromethane & 15.93 & chloroform & 52 \\
trichloroethene & 16.52 & TCE & 20 \\
tetrachloroethene & 20.35 & PCE & 9.9 \\
1,1,1-trichloroethane & 23.24 & methyl chloroform & 161 \\
\hline
\end{tabular}




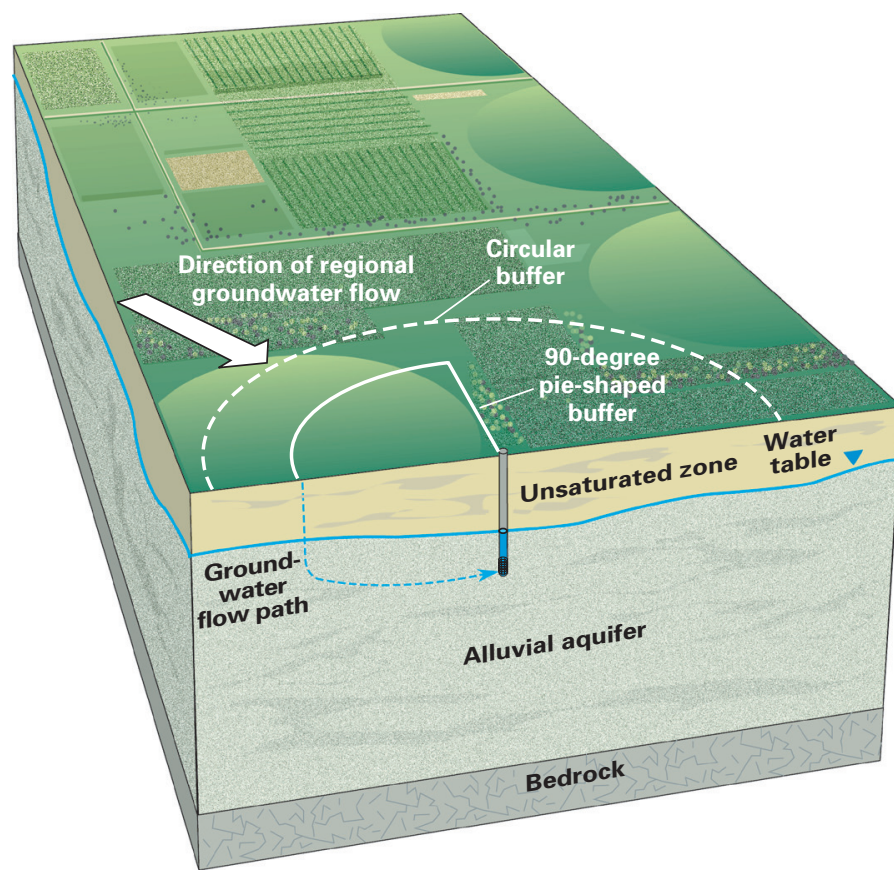

Figure 4. Conceptual diagram of 90-degree pie-shaped buffer and circular buffer for delineating areas contributing recharge to a well.

against the results of the forward-tracking simulations, backward particle-tracking simulations also were run. Because of the lack of weak or strong sinks in the simulations, backward tracking produced nearly identical results as in the forward-tracking simulations and therefore are not presented in this report.

Particles were tracked to two endpoints, the drain and a domestic well, because 72 percent of the wells sampled were domestic wells and 13 percent were monitoring wells. A hypothetical domestic well that continuously pumped water at a discharge rate of $82 \mathrm{~m}^{3} / \mathrm{d}(15 \mathrm{gal} / \mathrm{min})$ was modeled, which is conservatively large because domestic wells rarely pump continuously. Groundwater flow modeling and particle tracking indicated that there was little measurable effect on the water table or particle locations from pumping of the domestic well, indicating that groundwater quality is influenced by regional groundwater flow directions/velocities and not by induced flow paths from a single pumping domestic well. Only in the case of hydraulic conductivity $(\mathrm{K})$ values of $0.1 \mathrm{~m} / \mathrm{d}(0.3 \mathrm{ft} / \mathrm{d})$ did the domestic well have measurable effects on groundwater flow directions. The path lines of particles to the drains were used for the final determination of the radial sector length, but any location in the study area would produce results identical to the hypothetical domestic well because the model was simulating regional flow in steadystate conditions.

Very little data exist on hydraulic conductivity and groundwater recharge values in the Eagle River watershed, so a Monte Carlo approach described by Starn and others (2000) was used to determine the probability of particles reaching the drain and domestic well under a variety of hydraulic conductivity and recharge values. The term Monte Carlo refers to a computational approach where groundwater model parameters of hydraulic conductivity and recharge are randomly varied for a large number of model simulations, and the probability of a particle being in the contributing area is calculated based upon the results of the multiple simulations. Monte Carlo simulation was useful because it allowed the most probable path lengths to be bracketed.

One hundred Monte Carlo simulations were calculated using random, normally distributed values of hydraulic conductivity and recharge, which was a sufficient number of simulations to determine the most probable path lengths and remain within reasonable computational time limits. Hydraulic conductivity values were randomly varied to fit a normal distribution between 0.1 and $10 \mathrm{~m} / \mathrm{d}(0.3$ and $32.8 \mathrm{ft} / \mathrm{d})$ during the Monte Carlo simulations, which are reasonable values for unconsolidated aquifer materials consisting of various proportions of sand, silt, gravel, and clay (Bouwer, 1978). An unpublished report by an engineering firm working on municipal supply wells in the Eagle River watershed reported hydraulic conductivity values ranging from 0.1 to $6.5 \mathrm{~m} / \mathrm{d}$ $(0.3$ to $21.3 \mathrm{ft} / \mathrm{d})$ and an average value of $1.9 \mathrm{~m} / \mathrm{d}(6.2 \mathrm{ft} / \mathrm{d})$ (Todd Fessenden, Eagle River Water and Sanitation District, written commun., 2007), confirming that hydraulic conductivity values between 0.1 and $10 \mathrm{~m} / \mathrm{d}(0.3$ and $32.8 \mathrm{ft} / \mathrm{d})$ are reasonable for the Monte Carlo simulations. Daly and others (1994) reported precipitation estimates for the study area; recharge estimates based on 10 percent of the zero, minimum, median, and maximum precipitation amounts were zero, 2.8, 3.3 , and $6.6 \mathrm{~mm} / \mathrm{yr}$ respectively $(0,1.1,1.3$, and 2.6 inches/yr). During the Monte Carlo simulations, recharge estimates were randomly varied to fit a normal distribution between zero and $6.6 \mathrm{~mm} / \mathrm{d}$ ( 0 and 0.26 inches $/ \mathrm{yr}$ ) to bracket the precipitation estimates reported by Daly and others (1994). A porosity value of 0.3 was set as a constant value in the Monte Carlo simulations, which is an approximate median value for aquifer materials composed of various mixtures of silt, sand, and gravel (Bouwer, 1978).

The results of the Monte Carlo simulation indicated that there was a 0.90 probability that the path length to the drain during a 18-year time period was about $500 \mathrm{~m}$ (1,640 ft); $500 \mathrm{~m}(1,640 \mathrm{ft})$ was used in all subsequent calculations. A value of 0.90 probability was selected because this corresponds to the largest acceptable individual p-value that was used during logistic regression statistical modeling. A radial sector length of 500-m (1,640-ft) was used to determine soils properties within each pie-shaped area, which was then used in the logistic regression modeling. For each logistic regression model run, the same $500-\mathrm{m}(1,640-\mathrm{ft})$ radial sector length was used for all wells. Gurdak and Qi (2006) used different radial sector lengths depending on estimated $\mathrm{K}$ values, but data are insufficient to justify using different radial sector lengths in logistic regression model runs. 


\section{Logistic Regression Statistical Method}

Logistic regression (Hosmer and Lemeshow, 1989; Kleinbaum, 1994; Helsel and Hirsch, 1992) is a statistical method that predicts the probability of the occurrence of the target water-quality constituent above a certain level or threshold. For instance, logistic regression can predict the probability of detecting concentrations of nitrate in groundwater greater than or equal to $1 \mathrm{mg} / \mathrm{L}$. A major advantage of logistic regression over multiple regression is that the former is well suited for analysis of data sets with a large number of nondetections. Logistic regression is conceptually similar to multiple linear regression because relations between one dependent variable and several independent variables are evaluated. Whereas multiple linear regression returns a continuous value for the dependent variable, logistic regression returns the probability of a positive binomial outcome (in this case, nitrate concentrations were or were not above $1 \mathrm{mg} / \mathrm{L}$ ) in the form:

$$
P=e^{x} /\left(1+e^{x}\right)
$$

where

$$
\begin{array}{cl}
P & \text { is the probability of nitrate concentrations } \\
\text { above } 1 \mathrm{mg} / \mathrm{L} ; \\
x & \text { is } \beta_{0}+\beta_{1} \mathrm{x}_{1}+\beta_{2} \mathrm{x}_{2}+\ldots .+\beta_{\mathrm{i}} \mathrm{x}_{\mathrm{i}} \\
\beta_{\mathrm{i}} & \text { is logistic regression coefficients; } \\
\mathrm{x}_{\mathrm{i}} & \text { is values for the independent variables such as } \\
& \text { soil drainage or depth to groundwater; }
\end{array}
$$

and

$$
i \quad \text { is the number of variables. }
$$

Logistic regression calculates several statistical parameters that determine the predictive success of the model. The log-likelihood ratio measures the success of the model as a whole by comparing observed values with predicted values (Hosmer and Lemeshow, 1989, p. 13); specifically, the ratio tests whether model coefficients of the entire model are significantly different from zero. The most significant model is the one with the highest log-likelihood ratio, taking into account the number of independent variables (degrees of freedom) used in the model. The log-likelihood ratio follows a chi-squared distribution, and the computed p-value indicates whether model coefficients are significantly different from zero. Therefore, the computed $\mathrm{p}$-value is the significance level attained by the data; the smallest $p$-value indicates the best model. A p-value of 0.05 indicates a probability of a correct decision (regarding null hypothesis rejection) of 0.95 ; a p-value of 0.01 indicates a probability of 0.99 . McFadden's rho-squared (SYSTAT Software, Inc., 2004) is a transformation of the log-likelihood statistic and is intended to mimic the r-squared of linear regression. Rho-squared is always between zero and 1; a rho-squared approaching 1 corresponds to more significant results. Rho-squared tends to be smaller than r-squared, so a small number does not necessarily imply a poor fit. Values between 0.20 and 0.40 indicate good results (SYSTAT Software, Inc., 2004). P-values are calculated for each independent variable, which indicate the statistical significance that each variable has on the overall logistic regression model. Independent variables were excluded from the models if their individual $p$-values were greater than 0.1 . The sensitivity is calculated as the number of correctly predicted events (nitrate detections) divided by the total number of observed events (SYSTAT Software, Inc., 2004). The specificity is calculated as the number of correctly predicted reference events (no nitrate detections) divided by the total number of observed reference events. The total correct predictions is calculated as the number of correctly predicted events plus the number of correctly predicted reference events divided by the total number of all events. To verify the models, the percentage of actual detections was plotted with the predicted probability of detections by using a deciles of risk calculation, which typically involves partitioning the observations into 10 groups (SYSTAT Software, Inc., 2004, p. II-238).

During construction of the logistic regression models, all possible combinations of independent variables were evaluated to develop the most accurate logistic regression models. The models were built by including each individual variable in the model, evaluating the resulting test statistics, and deciding whether to include or reject the variable. Model validity and accuracy were determined by evaluating the log-likelihood ratio, McFadden's rho-squared, the model sensitivity and specificity, and the p-values calculated for each independent variable. During construction of the logistic regression models, the data were checked for multicolinearity by calculating the variance inflation factor (VIF). Multicolinearity means that at least one independent variable is closely related to one or more other independent variables. All VIFs in the final models were less than 10, which is the level of concern indicated by Helsel and Hirsch (1992, p. 306).

\section{Construction of the Probability Maps}

Maps showing the probability of groundwater contamination by various contaminants, or the likelihood of the presence of young water, were developed using the logistic regression models. Before constructing the maps, all GIS data were converted to grids with $10-\mathrm{m}$ (32.8-ft) spacing. The soils data required an additional processing step. To create the data set for logistic regression modeling, soils data were averaged within 500-m (1,640-ft) pie-shaped buffers oriented upgradient from each well site. To create the final probability maps, the soils data within 500-m (1,640-ft) pie-shaped buffers oriented upgradient from every grid cell in each soils layer/factor were averaged. After the soils data were averaged, then the logistic regression models similar to equation 1 were entered into a GIS, and a probability rating was calculated for each grid node in the study area. 
The groundwater probability models were developed as follows: (1) The groundwater quality and groundwater age data were overlaid with anthropogenic and hydrogeologic data by using a geographic information system to produce a data set in which each well had corresponding data on depth to groundwater, distance to major streams and canals, distance to gypsum beds, precipitation, soils, and well depth. These data then were downloaded to a statistical software package for analysis by logistic regression. (2) Statistical models predicting the probability of elevated nitrate concentrations, the probability of unmixed young water (using chlorofluorocarbon-11 concentrations and tritium activities), and the probability of elevated volatile organic compound concentrations were developed using logistic regression techniques. (3) The statistical models were entered into a GIS and the probability maps were constructed.

\section{Water Chemistry and Groundwater Age}

This section describes the results of water-quality sampling from sites completed in the ERWVFA and from Brush Creek, Gore Creek, Gypsum Creek, and the Eagle River. Groundwater and surface-water samples were analyzed for major ions, nutrients, ${ }^{2} \mathrm{H}$ and ${ }^{18} \mathrm{O}$ of water, tritium, CFCs, and VOCs. The results of the laboratory analyses are presented in this section; results then are synthesized in a later section entitled "Groundwater Probability Modeling."

\section{Quality Assurance and Quality Control}

The field blank, replicate, and cation-anion balance data indicate that the major-ion and nutrient data are of high quality and suitable for quantitative analysis of ground- and surfacewater quality. Concentrations of major ions and nutrients in the field blanks are mostly below laboratory reporting levels (table 6). Calcium, magnesium, nitrite, ammonia, boron, iron, and manganese were detected in a few field blanks, but in general, the concentrations were much smaller than those compounds detected in native and replicate samples (table 2, table 7). Analysis of the major-ion and nutrient field blanks indicated that the decontamination procedures of the sampling equipment were effective at cleaning the equipment between sampling sites and that there was no contamination occurring during sample collection, transport, and analysis that could affect the results of this report.

Concentrations of major ions and nutrients detected in native samples were comparable to those detected in the replicate samples (table 7). The relative percent difference (RPD) between native and replicate analyses was calculated using the following equation:

$$
\begin{gathered}
\mathrm{RPD}=\left[(\text { larger_result }- \text { smaller_result })^{*} 100\right] / \\
{[(\text { larger_result }+ \text { smaller_result }) / 2] .}
\end{gathered}
$$

The RPD of concentrations was very small for most analytes; the median RPD was 0.95 percent, the mean RPD was 3.4 percent, and the standard deviation was 7.8 percent. Laboratory alkalinity, orthophosphate, iron, and manganese had relatively large RPDs in a few samples (table 7). Laboratory alkalinity can sometimes display large RPDs because of the time lag from when the samples were collected to when they were analyzed in the laboratory. Overall, the replicate samples indicated that there was low variability (high precision) in the major-ion and nutrient analyses.

The accuracy of the major-ion and nutrient analyses also was checked by calculating the cation-anion balance. The difference between the sum of the cations and the sum of the anions (in milliequivalents) should be plus or minus 5 percent for the laboratory analyses to be considered "in control." The mean cation-anion balance for all groundwater and surfacewater native samples was 0.218 percent, the median was 0.450 percent, and the standard deviation was 1.814 percent. Out of 89 total analyses, only 2 analyses were larger than plus or minus 5 percent (5.02 and 6.45 percent). The cationanion balance indicated no discrepancies with major-ion or nutrient analyses.

\section{Major lons}

A trilinear diagram was used to represent the major-ion chemistry of surface-water samples collected from Brush Creek near Eagle, Eagle River at Avon, Eagle River at Gypsum, Eagle River at Wolcott, Gore Creek below Black Gore Creek, and Gypsum Creek near Gypsum (fig. 5). Trilinear diagrams are useful for classifying different water types and for identifying the mixing and evolution of different water types (Freeze and Cherry, 1979; Piper, 1944). A trilinear diagram shows the relative proportion of ions in a water sample, not their concentrations. The seasonal differences of surface-water chemistry in the Eagle River watershed is demonstrated by the arrows in figure 5. Surface-water samples were collected quarterly from the six surface-water sites during March, May, August, and December 2007. The samples collected during May contain a large proportion of snowmelt runoff. The August, December, and March samples from each site tend to group in a different area of figure 5 because they contain a larger proportion of base flow, which is the portion of streamflow that predominantly comes from groundwater and not snowmelt runoff. The arrows in figure 5 show the relative differences of water quality beginning in May and progressing through March of the next year.

Using a trilinear diagram, groundwaters in the ERWVFA can be classified into two major groups: groundwater that was recharged by infiltration of surface water and groundwater that had less immediate recharge from surface water and has elevated sulfate concentrations (fig. 6). Groundwater that was recharged by infiltration of surface water plots in the same vicinity as the surface-water samples (fig. 6). In many cases, these groundwaters have compositions within the seasonal fluctuations of 
Table 6. Major ion and nutrient analyses of blank samples collected from groundwater and surface water, Eagle River watershed, 2006-2007, Eagle County, Colorado, arranged chronologically.

[mg/L, milligrams per liter; $\mu \mathrm{g} / \mathrm{L}$, micrograms per liter; --, no data; $\mathrm{m}$, month; $\mathrm{d}$, day; $\mathrm{y}$, year; <, less than; e, constituent detected, but concentration is estimated]

\begin{tabular}{|c|c|c|c|c|c|c|c|c|}
\hline Local identification number & 74 & 32 & 60 & 73 & 5 & 42 & 40 & 72 \\
\hline U.S. Geological Survey station identification number & 09069000 & 394040106455701 & 393658106574101 & 09069500 & 393743106171000 & 393813106490201 & 393758106473601 & 09068000 \\
\hline Date of sample collection (mmddyy) & $02 / 13 / 07$ & $05 / 15 / 07$ & $05 / 20 / 07$ & $05 / 24 / 07$ & 06/02/07 & 06/10/07 & 06/23/07 & 08/30/07 \\
\hline Alkalinity, dissolved, $\mathrm{mg} / \mathrm{L}$ as calcium carbonate & -- & $<5$ & $<5$ & $<5$ & $<5$ & $<5$ & $<5$ & $<5$ \\
\hline Ammonia, dissolved, $\mathrm{mg} / \mathrm{L}$ as nitrogen & $<0.020$ & $<0.020$ & $\mathrm{e} 0.012$ & -- & $\mathrm{e} 0.010$ & $<0.020$ & $<0.020$ & -- \\
\hline Nitrite, dissolved, $\mathrm{mg} / \mathrm{L}$ as nitrogen & $<0.002$ & $<0.002$ & $<0.002$ & -- & $\mathrm{e} 0.001$ & $<0.002$ & $<0.002$ & -- \\
\hline Nitrite plus nitrate, dissolved, $\mathrm{mg} / \mathrm{L}$ as nitrogen & $<0.016$ & $<0.06$ & $<0.06$ & -- & $<0.06$ & $<0.06$ & $<0.06$ & -- \\
\hline Orthophosphate, dissolved, $\mathrm{mg} / \mathrm{L}$ as phosphorus & $<0.006$ & $<0.006$ & $<0.006$ & -- & $<0.006$ & $<0.006$ & $<0.006$ & -- \\
\hline Calcium, dissolved, $\mathrm{mg} / \mathrm{L}$ & $<0.02$ & $<0.02$ & $<0.02$ & $<0.02$ & 0.14 & 0.12 & 0.03 & $<0.02$ \\
\hline Magnesium, dissolved, mg/L & $<0.014$ & $<0.014$ & $<0.014$ & $<0.014$ & $<0.014$ & $\mathrm{e} 0.008$ & $<0.014$ & $<0.014$ \\
\hline Sodium, dissolved, mg/L & $<0.20$ & $<0.20$ & $<0.20$ & $<0.20$ & $<0.20$ & $<0.20$ & $<0.20$ & $<0.20$ \\
\hline Potassium, dissolved, mg/L & $<0.04$ & $<0.04$ & $<0.04$ & $<0.04$ & $<0.04$ & $<0.04$ & $<0.04$ & $<0.04$ \\
\hline Chloride, dissolved, $\mathrm{mg} / \mathrm{L}$ & $<0.12$ & $<0.12$ & $<0.12$ & $<0.12$ & $<0.12$ & $<0.12$ & $<0.12$ & $<0.12$ \\
\hline Sulfate, dissolved, $\mathrm{mg} / \mathrm{L}$ & $<0.18$ & $<0.18$ & $<0.18$ & $<0.18$ & $<0.18$ & $<0.18$ & $<0.18$ & $<0.18$ \\
\hline Fluoride, dissolved, $\mathrm{mg} / \mathrm{L}$ & $<0.10$ & $<0.10$ & $<0.10$ & $<0.10$ & $<0.10$ & $<0.10$ & $<0.10$ & $<0.10$ \\
\hline Silica, dissolved, $\mathrm{mg} / \mathrm{L}$ & $<0.2$ & $<0.2$ & $<0.2$ & $<0.2$ & $<0.2$ & $<0.2$ & $<0.2$ & $<0.2$ \\
\hline Boron, dissolved, $\mu \mathrm{g} / \mathrm{L}$ & -- & $<1.8$ & 2.2 & e1.6 & 1.9 & 2.6 & 2.4 & $<1.8$ \\
\hline Iron, dissolved, $\mu \mathrm{g} / \mathrm{L}$ & -- & $<6$ & $<6$ & $<6$ & 11 & e3 & $<6$ & $<6$ \\
\hline Manganese, dissolved, $\mu \mathrm{g} / \mathrm{L}$ & -- & $<0.2$ & $<0.2$ & $<0.2$ & 0.3 & $\mathrm{e} 0.2$ & $<0.2$ & $<0.2$ \\
\hline
\end{tabular}


Table 7. Analyses of replicate samples of major ions, nutrients, stable isotopes of hydrogen and oxygen, and tritium collected from groundwater and surface water, Eagle River watershed, 2006-2007, Eagle County, Colorado, arranged chronologically.

$\left[\mathrm{CaCO}_{3}\right.$, calcium carbonate; ${ }^{\circ} \mathrm{C}$, degrees Celsius; \%, percent; diss., dissolved; lab., laboratory analysis; $\mu \mathrm{S} / \mathrm{cm}$, microsiemens per centimeter; mg/L, milligrams per liter; $\mu \mathrm{g} / \mathrm{L}$, micrograms per liter; $\mathrm{N}$, nitrogen; P, phosphorus; pCi/L, picocuries per liter; RPD, relative percent difference; --, no data; m, month; d, day; y, year; < less than; e, estimated]

\begin{tabular}{|c|c|c|c|c|c|c|c|c|c|c|c|c|}
\hline $\begin{array}{c}\text { Local identification number } \\
\begin{array}{l}\text { U.S. Geological Survey station identification number } \\
\text { Date of sample collection (mmddyy) }\end{array}\end{array}$ & & $\begin{array}{l}11 \\
30210624350 \\
08 / 24 / 06\end{array}$ & & \multicolumn{2}{|r|}{09066510} & & & $\begin{array}{l}66 \\
73710616590 \\
12 / 20 / 06\end{array}$ & & \multicolumn{3}{|c|}{$\begin{array}{c}09068000 \\
03 / 20 / 07\end{array}$} \\
\hline Sample type & Native & Replicate & RPD, \% & Native & Replicate & RPD, \% & Native & Replicate & RPD, \% & Native & Replicate & RPD, \% \\
\hline Specific conductance, lab., $\mu \mathrm{S} / \mathrm{cm}$ at $25^{\circ} \mathrm{C}$ & 637 & 637 & 0.0 & -- & -- & -- & 230 & 229 & 0.4 & 469 & 468 & 0.2 \\
\hline $\mathrm{pH}$, lab., standard units & 7.5 & 7.5 & 0.0 & -- & -- & -- & 8.2 & 8.2 & 0.0 & 8.3 & 8.3 & 0.0 \\
\hline Alkalinity, diss., lab., $\mathrm{mg} / \mathrm{L}$ as $\mathrm{CaCO}_{3}$ & 181 & 181 & 0.0 & -- & -- & -- & 68 & 69 & 1.5 & 111 & 110 & 0.9 \\
\hline Alkalinity, diss., field, $\mathrm{mg} / \mathrm{L}$ as $\mathrm{CaCO}_{3}$ & 177 & 175 & 1.1 & 111 & 112 & 0.9 & 66 & 67 & 1.5 & 82 & 102 & 21.7 \\
\hline Ammonia, diss., $\mathrm{mg} / \mathrm{L}$ as $\mathrm{N}$ & 0.01 & $\mathrm{e} 0.009$ & -- & $\mathrm{e} 0.015$ & $\mathrm{e} 0.013$ & -- & -- & -- & -- & -- & -- & -- \\
\hline Nitrite, diss., $\mathrm{mg} / \mathrm{L}$ as $\mathrm{N}$ & $<0.002$ & $<0.002$ & -- & 0.03 & 0.03 & 0.0 & -- & -- & -- & -- & -- & -- \\
\hline Nitrite plus nitrate, diss., $\mathrm{mg} / \mathrm{L}$ as $\mathrm{N}$ & 0.99 & 0.99 & 0.0 & 1.38 & 1.32 & 4.4 & -- & -- & -- & -- & -- & -- \\
\hline Orthophosphate, diss., $\mathrm{mg} / \mathrm{L}$ as $\mathrm{P}$ & 0.011 & 0.01 & 9.5 & 0.154 & 0.153 & 0.7 & -- & -- & -- & -- & -- & -- \\
\hline Calcium, diss., $\mathrm{mg} / \mathrm{L}$ & 91.3 & 89.6 & 1.9 & 51 & 52.3 & 2.5 & 23.7 & 24.5 & 3.3 & 69.8 & 68.7 & 1.6 \\
\hline Magnesium, diss., $\mathrm{mg} / \mathrm{L}$ & 18.2 & 17.9 & 1.7 & 9.4 & 9.63 & 2.4 & 4.65 & 4.79 & 3.0 & 13.6 & 13.8 & 1.5 \\
\hline Sodium, diss., mg/L & 9.25 & 9.09 & 1.7 & 10.8 & 11.1 & 2.7 & 11.9 & 12.3 & 3.3 & 3.12 & 3.11 & 0.3 \\
\hline Potassium, diss., $\mathrm{mg} / \mathrm{L}$ & 1.53 & 1.49 & 2.6 & 1.55 & 1.57 & 1.3 & 0.59 & 0.61 & 3.3 & 0.71 & 0.72 & 1.4 \\
\hline Chloride, diss., mg/L & 33.7 & 33.6 & 0.3 & 23.3 & 23.5 & 0.9 & 25.6 & 25.8 & 0.8 & 1.1 & 1.08 & 1.8 \\
\hline Sulfate, diss., $\mathrm{mg} / \mathrm{L}$ & 101 & 100 & 1.0 & 45 & 45.3 & 0.7 & 3.31 & 3.3 & 0.3 & 126 & 125 & 0.8 \\
\hline Fluoride, diss., mg/L & 0.13 & 0.12 & 8.0 & 0.13 & 0.13 & 0.0 & e0.09 & $\mathrm{e} 0.08$ & -- & $\mathrm{e} 0.05$ & $\mathrm{e} 0.06$ & -- \\
\hline Silica, diss., mg/L & 7.8 & 7.8 & 0.0 & 4.85 & 4.97 & 2.4 & 5.4 & 5.4 & 0.0 & 9.1 & 9 & 1.1 \\
\hline Boron, diss., $\mu \mathrm{g} / \mathrm{L}$ & 26 & 27 & 3.8 & -- & -- & -- & 6.3 & 6.7 & 6.2 & 10 & 11 & 9.5 \\
\hline Iron, diss., $\mu \mathrm{g} / \mathrm{L}$ & 7 & e3 & -- & -- & -- & -- & 13 & 18 & 32.3 & 10 & 9 & 10.5 \\
\hline Manganese, diss., $\mu \mathrm{g} / \mathrm{L}$ & $<0.6$ & $<0.6$ & -- & 0.8 & 0.8 & 0.0 & 12.1 & 12.2 & 0.8 & 5.5 & 5.3 & 3.7 \\
\hline Hydrogen $2 / 1$ ratio, per mil & -128 & -128 & 0.0 & -- & -- & -- & -126 & -125 & 0.8 & -130 & -129 & 0.8 \\
\hline Oxygen $18 / 16$ ratio, per mil & -17.33 & -17.35 & 0.1 & -- & -- & -- & -17.27 & -17.22 & 0.3 & -17.48 & -17.45 & 0.2 \\
\hline Tritium, $\mathrm{pCi} / \mathrm{L}$ & 34 & 32 & 6.1 & -- & -- & -- & 27.2 & 28.8 & 5.7 & 30.72 & 30.72 & 0.0 \\
\hline Tritium 2-sigma combined uncertainty, $\mathrm{pCi} / \mathrm{L}$ & 1.3 & 1.3 & 0.0 & -- & -- & -- & 0.96 & 0.96 & 0.0 & 0.96 & 0.96 & 0.0 \\
\hline
\end{tabular}


Table 7. Analyses of replicate samples of major ions, nutrients, stable isotopes of hydrogen and oxygen, and tritium collected from groundwater and surface water, Eagle River watershed, 2006-2007, Eagle County, Colorado, arranged chronologically.-Continued

$\left[\mathrm{CaCO}_{3}\right.$, calcium carbonate; ${ }^{\circ} \mathrm{C}$, degrees Celsius; \%, percent; diss., dissolved; lab., laboratory analysis; $\mu \mathrm{S} / \mathrm{cm}$, microsiemens per centimeter; $\mathrm{mg} / \mathrm{L}$, milligrams per liter; $\mu \mathrm{g} / \mathrm{L}$, micrograms per liter; $\mathrm{N}$, nitrogen; P, phosphorus; pCi/L, picocuries per liter; RPD, relative percent difference; --, no data; m, month; d, day; y, year; < less than; e, estimated]

\begin{tabular}{|c|c|c|c|c|c|c|c|c|c|c|c|c|}
\hline $\begin{array}{c}\text { Local identification number } \\
\begin{array}{c}\text { U.S. Geological Survey station identification number } \\
\text { Date of sample collection (mmddyy) }\end{array}\end{array}$ & & $\begin{array}{c}70 \\
09067005 \\
04 / 18 / 07 \\
\end{array}$ & & & $\begin{array}{c}32 \\
10401064557 \\
05 / 15 / 07 \\
\end{array}$ & & & $\begin{array}{c}60 \\
6581065741 \\
5 / 20 / 2007\end{array}$ & & & $\begin{array}{c}8 \\
8301062106 \\
06 / 05 / 07 \\
\end{array}$ & \\
\hline Sample type & Native & Replicate & RPD, \% & Native & Replicate & RPD, \% & Native & Replicate & RPD, \% & Native & Replicate & RPD, \% \\
\hline Specific conductance, lab., $\mu \mathrm{S} / \mathrm{cm}$ at $25^{\circ} \mathrm{C}$ & -- & -- & -- & 848 & 840 & 0.9 & 564 & 575 & 1.9 & 269 & 269 & 0.0 \\
\hline pH, lab., standard units & -- & -- & -- & 7.8 & 7.8 & 0.0 & 7.9 & 8 & 1.3 & 8.3 & 8.3 & 0.0 \\
\hline Alkalinity, diss., lab., $\mathrm{mg} / \mathrm{L}$ as $\mathrm{CaCO}_{3}$ & -- & -- & -- & 204 & 173 & 16.4 & 143 & 175 & 20.1 & 114 & 114 & 0.0 \\
\hline Alkalinity, diss., field, $\mathrm{mg} / \mathrm{L}$ as $\mathrm{CaCO}_{3}$ & 72 & 72 & 0.0 & 224 & 224 & 0.0 & 199 & 188 & 5.7 & 115 & 113 & 1.8 \\
\hline Ammonia, diss., $\mathrm{mg} / \mathrm{L}$ as $\mathrm{N}$ & $<0.020$ & $<0.020$ & -- & $<0.020$ & $<0.020$ & -- & $<0.020$ & $<0.020$ & -- & $<0.020$ & $<0.020$ & -- \\
\hline Nitrite, diss., $\mathrm{mg} / \mathrm{L}$ as $\mathrm{N}$ & $\mathrm{e} 0.001$ & $\mathrm{e} 0.001$ & -- & $<0.002$ & $<0.002$ & -- & $<0.002$ & $<0.002$ & -- & $<0.002$ & $<0.002$ & -- \\
\hline Nitrite plus nitrate, diss., $\mathrm{mg} / \mathrm{L}$ as $\mathrm{N}$ & 0.164 & 0.166 & 1.2 & 1.43 & 1.42 & 0.7 & 0.26 & 0.27 & 3.8 & 0.23 & 0.24 & 4.3 \\
\hline Orthophosphate, diss., $\mathrm{mg} / \mathrm{L}$ as $\mathrm{P}$ & $\mathrm{e} 0.006$ & $\mathrm{e} 0.006$ & -- & 0.007 & 0.007 & 0.0 & 0.006 & 0.008 & 28.6 & $<0.006$ & $<0.006$ & -- \\
\hline Calcium, diss., $\mathrm{mg} / \mathrm{L}$ & 28.9 & 28.7 & 0.7 & 110 & 108 & 1.8 & 88 & 86.9 & 1.3 & 39.5 & 39.2 & 0.8 \\
\hline Magnesium, diss., $\mathrm{mg} / \mathrm{L}$ & 7.95 & 8.11 & 2.0 & 24 & 24 & 0.0 & 22.5 & 22.2 & 1.3 & 6.11 & 6.07 & 0.7 \\
\hline Sodium, diss., mg/L & 6.19 & 6.13 & 1.0 & 44.8 & 44.4 & 0.9 & 4.45 & 4.42 & 0.7 & 5.54 & 5.47 & 1.3 \\
\hline Potassium, diss., mg/L & 0.88 & 0.88 & 0.0 & 3.28 & 3.32 & 1.2 & 1.22 & 1.19 & 2.5 & 1.3 & 1.3 & 0.0 \\
\hline Chloride, diss., mg/L & 10.1 & 10 & 1.0 & 53.6 & 53.6 & 0.0 & 1.37 & 1.35 & 1.5 & 13.1 & 13 & 0.8 \\
\hline Sulfate, diss., mg/L & 28.4 & 28.3 & 0.4 & 140 & 140 & 0.0 & 111 & 111 & 0.0 & 5.07 & 5.17 & 2.0 \\
\hline Fluoride, diss., mg/L & 0.1 & 0.1 & 0.0 & 0.17 & 0.16 & 6.1 & 0.12 & 0.11 & 8.7 & 0.12 & 0.11 & 8.7 \\
\hline Silica, diss., mg/L & 6.11 & 6.09 & 0.3 & 15.1 & 15.1 & 0.0 & 13.6 & 13.6 & 0.0 & 6.8 & 6.8 & 0.0 \\
\hline Boron, diss., $\mu \mathrm{g} / \mathrm{L}$ & -- & -- & -- & 39 & 38 & 2.6 & 21 & 20 & 4.9 & 6.7 & 6.9 & 2.9 \\
\hline Iron, diss., $\mu \mathrm{g} / \mathrm{L}$ & -- & -- & -- & 8 & 7 & 13.3 & 65 & 59 & 9.7 & $<6$ & $<6$ & -- \\
\hline Manganese, diss., $\mu \mathrm{g} / \mathrm{L}$ & 66.6 & 66.5 & 0.2 & 0.5 & 0.3 & 50.0 & 1.7 & 1.5 & 12.5 & 12.2 & 13.7 & 11.6 \\
\hline Hydrogen $2 / 1$ ratio, per mil & -- & -- & -- & -126 & -126 & 0.0 & -125 & -124 & 0.8 & -126 & -126 & 0.0 \\
\hline Oxygen $18 / 16$ ratio, per mil & -- & -- & -- & -16.92 & -16.98 & 0.4 & -17.09 & -17.06 & 0.2 & -17.23 & -17.08 & 0.9 \\
\hline Tritium, $\mathrm{pCi} / \mathrm{L}$ & -- & -- & -- & 25.92 & 26.56 & 2.4 & 29.76 & 31.04 & 4.2 & 28.16 & 27.52 & 2.3 \\
\hline Tritium 2-sigma combined uncertainty, $\mathrm{pCi} / \mathrm{L}$ & -- & -- & -- & 0.96 & 0.96 & 0.0 & 0.96 & 0.96 & 0.0 & 0.96 & 0.96 & 0.0 \\
\hline
\end{tabular}


Table 7. Analyses of replicate samples of major ions, nutrients, stable isotopes of hydrogen and oxygen, and tritium collected from groundwater and surface water, Eagle River watershed, 2006-2007, Eagle County, Colorado, arranged chronologically.-Continued

$\left[\mathrm{CaCO}_{3}\right.$, calcium carbonate; ${ }^{\circ} \mathrm{C}$, degrees Celsius; \%, percent; diss., dissolved; lab., laboratory analysis; $\mu \mathrm{S} / \mathrm{cm}$, microsiemens per centimeter; mg/L, milligrams per liter; $\mu \mathrm{g} / \mathrm{L}$, micrograms per liter; $\mathrm{N}$, nitrogen; P, phosphorus; $\mathrm{pCi} / \mathrm{L}$, picocuries per liter; RPD, relative percent difference; --, no data; m, month; d, day; y, year; < less than; e, estimated]

\begin{tabular}{|c|c|c|c|c|c|c|c|c|c|c|c|c|c|c|c|}
\hline \multirow{2}{*}{$\begin{array}{l}\text { Local identification number } \\
\begin{array}{l}\text { U.S. Geological Survey station identification number } \\
\text { Date of sample collection (mmddyy) }\end{array} \\
\text { Sample type }\end{array}$} & & $\begin{array}{c}42 \\
381310649020 \\
06 / 10 / 07\end{array}$ & & & $\begin{array}{c}40 \\
37581064736 \\
06 / 20 / 07 \\
\end{array}$ & & \multicolumn{3}{|c|}{$\begin{array}{c}394220106431500 \\
07 / 27 / 07\end{array}$} & \multicolumn{3}{|c|}{$\begin{array}{c}09069500 \\
08 / 31 / 07\end{array}$} & \multicolumn{3}{|c|}{$\begin{array}{l}09069000 \\
09 / 12 / 06\end{array}$} \\
\hline & Native & Replicate & RPD, \% & Native & Replicate & RPD, \% & Native & Replicate & RPD, \% & Native & Replicate & RPD, \% & Native & Replicate & RPD, $\%$ \\
\hline Specific conductance, lab., $\mu \mathrm{S} / \mathrm{cm}$ at $25^{\circ} \mathrm{C}$ & 778 & 747 & 4.1 & 867 & 841 & 3.0 & -- & -- & -- & 427 & 428 & 0.2 & -- & -- & -- \\
\hline $\mathrm{pH}$, lab., standard units & 8 & 7.8 & 2.5 & 7.7 & 7.7 & 0.0 & -- & -- & -- & 8.3 & 8.3 & 0.0 & -- & -- & -- \\
\hline Alkalinity, diss., lab., $\mathrm{mg} / \mathrm{L}$ as $\mathrm{CaCO}_{3}$ & 158 & 157 & 0.6 & 163 & 176 & 7.7 & -- & -- & -- & 119 & 120 & 0.8 & -- & -- & -- \\
\hline Alkalinity, diss., field, $\mathrm{mg} / \mathrm{L}$ as $\mathrm{CaCO}_{3}$ & 163 & 159 & 2.5 & 195 & 190 & 2.6 & 76 & 75 & 1.3 & 113 & 112 & 0.9 & 125 & 127 & 1.6 \\
\hline Ammonia, diss., $\mathrm{mg} / \mathrm{L}$ as $\mathrm{N}$ & $<0.020$ & $<0.020$ & -- & $<0.020$ & $<0.020$ & -- & $\mathrm{e} 0.016$ & -- & -- & -- & -- & -- & $\mathrm{e} 0.007$ & $\mathrm{e} 0.009$ & -- \\
\hline Nitrite, diss., $\mathrm{mg} / \mathrm{L}$ as $\mathrm{N}$ & $<0.002$ & $<0.002$ & -- & $<0.002$ & $<0.002$ & -- & 0.004 & -- & -- & -- & -- & -- & 0.004 & 0.004 & 0.0 \\
\hline Nitrite plus nitrate, diss., $\mathrm{mg} / \mathrm{L}$ as $\mathrm{N}$ & 0.35 & 0.33 & 5.9 & 0.36 & 0.35 & 2.8 & 0.292 & -- & -- & -- & -- & -- & 0.41 & 0.407 & 0.7 \\
\hline Orthophosphate, diss., $\mathrm{mg} / \mathrm{L}$ as $\mathrm{P}$ & 0.008 & 0.007 & 13.3 & 0.007 & 0.007 & 0.0 & 0.037 & -- & -- & -- & -- & -- & 0.038 & 0.037 & 2.7 \\
\hline Calcium, diss., $\mathrm{mg} / \mathrm{L}$ & 105 & 105 & 0.0 & 111 & 112 & 0.9 & 37 & 36.5 & 1.4 & 72.7 & 74.6 & 2.6 & 83.4 & 82.8 & 0.7 \\
\hline Magnesium, diss., $\mathrm{mg} / \mathrm{L}$ & 16.6 & 16.4 & 1.2 & 19.9 & 20.1 & 1.0 & 8.73 & 8.58 & 1.7 & 8.33 & 8.57 & 2.8 & 16.1 & 16 & 0.6 \\
\hline Sodium, diss., mg/L & 29 & 29.2 & 0.7 & 43.1 & 43.4 & 0.7 & 35.5 & 35 & 1.4 & 2.31 & 2.41 & 4.2 & 50.5 & 50 & 1.0 \\
\hline Potassium, diss., mg/L & 2.94 & 2.93 & 0.3 & 2.85 & 2.92 & 2.4 & 1.55 & 1.51 & 2.6 & 0.4 & 0.44 & 9.5 & 2.54 & 2.51 & 1.2 \\
\hline Chloride, diss., mg/L & 51.6 & 50.5 & 2.2 & 55.2 & 55 & 0.4 & 53 & 53.3 & 0.6 & 0.38 & 0.38 & 0.0 & 84.3 & 84.2 & 0.1 \\
\hline Sulfate, diss., mg/L & 158 & 155 & 1.9 & 177 & 177 & 0.0 & 50.1 & 50.5 & 0.8 & 98.1 & 98 & 0.1 & 159 & 159 & 0.0 \\
\hline Fluoride, diss., $\mathrm{mg} / \mathrm{L}$ & 0.13 & 0.14 & 7.4 & 0.13 & 0.15 & 14.3 & $\mathrm{e} 0.10$ & 0.11 & -- & $<0.10$ & $\mathrm{e} 0.06$ & -- & 0.15 & 0.18 & 18.2 \\
\hline Silica, diss., mg/L & 11.6 & 11.5 & 0.9 & 12.9 & 13 & 0.8 & 4.53 & 4.52 & 0.2 & 9 & 9.1 & 1.1 & 5.23 & 5.2 & 0.6 \\
\hline Boron, diss., $\mu \mathrm{g} / \mathrm{L}$ & 20 & 20 & 0.0 & 30 & 30 & 0.0 & -- & -- & -- & 9.6 & 9.8 & 2.1 & -- & -- & -- \\
\hline Iron, diss., $\mu \mathrm{g} / \mathrm{L}$ & 151 & 173 & 13.6 & 13 & 17 & 26.7 & -- & -- & -- & e5 & 7 & -- & -- & -- & -- \\
\hline Manganese, diss., $\mu \mathrm{g} / \mathrm{L}$ & 4.3 & 5.7 & 28.0 & 2.4 & 4.9 & 68.5 & -- & -- & -- & 2.1 & 2.1 & 0.0 & 14.3 & -- & -- \\
\hline Hydrogen $2 / 1$ ratio, per mil & -126 & -126 & 0.0 & -126 & -125 & 0.8 & -- & -- & -- & -125 & -125 & 0.0 & -- & -- & -- \\
\hline Oxygen $18 / 16$ ratio, per mil & -17.17 & -17.17 & 0.0 & -17.03 & -17.11 & 0.5 & -- & -- & -- & -17.03 & -17.03 & 0.0 & -- & -- & -- \\
\hline Tritium, $\mathrm{pCi} / \mathrm{L}$ & 26.88 & 30.72 & 13.3 & 25.6 & 27.2 & 6.1 & -- & -- & -- & 32 & 33 & 3.1 & -- & -- & -- \\
\hline Tritium 2-sigma combined uncertainty, $\mathrm{pCi} / \mathrm{L}$ & 0.96 & 0.96 & 0.0 & 0.96 & 0.96 & 0.0 & -- & -- & -- & 2.6 & 2.6 & 0.0 & -- & -- & -- \\
\hline
\end{tabular}




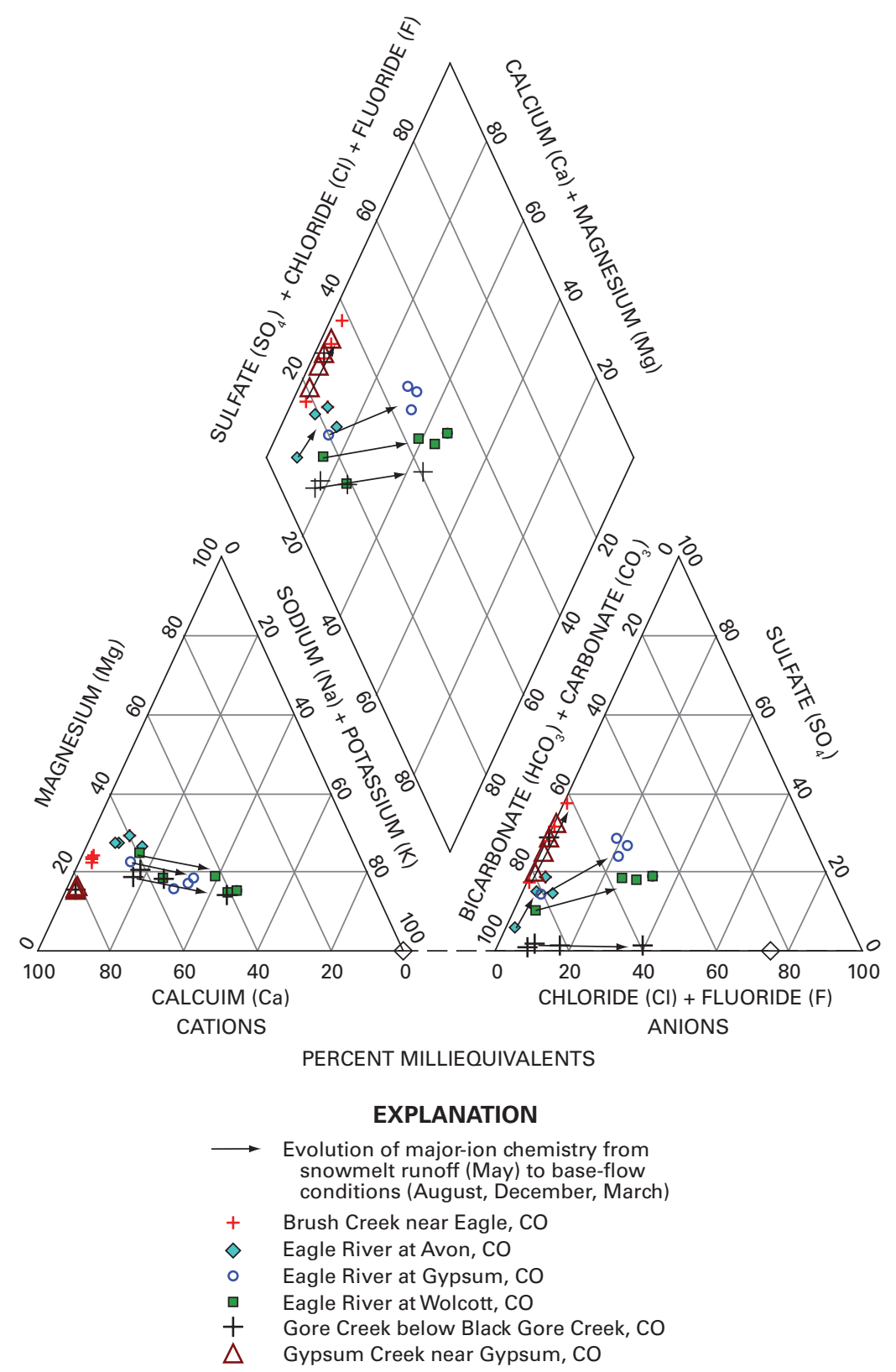

Figure 5. Major-ion chemistry of surface-water-quality samples, Eagle River watershed, 2006-2007, Eagle County, Colorado.

the surface-water chemistry (sulfate concentrations less than $750 \mathrm{mg} / \mathrm{L}$ ), and the wells that produced these groundwaters are mostly located in the eastern portion of the study area and are adjacent to creeks and rivers (fig. 7). The second category of groundwater plotted in a different portion of the trilinear diagram than the surface-water samples (fig. 6) and has sulfate concentrations greater than $750 \mathrm{mg} / \mathrm{L}$, which is three times greater than the Secondary Drinking Water Regulation of $250 \mathrm{mg} / \mathrm{L}$ (U.S. Environmental Protection Agency, 2003). The concentration of $750 \mathrm{mg} / \mathrm{L}$ was selected because it is an apparent separation point on the trilinear diagram; the $750 \mathrm{mg} / \mathrm{L}$ concentration has no hydrogeologic or health standards associated with it. The locations of wells with sulfate concentrations less than $250 \mathrm{mg} / \mathrm{L}$, greater than $250 \mathrm{mg} / \mathrm{L}$ but less than $750 \mathrm{mg} / \mathrm{L}$, and greater than $750 \mathrm{mg} / \mathrm{L}$ are shown in figure 7.

Groundwater samples from a subset of wells exceeded the USEPA National Secondary Drinking Water Regulations (U.S. Environmental Protection Agency, 2003) for chloride (250 mg/L), sulfate $(250 \mathrm{mg} / \mathrm{L})$, fluoride $(2.0 \mathrm{mg} / \mathrm{L})$, total dissolved solids (500 mg/L), iron $(300 \mu \mathrm{g} / \mathrm{L})$, and manganese $(50 \mu \mathrm{g} / \mathrm{L})($ table 2 , table 8$)$. National Secondary Drinking Water Regulations (secondary standards) are guidelines for constituents that may cause cosmetic effects (such as skin or tooth discoloration [for fluoride]) or esthetic effects (such as taste, 


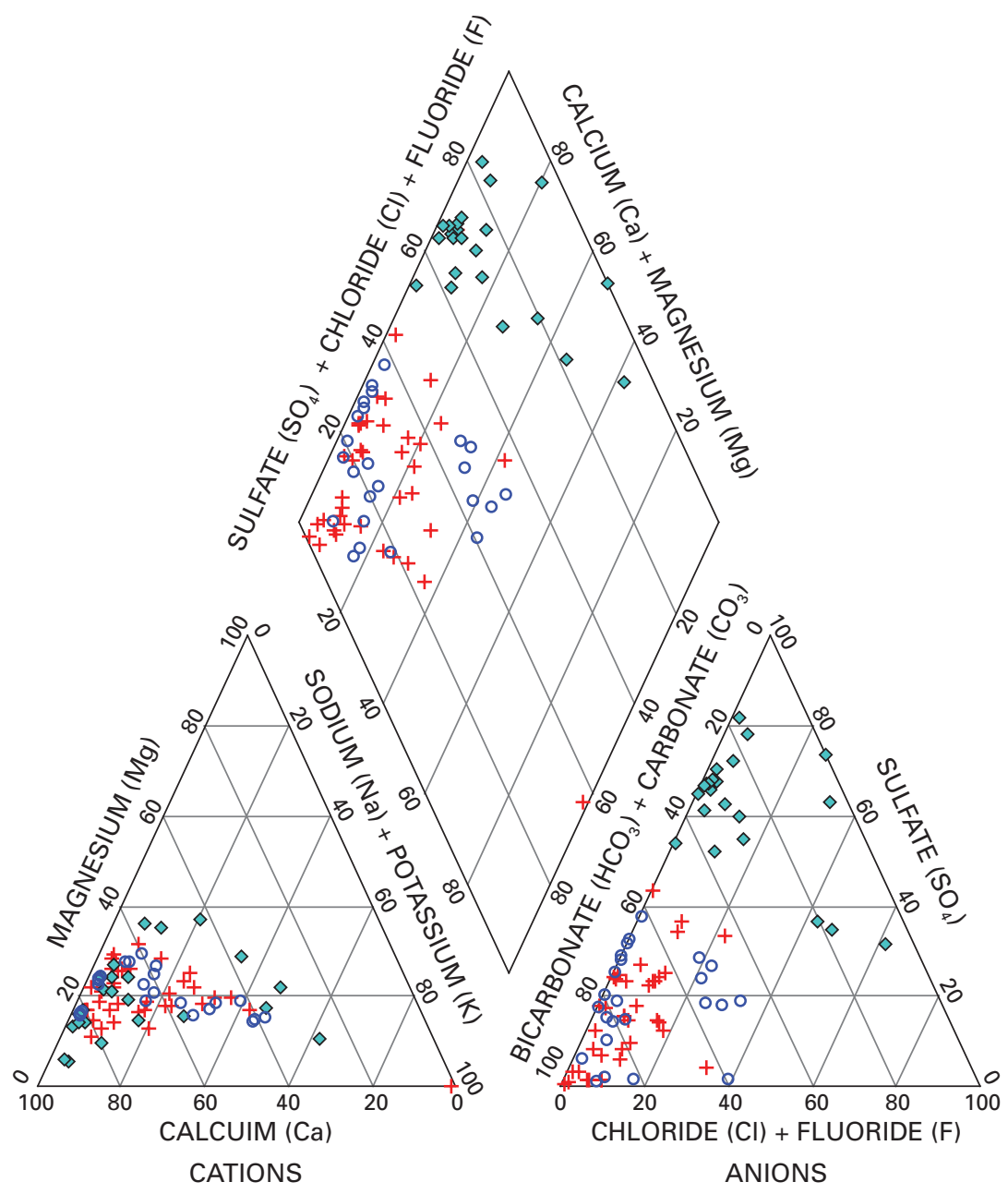

PERCENT MILLIEQUIVALENTS

\section{EXPLANATION}

$\diamond$ Groundwater with sulfate greater than $750 \mathrm{mg} / \mathrm{L}$

+ Groundwater with sulfate less than $750 \mathrm{mg} / \mathrm{L}$

- Surface water

Figure 6. Major-ion chemistry of ground- and surface-water-quality samples, Eagle River watershed, 2006-2007, Eagle County, Colorado.

odor, or color [for sulfate, total dissolved solids, iron, and manganese]) in drinking water. Exceedances for sulfate and total dissolved solids were common and were mostly located west of Wolcott (fig. 7, fig. 8). Areas where sulfate and total dissolved solids exceed the secondary drinking-water standards, such as near the town of Gypsum, probably would not be suitable for development as public drinking-water supplies because of the difficulty of lowering the sulfate and total dissolved solids concentrations below the Secondary Drinking Water Regulations. Exceedances for chloride, fluoride, iron, and manganese were not common and were located in the western portion of the ERWVFA (table 2).

Sulfate exceeded the secondary standard of $250 \mathrm{mg} / \mathrm{L}$ in many wells near Eagle, Gypsum, and Dotsero (fig. 7). Health concerns regarding sulfate in drinking water have been raised because of reports that diarrhea may be associated with the ingestion of water containing high levels of sulfate (U.S. Environmental Protection Agency, 1999). Of particular concern are groups within the general population that may be at greater risk from the laxative effects of sulfate when they experience an abrupt change from drinking water with low sulfate concentrations to drinking water with high sulfate concentrations. Many domestic well owners in the western portion of Eagle County use reverse-osmosis water-treatment systems to reduce the amount of sulfate in their drinking water. The most significant source of sulfate and total dissolved solids to groundwater in the Eagle River watershed is the Eagle Valley Evaporite, which is a Pennsylvanian-age geologic deposit located in the western half of Eagle County (fig. 7). The Eagle Valley Evaporite is composed mostly of gypsum with 


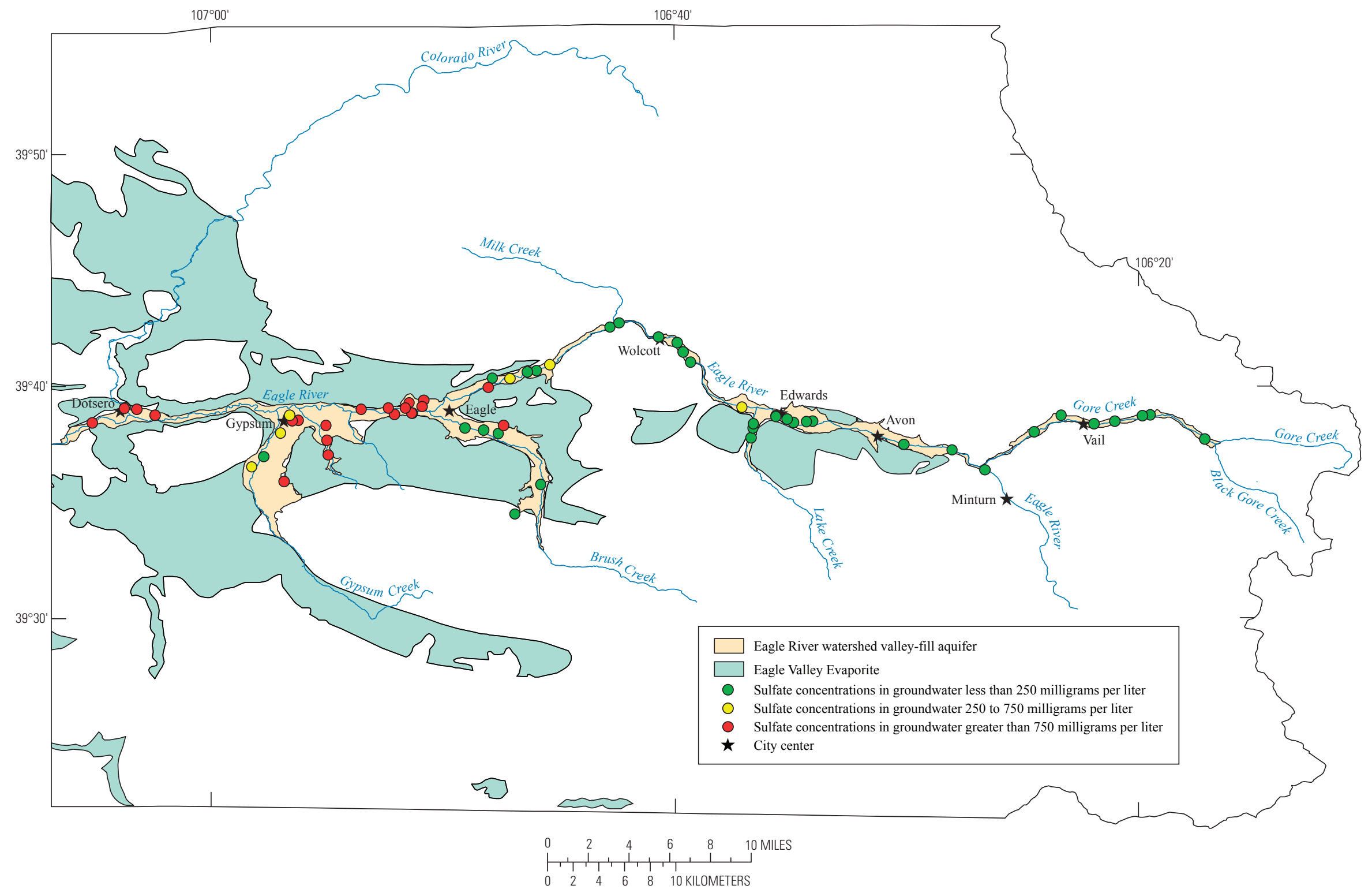

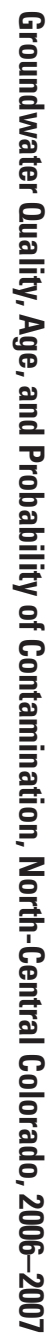

Figure 7. Sulfate concentrations in groundwater in the Eagle River watershed valley-fill aquifer, 2006-2007, Eagle County, Colorado. 
Table 8. Summary statistics for major ions, nutrients, stable isotopes of hydrogen and oxygen, and tritium in groundwater, Eagle River watershed, 2006-2007, Eagle County, Colorado.

$\left[\mathrm{CaCO}_{3}\right.$, calcium carbonate; ${ }^{\circ} \mathrm{C}$, degrees Celsius; diss., dissolved; lab., laboratory analysis, $\mu \mathrm{S} / \mathrm{cm}$, microsiemens per centimeter; mg/L, milligrams per liter; $\mu \mathrm{g} / \mathrm{L}$, micrograms per liter; $\mathrm{N}$, nitrogen; P, phosphorus; pCi/L, picocuries per liter; RD, relative difference; --, no data; \%, per mil; na, not applicable; <, less than; bold letters indicate concentrations above National Secondary Drinking Water Regulations (U.S. Environmental Protection Agency, 2003)]

\begin{tabular}{|c|c|c|c|c|c|c|c|c|c|c|}
\hline \multirow[b]{2}{*}{ Constituent } & \multirow{2}{*}{$\begin{array}{c}\text { No. of } \\
\text { wells } \\
\text { sampled }\end{array}$} & \multirow{2}{*}{$\begin{array}{l}\text { Laboratory } \\
\text { reporting } \\
\text { level }\end{array}$} & \multirow{2}{*}{$\begin{array}{c}\text { Minimum } \\
\text { concentration } \\
\text { measured }\end{array}$} & \multicolumn{5}{|c|}{ Percentile } & \multirow{2}{*}{$\begin{array}{l}\text { Maximum } \\
\text { concentration } \\
\text { measured }\end{array}$} & \multirow{2}{*}{$\begin{array}{c}\text { National } \\
\text { Secondary } \\
\text { Drinking Water } \\
\text { Regulation }\end{array}$} \\
\hline & & & & 10 & 25 & 50 & 75 & 90 & & \\
\hline Specific conductance, $\mu \mathrm{S} / \mathrm{cm}$ at $25^{\circ} \mathrm{C}$ & 61 & $<3$ & 239 & 420 & 601 & 917 & 2,140 & 2,967 & 8,100 & \\
\hline $\mathrm{pH}$, standard units & 61 & $<0.1$ & 7.0 & 7.1 & 7.2 & 7.4 & 7.6 & 7.7 & 8.6 & $6.5-8.5$ \\
\hline Temperature, degrees Celsius & 61 & na & 3.9 & 8.0 & 9.4 & 10.4 & 11.4 & 12.6 & 16.2 & \\
\hline Dissolved oxygen, $\mathrm{mg} / \mathrm{L}$ & 61 & $<.1$ & $<0.1$ & 0.4 & 2.2 & 4.0 & 5.6 & 6.4 & 8.6 & \\
\hline Hydrogen sulfide, in $\mathrm{mg} / \mathrm{L}$ & 61 & $<.1$ & $<.1$ & $<0.1$ & $<0.1$ & $<0.1$ & $<0.1$ & $<0.1$ & 0.18 & \\
\hline Calcium, diss., mg/L & 61 & $<.02$ & 3.8 & 52 & 79 & 112 & 432 & 538 & 621 & \\
\hline Magnesium, diss., mg/L & 61 & $<.014$ & 0.717 & 7.77 & 17 & 23 & 56 & 107 & 321 & \\
\hline Sodium, diss., $\mathrm{mg} / \mathrm{L}$ & 61 & $<.20$ & 3.8 & 5.3 & 7.4 & 27 & 58 & 184 & 1,220 & \\
\hline Potassium, diss., $\mathrm{mg} / \mathrm{L}$ & 61 & $<.04$ & 0.53 & 1.2 & 1.5 & 2.6 & 3.7 & 11 & 34 & \\
\hline Bicarbonate, diss, field, $\mathrm{mg} / \mathrm{L}$ as $\mathrm{HCO}_{3}$ & 51 & $<5$ & 77 & 164 & 214 & 245 & 299 & 344 & 513 & \\
\hline Alkalinity, diss., field, $\mathrm{mg} / \mathrm{L}$ as $\mathrm{CaCO}_{3}{ }^{3}$ & 60 & $<5$ & 63 & 134 & 176 & 199 & 241 & 277 & 420 & \\
\hline Sulfate, diss., $\mathrm{mg} / \mathrm{L}$ & 61 & $<.18$ & 4.3 & 15 & 80 & 170 & 1,065 & 1,430 & 2,400 & 250 \\
\hline Chloride, diss., $\mathrm{mg} / \mathrm{L}$ & 61 & $<.12$ & 1.1 & 3.1 & 13 & 34 & 72 & 233 & 2,000 & 250 \\
\hline Fluoride, diss., mg/L & 61 & $<.10$ & $<.10$ & $<.10$ & 0.12 & 0.17 & 0.24 & 0.33 & 5.4 & 2.0 \\
\hline Silica, diss., $\mathrm{mg} / \mathrm{L}$ & 61 & $<.20$ & 5.4 & 7.2 & 9.4 & 13 & 14 & 17 & 25 & \\
\hline Total dissolved solids, $\mathrm{mg} / \mathrm{L}$ & 61 & na & 137 & 225 & 350 & 587 & 1,770 & 2,508 & 5,320 & 500 \\
\hline Ammonia, diss., mg/L as $\mathrm{N}$ & 61 & $<.02$ & $<.02$ & $<.02$ & 0.02 & 0.02 & 0.02 & 0.03 & 2.0 & \\
\hline Nitrite, diss., $\mathrm{mg} / \mathrm{L}$ as $\mathrm{N}$ & 61 & $<.002$ & $<.002$ & $<.002$ & $<.002$ & $<.002$ & $<.002$ & $<.002$ & 0.007 & \\
\hline Nitrite plus nitrate, diss., $\mathrm{mg} / \mathrm{L}$ as $\mathrm{N}$ & 61 & $<.06$ & $<.06$ & $<.06$ & 0.36 & 0.74 & 1.3 & 2.5 & 5.4 & \\
\hline Orthophosphate, diss., $\mathrm{mg} / \mathrm{L}$ as $\mathrm{P}$ & 61 & $<.006$ & $<.006$ & $<.006$ & $<.006$ & $<.006$ & 0.008 & 0.018 & 0.110 & \\
\hline Boron, diss., $\mu \mathrm{g} / \mathrm{L}$ & 61 & $<1.8$ & 5.4 & 11 & 21 & 37 & 90 & 173 & 332 & \\
\hline Iron, diss., $\mu \mathrm{g} / \mathrm{L}$ & 61 & $<6$ & 3 & 6 & 6 & 16 & 61 & 336 & 8,360 & 300 \\
\hline Manganese, diss., $\mu \mathrm{g} / \mathrm{L}$ & 61 & $<.2$ & 0.1 & 0.2 & 0.4 & 1.7 & 12 & 105 & 1,500 & 50 \\
\hline Hydrogen $2 / 1$ ratio, per mil & 61 & $<2$ & -140 & -130.4 & -128 & -126 & -125 & -122.2 & -117 & \\
\hline Oxygen $18 / 16$ ratio, per mil & 61 & $<0.20$ & -18.05 & -17.43 & -17.24 & -17.03 & -16.89 & -16.38 & -16.14 & \\
\hline Tritium, $\mathrm{pCi} / \mathrm{L}$ & 61 & $<1.00$ & $<1.00$ & 20.11 & 24.88 & 28.16 & 31.44 & 34.5 & 45.8 & \\
\hline
\end{tabular}




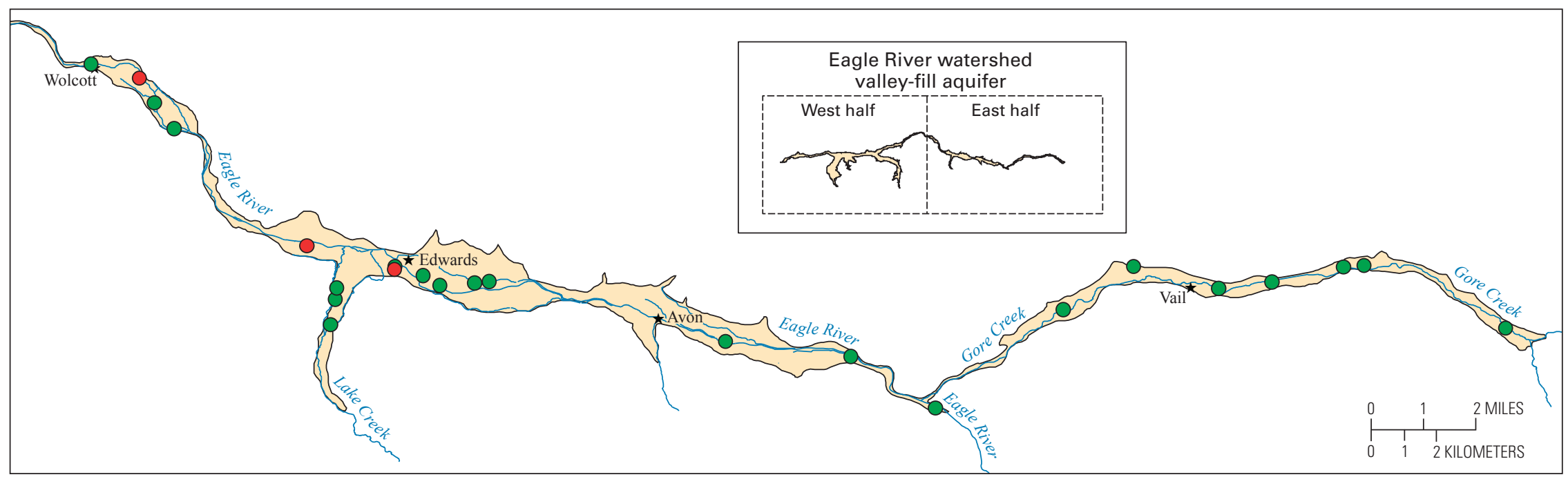

WEST HALF OFTHE EAGLE RIVER WATERSHED VALLEY-FILL AOUIFER

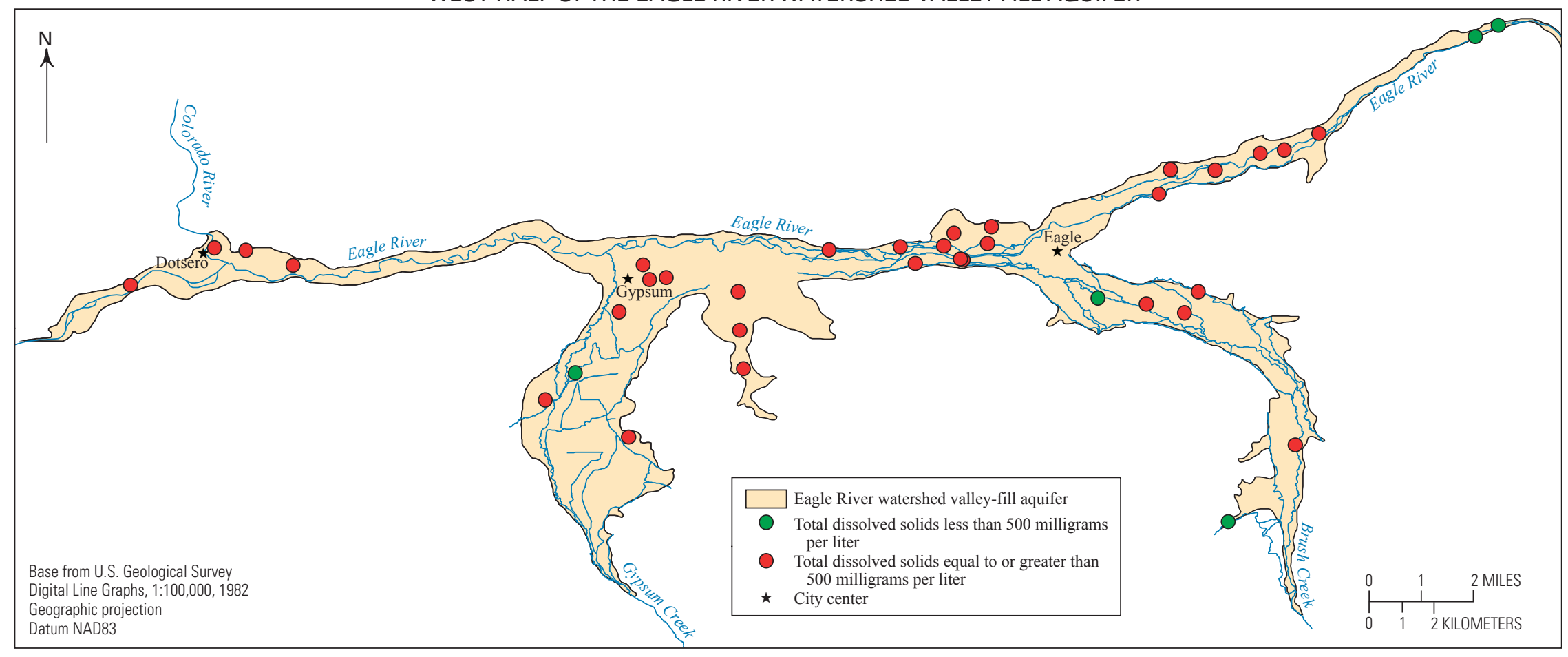

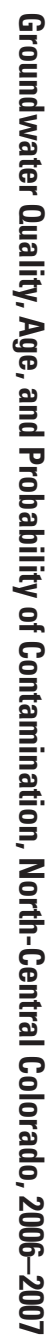

Figure 8. Total dissolved-solids concentrations in groundwater in the Eagle River watershed valley-fill aquifer, 2006-2007, Eagle County, Colorado. 
interbeds of shale, limestone, and sandstone (Day and others, 1999). Sediments eroded from the Eagle Valley Evaporite were incorporated into the aquifer materials of the ERWVFA, causing high sulfate and total dissolved solids concentrations in groundwater that flows through the aquifer.

\section{Nutrients}

Nitrate concentrations in groundwater were generally low; the median nitrate concentration was about $0.74 \mathrm{mg} / \mathrm{L}$, and the maximum concentration measured $5.4 \mathrm{mg} / \mathrm{L}$ (table 2, table 8). Concentrations of ammonia and nitrite as nitrogen in groundwater of the ERWVFA were close to, or below, the laboratory reporting level in most samples. Most groundwater samples contained dissolved oxygen concentrations greater than $0.5 \mathrm{mg} / \mathrm{L}$, dissolved manganese concentrations less than $50 \mu \mathrm{g} / \mathrm{L}$, and dissolved iron concentrations less than $100 \mu \mathrm{g} / \mathrm{L}$, indicating they have oxidized geochemical conditions (Paschke and others, 2007, p. 1-12). Under oxidized conditions, nitrate can exist in groundwater for many years. For instance, the High Plains aquifer has mostly oxidized geochemical conditions; McMahon and others (2007) estimate that it could take between 250 and 14,000 years for nitrate concentrations in the High Plains aquifer to decrease by $1 \mathrm{mg} / \mathrm{L}$. Although there are no estimates of actual denitrification rates in the ERWVFA, the oxidized conditions observed in most areas of the ERWVFA indicate that nitrate from fertilizers and animal or human waste could persist for several decades in groundwater of the ERWVFA.

Nitrate concentrations of natural recharge water to the ERWVFA were determined by examining precipitation data collected by the National Atmospheric Deposition Program (2007). Precipitation is a good indicator of nitrate concentrations in natural recharge water to the ERWVFA because it establishes nitrate concentrations before any anthropogenic or hydrogeologic effects. There were no National Atmospheric Deposition Program (NADP) collection sites located in Eagle County, so data from three NADP sites that surround Eagle County were evaluated: Niwot Saddle, Sand Spring, and Sunlight Peak (fig. 9). Annual data on precipitation-weighted mean concentrations of ammonia and nitrate were downloaded (http://nadp.sws.uiuc.edu/sites/sitemap.asp? state=co) for calendar years 1988 through 2006, which was the period of record in common at the three sites. The median ammonia concentration at all three NADP sites combined was $0.10 \mathrm{mg} / \mathrm{L}$, and the median nitrate concentration at all three sites combined was $0.20 \mathrm{mg} / \mathrm{L}$ (table 9). There were minimal differences in ammonia, nitrate, and total nitrogen concentrations among the three sites, indicating that concentrations in precipitation were relatively constant near Eagle County (table 9). Total inorganic nitrogen concentrations were calculated by adding the ammonia and nitrate concentrations; median total nitrogen concentrations of precipitation at all three sites combined was $0.30 \mathrm{mg} / \mathrm{L}$, the maximum concentration was 0.63 , and the standard deviation was 0.1 . The largest

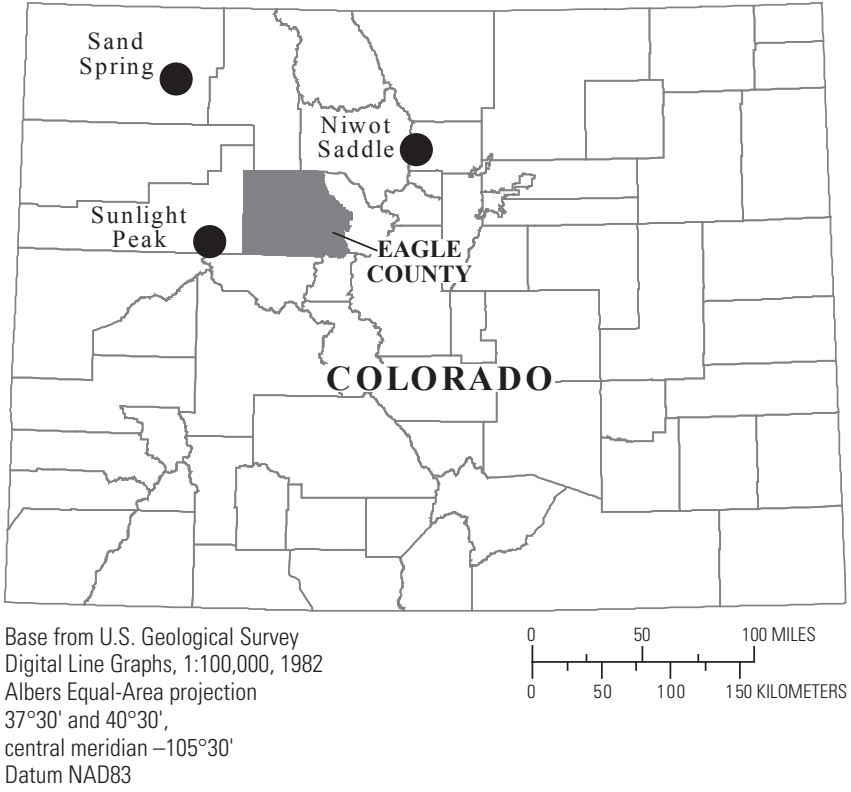

Figure 9. Locations of the National Atmospheric Deposition Program precipitation sites, north-central Colorado.

Table 9. Summary statistics for ammonia and nitrate concentrations in precipitation measured by the National Atmospheric Deposition Program (2007) at the Niwot Saddle, Sand Spring, and Sunlight Peak sites, Colorado, 1988-2006.

$[\mathrm{N}$, nitrogen; $\mathrm{mg} / \mathrm{L}$, milligrams per liter $]$

\begin{tabular}{|c|c|c|c|}
\hline Statistic & $\begin{array}{c}\text { Ammonia } \\
\text { as N }\end{array}$ & $\begin{array}{c}\text { Nitrate } \\
\text { as N }\end{array}$ & $\begin{array}{c}\text { Total } \\
\mathbf{N}\end{array}$ \\
\hline \multicolumn{4}{|c|}{ Niwot Saddle, Sand Spring, and Sunlight Peak combined } \\
\hline Minimum concentration $(\mathrm{mg} / \mathrm{L})$ & 0.04 & 0.12 & 0.17 \\
\hline Mean concentration $(\mathrm{mg} / \mathrm{L})$ & 0.12 & 0.21 & 0.32 \\
\hline Median concentration $(\mathrm{mg} / \mathrm{L})$ & 0.10 & 0.20 & 0.30 \\
\hline Maximum concentration $(\mathrm{mg} / \mathrm{L})$ & 0.29 & 0.35 & 0.63 \\
\hline Standard deviation $(\mathrm{mg} / \mathrm{L})$ & 0.05 & 0.05 & 0.10 \\
\hline \multicolumn{4}{|c|}{ Niwot Saddle } \\
\hline Minimum concentration (mg/L) & 0.04 & 0.13 & 0.17 \\
\hline Mean concentration $(\mathrm{mg} / \mathrm{L})$ & 0.11 & 0.21 & 0.33 \\
\hline Median concentration $(\mathrm{mg} / \mathrm{L})$ & 0.10 & 0.20 & 0.30 \\
\hline Maximum concentration $(\mathrm{mg} / \mathrm{L})$ & 0.19 & 0.30 & 0.50 \\
\hline Standard deviation $(\mathrm{mg} / \mathrm{L})$ & 0.04 & 0.04 & 0.08 \\
\hline \multicolumn{4}{|c|}{ Sand Spring } \\
\hline Minimum concentration (mg/L) & 0.06 & 0.18 & 0.24 \\
\hline Mean concentration $(\mathrm{mg} / \mathrm{L})$ & 0.15 & 0.25 & 0.40 \\
\hline Median concentration $(\mathrm{mg} / \mathrm{L})$ & 0.14 & 0.24 & 0.38 \\
\hline Maximum concentration $(\mathrm{mg} / \mathrm{L})$ & 0.29 & 0.35 & 0.63 \\
\hline Standard deviation $(\mathrm{mg} / \mathrm{L})$ & 0.06 & 0.05 & 0.11 \\
\hline \multicolumn{4}{|c|}{ Sunlight Peak } \\
\hline Minimum concentration $(\mathrm{mg} / \mathrm{L})$ & 0.05 & 0.12 & 0.17 \\
\hline Mean concentration $(\mathrm{mg} / \mathrm{L})$ & 0.09 & 0.15 & 0.24 \\
\hline Median concentration (mg/L) & 0.08 & 0.15 & 0.24 \\
\hline Maximum concentration $(\mathrm{mg} / \mathrm{L})$ & 0.18 & 0.19 & 0.37 \\
\hline Standard deviation $(\mathrm{mg} / \mathrm{L})$ & 0.03 & 0.02 & 0.05 \\
\hline
\end{tabular}


expected total nitrogen concentration of $0.74 \mathrm{mg} / \mathrm{L}$ was calculated by adding the maximum concentration measured at all three sites plus the largest standard deviation. This indicated that concentrations of nitrate in natural recharge water to the ERWVFA were less than $1 \mathrm{mg} / \mathrm{L}$. In a national study, Nolan and Hitt (2002) also reported that concentrations of nitrate in groundwater in undeveloped forested areas of the United States were less than $1 \mathrm{mg} / \mathrm{L}$.

More than 50 percent of the nitrate concentrations in the ERWVFA were less than $1 \mathrm{mg} / \mathrm{L}$ (table 8), indicating that water from more than 50 percent of the wells tested in the ERWVFA had nitrate concentrations similar to natural recharge water (precipitation). Nitrate concentrations were larger than concentrations of precipitation near Edwards, Eagle, Gypsum, and Dotsero (fig. 10). There were four major sources of nitrogen in the Eagle River watershed that could cause nitrate concentrations in excess of that in precipitation: fertilizers, manure, natural sources, and sewer systems. Agricultural uses of fertilizer were very limited in the Eagle River watershed, so probably the largest use of fertilizers was on turf grass, although rangeland areas are sometimes fertilized to improve winter forage for deer and elk. Manure from cattle, dogs, and horses can provide excess nitrogen to groundwater. As the result of evapotranspiration, nitrate can naturally accumulate in soils in arid environments (such as the environment near Gypsum and Dotsero), which leaves chloride and nitrate salts that can leach to groundwater following changes in land use or climate (Walvoord and others, 2003). Excess nitrogen input to groundwater can result from conventional domestic septic systems, from leaking municipal sewer systems, and from discharge of treated effluent to surface water and the land surface.

In an attempt to determine the source of elevated nitrate concentrations, boron was analyzed in groundwater samples from the ERWVFA. Boron can be an indicator of wastewater effluent from septic and sewer systems because boron is used as a bleaching additive in soaps and detergents (Westgate and others, 2000; Barber and others, 1988; Thurman and others, 1986; Verstraeten and others, 2005; Vengosh and others, 1994; Leenheer and others, 2001). Boron is a conservative tracer because it is biochemically inactive in groundwater (Barber and others, 1988; Ford and Tellam, 1994; Leenhouts and others, 1998). Boron can also be naturally concentrated in soils in arid climates, such as the climate near Dotsero, and be associated with evaporite deposits such as gypsum. Boron does not appear to be a useful indicator of nitrate from wastewater in the ERWVFA (fig. 11). There was a relatively strong relation between boron and sulfate concentrations, indicating that boron in groundwater from the ERWVFA may be associated with the same gypsum deposits that are the source of the elevated sulfate (fig. 11). To quantify this correlation, boron and sulfate were correlated using Spearman's rho. Two factors are strongly correlated when Spearman's rho approaches plus or minus one; no correlation exists as the Spearman's rho approaches zero (Helsel and Hirsch, 1992). There is a strong correlation between boron and sulfate (Spearman's rho $=0.9$ ). Nitrate concentrations are shown as colored dots in figure 11; nitrate concentrations greater than $1 \mathrm{mg} / \mathrm{L}$ are detected in a wide range of boron and sulfate concentrations, indicating there is not a strong relation between boron and nitrate (Spearman's rho $=0.2$ ). If a strong relation existed between boron and nitrate, most nitrate detections greater than $1 \mathrm{mg} / \mathrm{L}$ would be associated with high boron concentrations. Elevated chloride concentrations in groundwater can also be an indicator of wastewater effluent. Groundwater with significant amounts of wastewater effluent should have elevated concentrations of boron, chloride, and nitrate, but there is no clear relation between boron, chloride, and nitrate in the right-hand graph in figure 11 (Spearman's rho $=0.2$ ). A few individual wells may have had elevated nitrate concentrations from septic or sewage effluent, but boron was not a useful indicator of that effluent. Isotopes of boron $\left({ }^{11} \mathrm{~B} /{ }^{10} \mathrm{~B}\right)$ can be used to differentiate between naturally occurring boron and that from sewage (Naftz and others, 2008). Forcada and Evangelista (2008) indicate that boron isotopes may be useful for identifying wastewater in high-sulfate water, so sampling for boron isotopes may be useful in future studies of the ERWVFA. Examining chloride/ bromide ratios of groundwater may also be a useful indicator of effluent (Vengosh and Pankratov, 1998).

\section{Tritium}

Tritium, when plotted with one of the other CFC compounds, can be an effective tool for groundwater age dating and for determining amounts of groundwater mixing (Plummer and others, 2003). In the Eagle River watershed, tritium and $\mathrm{CFC}-11$ were useful indicators of groundwater age (fig. 12). The solid blue line in figure 12 is the intersection of tritium activities in precipitation decayed to the average year of sampling (2007) with the northern hemisphere CFC-11 atmospheric mixing ratio over time and is commonly called the piston-flow line because groundwater recharged from precipitation and moving in the aquifer under unmixed piston flow will plot on that line. Points that plot near the line are composed of waters that were derived from precipitation falling at that time in history and that was recharged to groundwater. The year of recharge is shown on the line. It is common for groundwater sampled from wells to be mixtures of young and old waters that were mixed in the well bore, particularly in wells with screen lengths greater than $1.5 \mathrm{~m}$. Points plotting below the piston-flow line either contain a significant fraction of old (prenuclear detonation) water or are located in areas of deep unsaturated zones and(or) low recharge rate, which moves the points toward the origin of the graph (fig. 12). Samples plotting above the piston-flow line contain fractions of water elevated in tritium from the mid-1960s period of atmospheric testing of nuclear weapons. In the Eagle River watershed, a useful indicator of the predisposition of water to anthropogenic contamination is whether the water is composed of mostly young waters (high predisposition) or contain a significant portion of older waters (lower predisposition). As will be shown later in the "Comparison of Probability Models" section of this report, an effective model was developed using logistic regression to predict the probability of detecting unmixed young groundwater in the ERWVFA by using $\mathrm{CFC}-11$ concentrations and tritium activities in groundwater. 


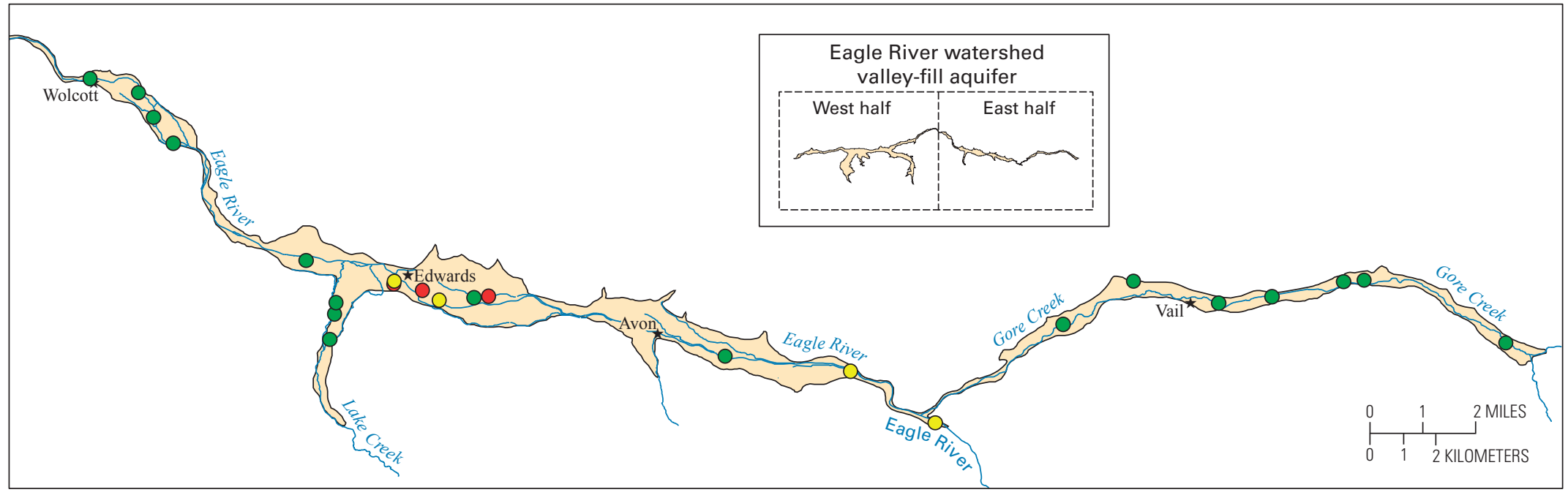

WEST HALF OFTHE EAGLE RIVER WATERSHED VALLEY-FILL AQUIFER

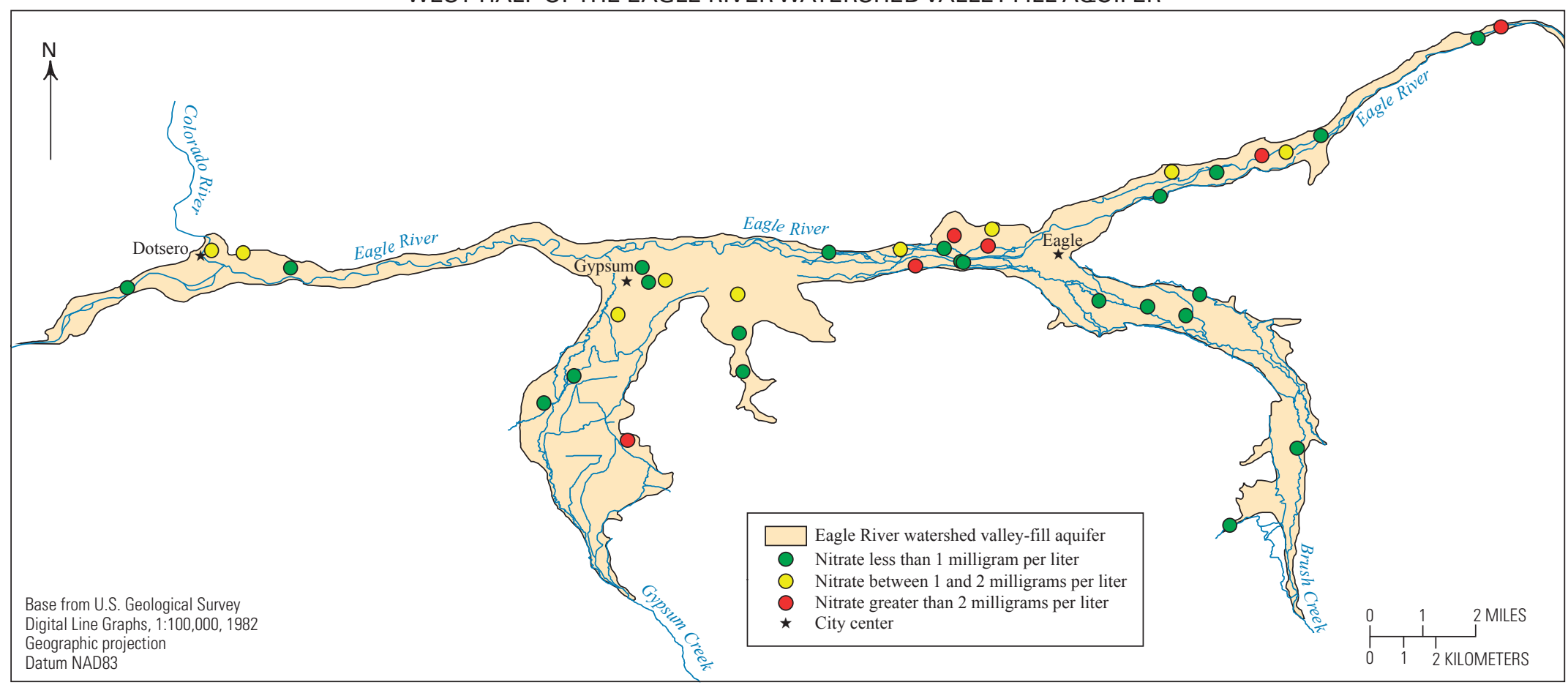

Figure 10. Nitrate concentrations in groundwater in the Eagle River watershed valley-fill aquifer, 2006-2007, Eagle County, Colorado. 

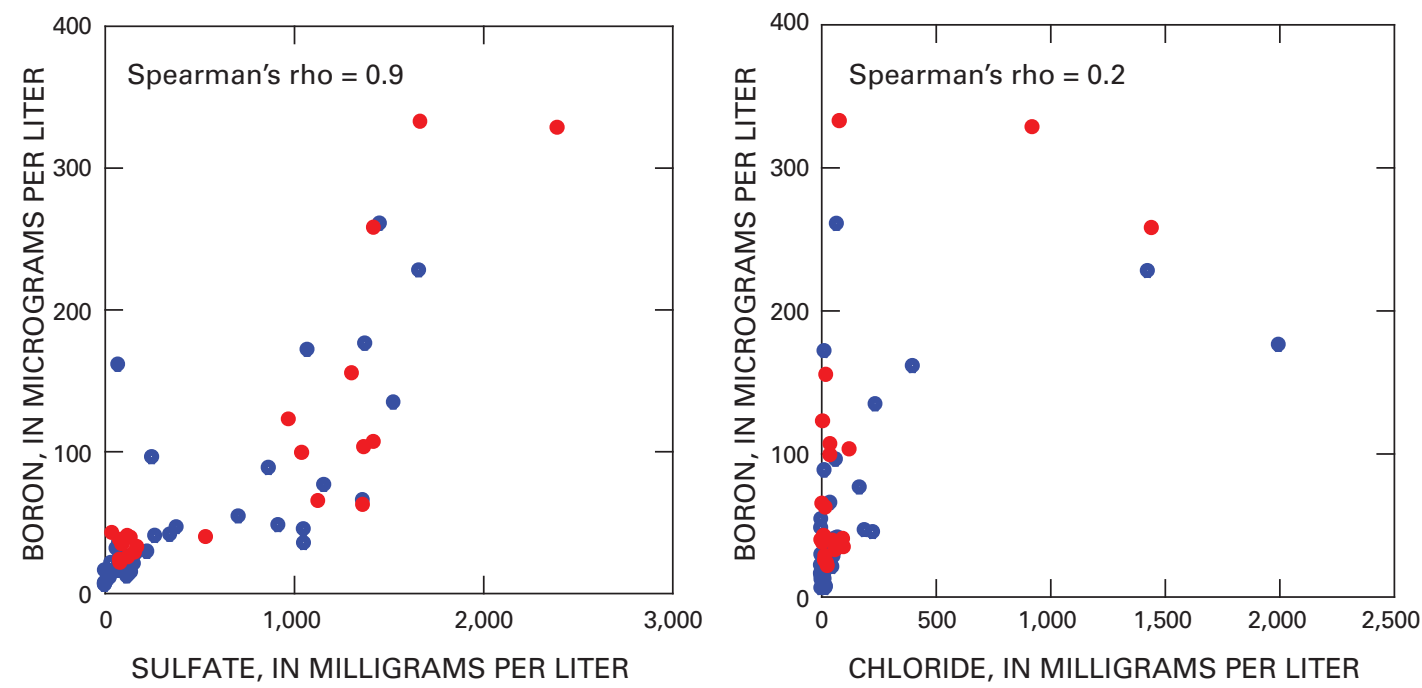

EXPLANATION

- Nitrate greater than 1 milligram per liter

- Nitrate equal to or less than

1 milligram per liter

Figure 11. Boron, chloride, sulfate, and nitrate concentrations in groundwater, Eagle River watershed valley-fill aquifer, 2006-2007, Eagle County, Colorado.

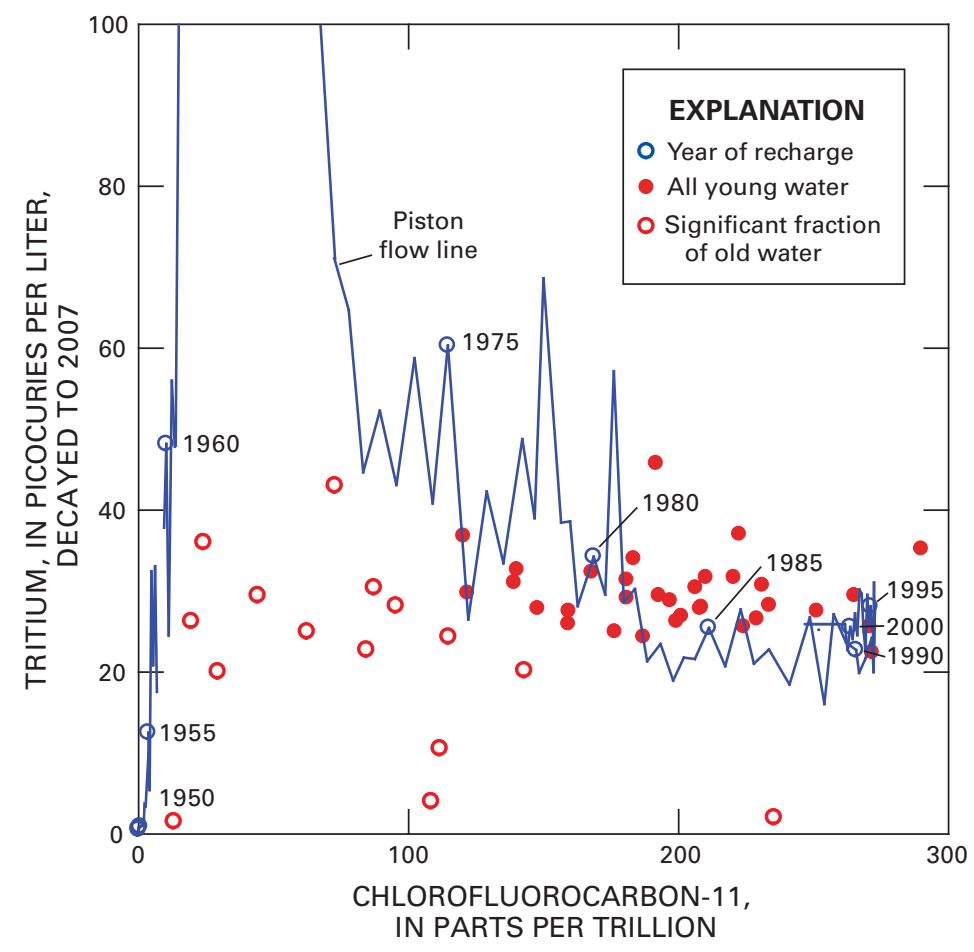

Figure 12. Measured CFC-11 concentrations and tritium activities in groundwater, Eagle River watershed valley-fill aquifer, 2006-2007, Eagle County, Colorado. 


\section{Dissolved Gases}

Dissolved gas concentrations, as well as the ratios of concentrations, can be used to calculate the recharge temperature of a groundwater sample (table 10) because the solubility of gases in water varies as a function of temperature (Plummer and others, 2004). Determining the groundwater temperature is important because groundwater ages determined using CFCs are based on Henry's law solubility, which is dependent on the groundwater temperature at time of recharge and altitude at location of recharge. About one-half of the groundwater sites were sampled for dissolved gases, but the areal distribution of the sites was appropriate to show a range of recharge dates throughout the ERWVFA. Recharge temperatures for groundwater sites without dissolved-gas data were extrapolated from adjacent groundwater sites with dissolved-gas data. The field temperature measured at the time of sampling was used for recharge temperatures at surface-water sites. Land-surface elevations were used for recharge elevations at all sites because actual recharge locations were unknown for most sites and groundwater ages are less sensitive to recharge elevations than recharge temperatures (Plummer and Busenberg, 1999).

Dissolved-gas samples were collected in replicate from each site; samples were analyzed for $\mathrm{Ar}, \mathrm{CO}_{2}, \mathrm{CH}_{4}, \mathrm{O}_{2}$, and $\mathrm{N}_{2}$ (table 10). Most samples contained excess air, probably from excess air being trapped and dissolved in groundwater under increased hydrostatic pressure during rapid recharge conditions. Mean recharge temperatures at each site ranged from 1.1 to $64.0^{\circ} \mathrm{C}\left(34\right.$ to $\left.147^{\circ} \mathrm{F}\right)$. Median recharge temperature of all sites combined was $7.3^{\circ} \mathrm{C}\left(45^{\circ} \mathrm{F}\right)$ (table 10$)$. Mean and median field water temperatures measured at the time of sampling were about $3.1^{\circ} \mathrm{C}\left(5.6^{\circ} \mathrm{F}\right)$ higher than the temperatures determined using dissolved gases (table 10), indicating that groundwaters at the time of sampling probably recharged at lower temperatures than current groundwater temperatures. Site 27 (table 10) had an anomalously high recharge temperature of $64^{\circ} \mathrm{C}\left(147^{\circ} \mathrm{F}\right)$. Water from this well contained an anomalously high concentration of methane and anomalously low concentrations of the other gases. Apparently this sample has undergone a more complex evolution than any of the other samples from the study area, and recharge temperature cannot be determined from the nitrogen and argon concentrations in this sample. If dissolved-gas data from this well are eliminated from the analysis, then the minimum, maximum, and median recharge temperatures of the groundwater samples are $1.1,13.2$ and $7.2^{\circ} \mathrm{C}\left(34,56\right.$, and $\left.45^{\circ} \mathrm{F}\right)$, respectively.

Long-term mean annual daily air temperature data collected by the Colorado Climate Center (http://ccc.atmos.colostate.edu/ dly form. html) were comparable to recharge temperatures determined using dissolved gases. Two sites in the Eagle River watershed have long-term temperature data: one site at the Eagle County Airport (1949 through 1993) and one site at Vail (1986 through 1999). Mean annual daily air temperature at the Eagle County Airport for calendar years 1986 through 1993, which was the period of record in common at both sites, was $6.4^{\circ} \mathrm{C}$ $\left(43.5^{\circ} \mathrm{F}\right)$; the median annual air temperature was $7.5^{\circ} \mathrm{C}\left(45.5^{\circ} \mathrm{F}\right)$.
Mean annual daily air temperature at Vail for calendar years 1986 through 1993 was $0.2^{\circ} \mathrm{C}\left(32.4^{\circ} \mathrm{F}\right)$; the median annual air temperature was $1.7^{\circ} \mathrm{C}\left(35.0^{\circ} \mathrm{F}\right)$. The differences in temperatures between Vail and the Eagle County Airport are because the Eagle County Airport is at a much lower elevation $(1,980 \mathrm{~m}[6,500 \mathrm{ft}])$ than Vail $(2,510 \mathrm{~m}[8,230 \mathrm{ft}])$.

\section{Chlorofluorocarbons and Groundwater Age}

Groundwater ages of water in the Eagle River watershed were estimated using CFCs, excess air, recharge temperature, and recharge elevation (table 3 ). Five replicate CFC samples were collected at each site, and in most cases three of those samples were analyzed for each site and the concentrations were averaged. In cases where there was high variability in CFC concentrations between each replicate sample, additional samples were analyzed for each site up to the maximum of five samples. Groundwater ages were based on CFC atmospheric mixing ratios, which were calculated from the $\mathrm{CFC}$ concentrations using Henry's law (Busenberg and Plummer, 1992; Plummer and Busenberg, 1999; International Atomic Energy Agency, 2006) and estimates of excess air, recharge temperature, and recharge elevation (table 3). Most groundwater ages and estimates of percentage of young water (percentage of water younger than 1940) were determined using either CFC-113/CFC-11 ratios or piston-flow apparent ages based on $\mathrm{CFC}-113$ (International Atomic Energy Agency, 2006).

The median groundwater recharge date of all sites combined is 1989 and the standard deviation was about 9 years, indicating that most groundwater in the ERWVFA was young water (table 3 , fig. 13). The median percentage of young water was 83 percent and the standard deviation was about 28 percent, indicating that only a portion of water from most wells was composed of old (older than 1940) water. To put the groundwater age data in the context of the probability of groundwater contamination, most groundwaters in the ERWVFA were recently recharged waters and have a high predisposition to anthropogenic contamination.

Two sites (11 and 47, table 3) were sampled during 2006 and 2007, which allowed a check on the comparability of the groundwater recharge dates between the two sampling events. The two sites were sampled about one year apart, and groundwater recharge dates were about one year different between the two sampling events, indicating there is good repeatability in the groundwater age determinations. These sites were originally selected for resampling to verify the relatively large tetrachloroethene concentrations detected at site 11 (table 4) and the relatively large nitrate concentration detected at site 47 (table 2). The resampling also confirmed the tetrachloroethene and nitrate concentrations.

CFC -12 concentrations exceeded those in water in equilibrium with the modern atmosphere in many samples, so in most cases groundwater ages could not be determined using $\mathrm{CFC}-12$. The maximum expected concentration of 
Table 10. Dissolved-gas data analyzed from groundwater samples collected from the Eagle River watershed valley-fill aquifer, 2006-2007, Eagle County, Colorado.

$\left[{ }^{\circ} \mathrm{C}\right.$, degrees Celsius; m, month; d, day; y, year; mg/L, milligrams per liter; NAVD 29, National Geodetic Vertical Datum of 1929; trace, detected, but at very low concentration; Mean concentrations were determined by averaging replicate samples collected at each site]

\begin{tabular}{|c|c|c|c|c|c|c|c|c|c|c|c|c|}
\hline $\begin{array}{c}\text { Local } \\
\text { identification } \\
\text { number } \\
\text { (see table } 1 \\
\text { and fig. 2) }\end{array}$ & $\begin{array}{c}\text { U.S. Geological } \\
\text { Survey station } \\
\text { identification } \\
\text { number }\end{array}$ & $\begin{array}{l}\text { Date of } \\
\text { sample } \\
\text { collection } \\
\text { (mmddyy) }\end{array}$ & $\begin{array}{l}\text { Field water } \\
\text { temperature } \\
\text { measured at } \\
\text { the time of } \\
\text { sample } \\
\text { collection } \\
\left({ }^{\circ} \mathrm{C}\right)\end{array}$ & $\begin{array}{c}\text { Recharge } \\
\text { elevation } \\
\text { (feet above } \\
\text { NGVD 29) }\end{array}$ & $\begin{array}{c}\text { Mean } \\
\text { argon } \\
(\mathrm{Ar}) \\
(\mathrm{mg} / \mathrm{L})\end{array}$ & $\begin{array}{c}\text { Mean } \\
\text { carbon } \\
\text { dioxide } \\
\left(\mathrm{CO}_{2}\right) \\
(\mathrm{mg} / \mathrm{L})\end{array}$ & $\begin{array}{c}\text { Mean } \\
\text { methane } \\
\left(\mathrm{CH}_{4}\right) \\
(\mathrm{mg} / \mathrm{L})\end{array}$ & $\begin{array}{c}\text { Mean } \\
\text { oxygen } \\
\left(\mathrm{O}_{2}\right) \\
(\mathrm{mg} / \mathrm{L})\end{array}$ & $\begin{array}{c}\text { Mean } \\
\text { nitrogen } \\
\left(\mathrm{N}_{2}\right) \\
(\mathrm{mg} / \mathrm{L})\end{array}$ & $\begin{array}{c}\text { Mean } \\
\text { excess } \\
\text { nitrogen } \\
\left(\mathrm{N}_{2}\right) \\
(\mathrm{mg} / \mathrm{L})\end{array}$ & $\begin{array}{c}\text { Mean } \\
\text { recharge } \\
\text { temperature } \\
\left({ }^{\circ} \mathrm{C}\right)\end{array}$ & $\begin{array}{l}\text { Mean excess air } \\
\text { in water sample } \\
\text { (cubic centimeters } \\
\text { per liter at standard } \\
\text { temperature } \\
\text { and pressure) }\end{array}$ \\
\hline 9 & 393823106215900 & $06 / 06 / 07$ & 3.8 & 8,189 & 0.7 & 8.5 & 0.0 & 5.4 & 18.0 & 0.0 & 1.1 & 1.4 \\
\hline 10 & 393844106232300 & $06 / 06 / 07$ & 9.0 & 8,180 & 0.6 & 39.7 & 0.0 & 4.2 & 17.7 & 0.0 & 3.4 & 2.1 \\
\hline 11 & 393802106243501 & $08 / 24 / 06$ & 9.0 & 7,970 & 0.6 & 17.5 & 0.0 & 0.6 & 17.7 & 0.0 & 8.3 & 3.7 \\
\hline 12 & 393623106264201 & $06 / 11 / 07$ & 8.0 & 7,755 & 0.6 & 9.2 & 0.0 & 3.4 & 16.9 & 0.0 & 5.7 & 2.0 \\
\hline 13 & 393715106280701 & $06 / 19 / 07$ & 9.5 & 7,611 & 0.6 & 16.2 & 0.0 & 3.8 & 17.7 & 0.0 & 4.1 & 2.0 \\
\hline 15 & 393830106340601 & $06 / 11 / 07$ & 9.8 & 7,380 & 0.6 & 13.1 & 0.0 & 7.1 & 17.4 & 0.0 & 5.8 & 2.2 \\
\hline 17 & 393826106345601 & $08 / 24 / 06$ & 9.4 & 7,267 & 0.6 & 17.1 & 0.0 & 3.7 & 16.5 & 0.0 & 8.1 & 2.1 \\
\hline 18 & 393836106351201 & $06 / 08 / 07$ & 9.4 & 7,248 & 0.6 & 18.0 & 0.0 & 4.5 & 16.1 & 0.0 & 7.4 & 1.4 \\
\hline 19 & 393844106354001 & $05 / 21 / 07$ & 9.4 & 7,213 & 0.6 & 11.4 & 0.0 & 2.9 & 16.4 & 0.0 & 6.1 & 1.2 \\
\hline 21 & 393748106364201 & $06 / 08 / 07$ & 9.2 & 7,387 & 0.6 & 8.5 & 0.0 & 5.6 & 16.3 & 0.0 & 7.2 & 1.6 \\
\hline 27 & 394153106395501 & $06 / 09 / 07$ & 15.4 & 6,973 & 0.2 & 1.3 & 19.7 & 0.0 & 4.1 & 0.0 & 64.0 & -0.6 \\
\hline 32 & 394040106455701 & $05 / 15 / 07$ & 11.2 & 6,685 & 0.6 & 24.7 & 0.0 & 2.6 & 15.9 & 0.0 & 8.3 & 1.3 \\
\hline 35 & 394020106475101 & $08 / 25 / 06$ & 13.0 & 6,704 & 0.7 & 39.1 & 0.0 & 4.8 & 21.1 & 0.0 & 3.8 & 4.8 \\
\hline 36 & 393956106480201 & $05 / 16 / 07$ & 10.5 & 6,660 & 0.6 & 61.9 & 0.0 & 0.4 & 17.2 & 0.0 & 8.2 & 2.5 \\
\hline 37 & 393431106465301 & $06 / 12 / 07$ & 7.5 & 7,618 & 0.6 & 29.8 & 0.0 & 2.8 & 16.5 & 0.0 & 5.2 & 1.3 \\
\hline 41 & 393807106481501 & $06 / 20 / 07$ & 10.9 & 6,733 & 0.7 & 24.2 & 0.0 & 5.0 & 23.7 & 0.0 & 7.0 & 8.6 \\
\hline 42 & 393813106490201 & $06 / 10 / 07$ & 9.4 & 6,664 & 0.6 & 18.9 & 0.0 & 4.3 & 17.7 & 0.0 & 3.9 & 1.4 \\
\hline 43 & 393923106504801 & $05 / 17 / 07$ & 10.5 & 6,707 & 0.6 & 30.3 & 0.0 & 7.8 & 17.6 & 0.0 & 6.1 & 2.2 \\
\hline 44 & 393907106505201 & $05 / 17 / 07$ & 11.1 & 6,556 & 0.5 & 37.7 & 0.0 & 6.9 & 14.4 & 0.0 & 10.2 & 0.3 \\
\hline 45 & 393850106511701 & $08 / 23 / 06$ & 10.3 & 6,500 & 0.6 & 24.1 & trace & 0.2 & 18.2 & 1.0 & 11.9 & 3.5 \\
\hline 47 & 393917106512501 & $08 / 22 / 06$ & 13.6 & 6,650 & 0.5 & 8.9 & 0.0 & 3.3 & 15.0 & 0.0 & 13.1 & 1.7 \\
\hline 49 & 393847106520401 & $05 / 17 / 07$ & 11.2 & 6,531 & 0.6 & 51.0 & 0.0 & 6.2 & 15.3 & 0.0 & 9.7 & 1.0 \\
\hline 50 & 393903106521901 & $08 / 22 / 06$ & 12.8 & 6,460 & 0.6 & 23.9 & 0.0 & 3.2 & 17.5 & 0.0 & 9.3 & 3.0 \\
\hline 52 & 393702106545401 & $08 / 23 / 06$ & 9.6 & 6,722 & 0.6 & 33.5 & 0.0 & 7.0 & 16.7 & 0.0 & 6.9 & 1.6 \\
\hline 53 & 393740106545801 & $05 / 22 / 07$ & 9.6 & 6,644 & 0.6 & 34.4 & trace & 0.3 & 17.3 & 0.0 & 6.9 & 2.2 \\
\hline 55 & 393832106561101 & $05 / 19 / 07$ & 11.4 & 6,418 & 0.6 & 44.3 & 0.0 & 5.3 & 17.9 & 0.0 & 9.0 & 3.3 \\
\hline 57 & 393845106563401 & $08 / 22 / 06$ & 12.5 & 6,350 & 0.7 & 40.9 & 0.0 & 3.9 & 20.0 & 0.0 & 8.5 & 5.2 \\
\hline 59 & 393632106581101 & $08 / 23 / 06$ & 13.5 & 6,620 & 0.7 & 14.5 & 0.0 & 5.7 & 19.5 & 0.0 & 4.9 & 3.5 \\
\hline 60 & 393658106574101 & $05 / 20 / 07$ & 8.5 & 6,528 & 0.7 & 18.7 & 0.0 & 3.9 & 18.5 & 0.0 & 1.8 & 1.2 \\
\hline 61 & 393759106565701 & $06 / 07 / 07$ & 11.6 & 6,446 & 0.5 & 43.4 & 0.0 & 5.6 & 14.1 & 0.0 & 9.7 & -0.2 \\
\hline 62 & 393845107022101 & $08 / 25 / 06$ & 15.1 & 6,250 & 0.6 & 10.3 & 0.5 & 0.2 & 18.8 & 2.0 & 11.4 & 2.9 \\
\hline 63 & 393900107030701 & $05 / 19 / 07$ & 12.4 & 6,152 & 0.6 & 28.0 & 0.0 & 0.3 & 19.2 & 2.5 & 11.2 & 2.7 \\
\hline
\end{tabular}


EAST HALF OF THE EAGLE RIVER WATERSHED VALLEY-FILL AQUIFER

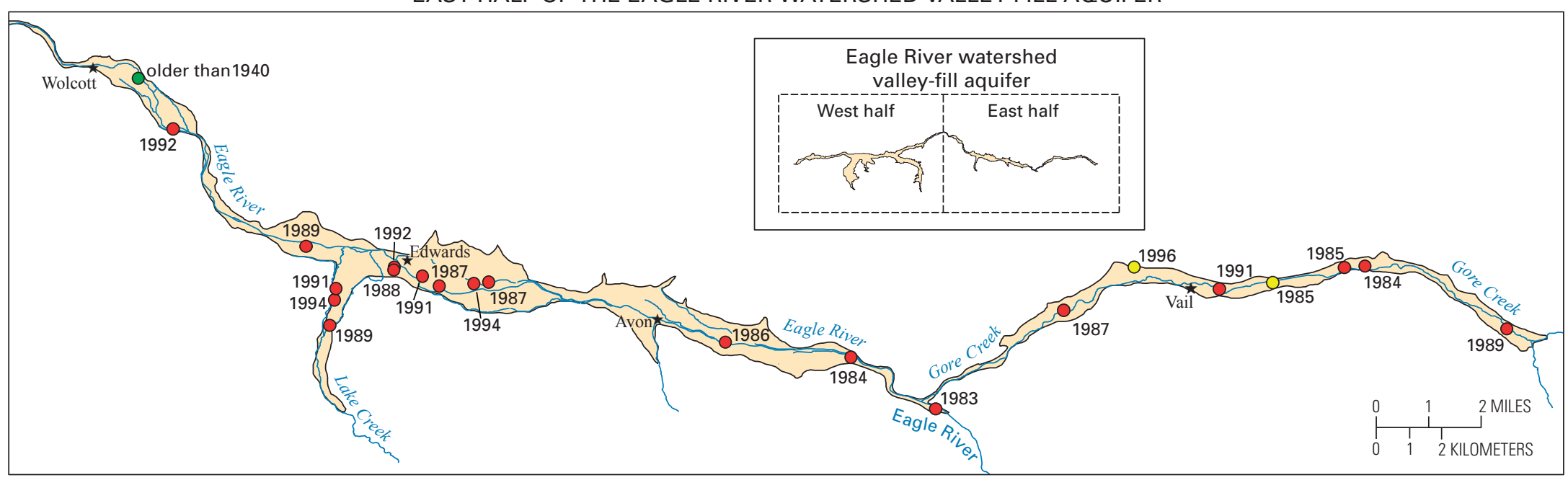

WEST HALF OF THE EAGLE RIVER WATERSHED VALLEY-FILL AQUIFER

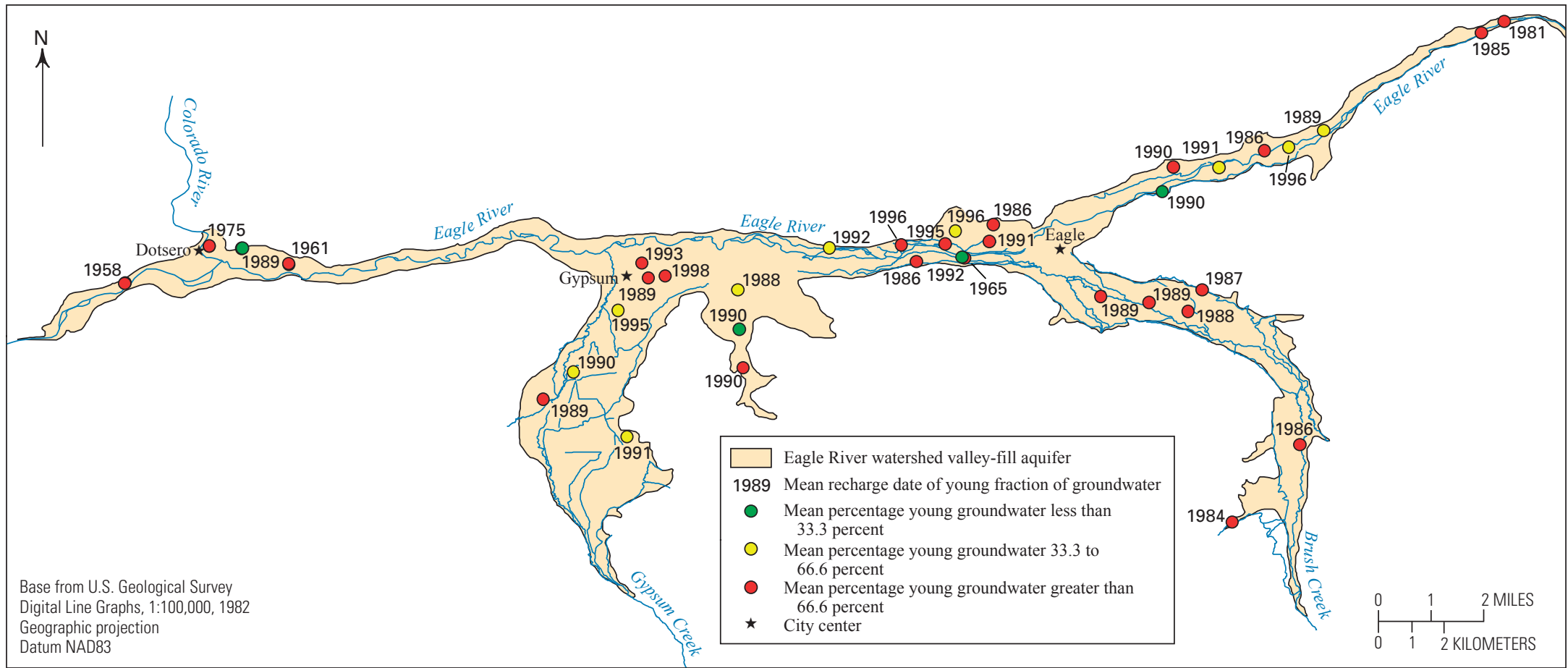

Figure 13. Mean recharge date of the young fraction of groundwater and the percentage of young groundwater from wells completed in the Eagle River watershed valley-fill aquifer, 2006-2007, Eagle County, Colorado. 
CFCs in precipitation is about 550 parts per trillion (ppt) (International Atomic Energy Agency, 2006), and the largest concentration of CFC-12 measured in air samples was 540 ppt (table 11), but 40 out of 59 wells sampled in the Eagle River watershed had concentrations greater than $550 \mathrm{ppt}$ (table 3). CFC-12 was used as coolants in air-conditioning units and refrigeration, blowing agents in foams, insulation, and packing materials, propellants in aerosol cans, and as solvents (Plummer and Busenberg, 1999). It is possible that the elevated CFC-12 concentrations in groundwater in the Eagle River watershed are from a combination of point and nonpoint sources of contamination. The point sources could include spills of CFC-12 containing solvents on the ground. Nonpoint sources could include the discharge of treated wastewater that contains CFC-12 to the Eagle River, which is subsequently diverted by the irrigation networks and reapplied to the land surface. Although CFC-12 could not be used for groundwater age determination, the anomalously high CFC-12 concentrations could be a useful indicator of areas with a high predisposition to anthropogenic contamination in groundwater.

\section{Low-Level Concentrations of Volatile Organic Compounds}

VOCs were detected in all water samples using the lowlevel minimum detection levels (fig. 14). The low-level VOCs were analyzed from the same samples, and at the same time, using the same analytical equipment as the CFC samples. Five replicate $\mathrm{CFC} / \mathrm{VOC}$ samples were collected at each site, and in most cases three of those samples were analyzed for each site and the concentrations were averaged. In cases where variability in concentrations was high between each replicate sample, additional samples were analyzed for each site. Analyzing replicate samples at each site helps to quantify the variability of analytical results at these extremely low concentrations. The values listed in table 4 are mean values from the multiple replicate samples analyzed.

Detection of halogenated VOCs in groundwater can be attributed to three general categories of source: (1) natural, atmospheric origin; (2) anthropogenic sources in the atmosphere; and (3) contaminant anthropogenic sources (Plummer and others, 2008). Methyl iodide (and methyl bromide) is detected in air and produced naturally by marine and aquatic biological processes (Sturges and others, 2001; Bell and others, 2002; Cox and others, 2005; Chuck and others, 2005). Approximately 90 percent of global emissions of chloroform is estimated to be of natural origin from sources such as volcanic gases (Isidorov and others, 1990), marine algae (Gribble, 1994; Laturnus and others, 2002), and soil fungi (McCulloch, 2003). Although most of the chloroform of natural origin is oxidized (to hydrogen chloride and carbon dioxide) in the atmosphere, some low-level detections of chloroform in rural groundwater may be of natural, terrestrial origin (McCulloch, 2003). Although a natural substance, salt concentrations $(\mathrm{NaCl}$ and $\mathrm{CaCl}_{2}$ ) have increased in the environment because of the use of these compounds for roadway deicing. A recent study (Princeton Geoscience, 2005) indicated that the abundance of chloride ions in the runoff of deicing meltwaters may be enhancing the natural chloroform formation process in nearby soils. Natural emissions of other naturally occurring halogenated VOCs have been identified, including methyl chloride, tetrachloroethene, trichloroethene, and methylene chloride (Keene and others, 1999; Khalil and others, 1999), with 5 percent of the total 1998 global input of tetrachloroethene and 10 percent of that of trichloroethene attributed to natural, oceanic sources (Keene and others, 1999). Marine algae also have been identified as natural sources for bromodichloromethane, dibromochloromethane, and bromoform (Gribble, 1994).

Most of the halogenated VOCs in the atmosphere also have anthropogenic sources. For example, concentrations of chlorofluorocarbons in the atmosphere are likely entirely of anthropogenic origin, and most of the VOCs that have natural sources also can be attributed to various industrial sources. Samples containing VOCs of contaminant anthropogenic origin are of greatest concern because they represent waters that are being affected by contaminant sources in addition to atmospheric sources. By defining pre-anthropogenic and modern anthropogenic atmospheric concentrations of halogenated VOCs in groundwater, it is possible to identify those samples that exceed this threshold and thus contain anthropogenic sources of VOCs (Plummer and others, 2008).

A means of identifying samples that are being affected by contaminant sources of halogenated VOCs is to plot the sum of the total concentrations of dissolved halogenated VOCs

Table 11. Chlorofluorocarbon and volatile organic compounds analyzed from air samples collected in the Eagle River watershed, 2006-2007, Eagle County, Colorado.

[CFC, chlorofluorocarbon; m, month; d, day; y, year; ppt, parts per trillion; --, not analyzed]

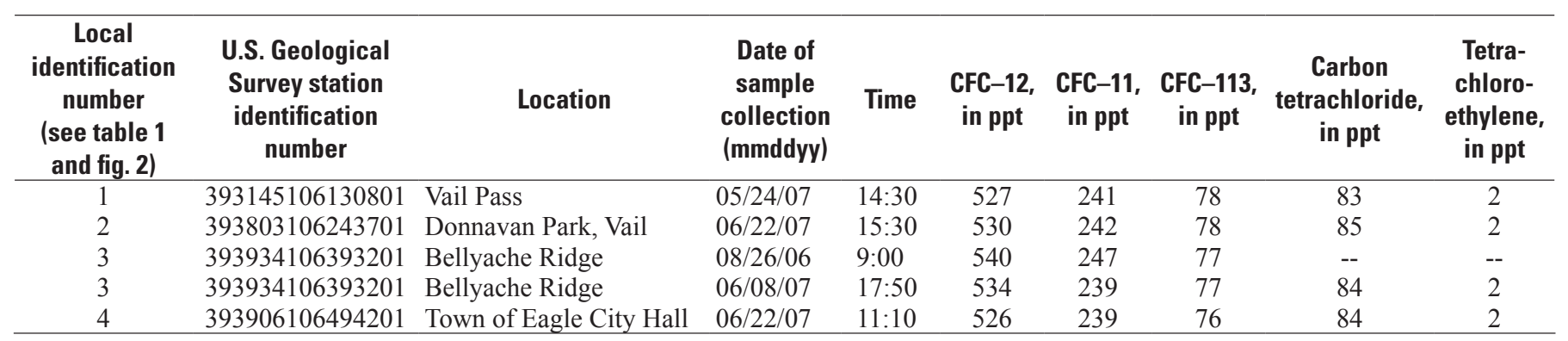




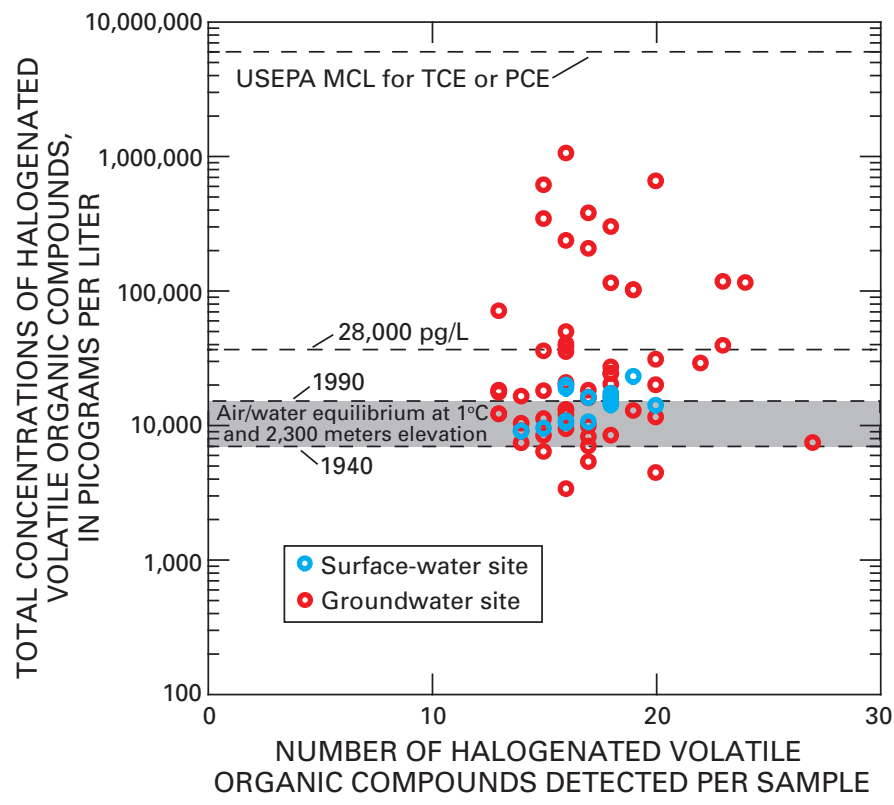

Figure 14. Relations between total concentrations of halogenated volatile organic compounds with the total number of halogenated volatile organic compounds, Eagle River watershed, 2006-2007, Eagle County, Colorado.

(TDVOC) in the sample as a function of the total number of VOC compounds detected per sample (Plummer and others, 2008) (fig. 14). Samples that are being affected by contaminant sources of halogenated VOCs have larger concentrations than concentrations of halogenated VOCs in groundwater that are only of atmospheric origin. Twenty-five halogenated VOCs were analyzed in this report. Table 4 lists concentrations of the 15 most commonly detected VOC compounds by this study and the TDVOC of those 15 compounds added together. It has been shown that TDVOC computed for these 15 selected compounds accounts for, on average, more than 95 percent of the total concentration of halogenated VOCs detected in groundwater samples (Plummer and others, 2008), as was the case for the Eagle County samples. The remaining 10 compounds were detected at very low concentrations that contribute an average of only about 5 percent to the value of TDVOC concentration in the samples. It is important to note the very low concentrations of VOCs in groundwater and surface water of the ERWVFA; all concentrations were at least one order of magnitude less than the USEPA Maximum Contaminant Level for tricholoroethene or tetrachloroethene (fig. 14).

Values of TDVOC were calculated from historical atmospheric concentrations of VOCs using Henry's law solubility data, as in Plummer and others (2008). The air/water equilibrium was calculated for 1940 (before significant anthropogenic input of VOCs to the atmosphere) and for 1990 (the estimated maximum concentration of VOCs in the atmosphere from natural and anthropogenic sources combined) at the mean annual temperature of $1^{\circ} \mathrm{C}\left(34^{\circ} \mathrm{F}\right)$ and at $2,300 \mathrm{~m}(7,500 \mathrm{ft})$ of elevation for the Eagle River watershed. Total concentrations of halogenated VOCs detected in surface water in the Eagle River watershed plot near values of the air/water equilibrium of water with the modern atmosphere calculated at the elevation and mean annual temperatures expected in the Eagle River watershed (fig. 14). The surface-water samples were collected from each site during high flow (snowmelt runoff) and low flow (late summer) to bracket seasonal fluctuations (table 4). Sites 393737106165900 (Gore Creek Below Black Gore Creek), 09068000 (Brush Creek Near Eagle), and 09069500 (Gypsum Creek Near Gypsum) are located upgradient from any substantial anthropogenic sources of VOCs, yet water from those sites had substantial TDVOC concentrations, confirming that substantial concentrations of TDVOCs in surface water can be from natural and anthropogenic atmospheric sources in the Eagle River watershed. The highest TDVOC concentrations at all surface-water sites were during high-flow conditions (May); the average TDVOC concentration for the high-flow (May) surface-water samples was about $18,000 \mathrm{pg} / \mathrm{L}$. The average TDVOC concentration for low-flow (August) samples was about 12,000 pg/L. Site 393834106230401 is located on Gore Creek immediately upgradient from a wastewater-treatment plant, and site 393810106241501 is located immediately downstream from the same wastewater-treatment plant. Chloroform concentrations were higher in Gore Creek downstream from the wastewater-treatment plant, but most other VOC concentrations were lower downstream from the wastewater-treatment plant. Elevated chloroform concentrations are expected in wastewater because of the presence of chlorinated water, free chlorine, and elevated organic carbon (Carter and others, 2008; Plummer and others, 2008).

The maximum TDVOC concentration detected in surfacewater samples was $22,880 \mathrm{pg} / \mathrm{L}$, and the standard deviation of all TDVOC concentrations in surface water was 4,206 pg/L 
(table 4). Adding the maximum observed TDVOC concentration in surface water with the standard deviation, and then rounding up, produced a maximum likely TDVOC concentration in surface water in the Eagle River watershed of about $28,000 \mathrm{pg} / \mathrm{L}$. Water from wells with TDVOC concentrations greater than $28,000 \mathrm{pg} / \mathrm{L}$ probably have been affected by anthropogenic contamination. Twenty-one of the 61 groundwater samples had TDVOC values that exceeded $28,000 \mathrm{pg} / \mathrm{L}$, indicating those waters have been affected by VOCs from anthropogenic sources. Low-level detections of VOCs can provide a measure of the predisposition of an aquifer to anthropogenic contamination (Shapiro and others, 2003; Plummer and others, 2008; Carter and others, 2008). The locations of wells with TDVOC concentrations greater than $28,000 \mathrm{pg} / \mathrm{L}$ were used to identify areas with a large predisposition to contamination by VOCs in one of the logistic regression models described in the next section of this report.

\section{Groundwater Probability Modeling}

Logistic regression statistical modeling techniques were used to develop three statistical models that predict the probability of groundwater contamination by various contaminants. These three models used different compounds such as nitrate and VOCs to provide an indication of the probability of groundwater contamination under a variety of conditions and contaminant inputs. These three models are the probability of elevated nitrate concentrations, the probability of unmixed young water (using CFC-11 concentrations and tritium activities), and the probability of elevated VOC concentrations.

Before using the groundwater probability maps, the results of the groundwater age dating should be recognized. The median groundwater recharge date was 1989, indicating that most groundwater in the ERWVFA was young water (younger than 1940) and had a high predisposition to contamination. The probability maps showed areas that have a greater predisposition to contamination by anthropogenic compounds than other areas, but just about all areas had a high predisposition to contamination because very few wells in the ERWVFA had large portions of water older than 1940 (table 3).

\section{Development of Nitrate Model}

A logistic regression model was developed to predict the probability of nitrate concentrations above $1 \mathrm{mg} / \mathrm{L}$ in groundwater (table 12, fig. 15), which is the maximum concentration expected in natural recharge water in the Eagle River watershed. The nitrate probability model was developed because groundwater contamination from fertilizers, manure, and wastewater from sources such as conventional domestic septic systems is a concern in Eagle County. Depth to groundwater, clay content, soil drainage, depth to groundwater in the soil layer, soil liquid limit, soil organic-matter content, soil sand content, and soil slope were significant variables in the nitrate model (table 12). Overall performance of the model was good, with a McFadden's rho-squared value of 0.490 , and total correct predictions of 0.778 .

The nitrate model predicted a lower probability of elevated nitrate concentrations in areas with shallower depth to groundwater and more transmissive soils, which is opposite to relations observed in other logistic regression models (Rupert, 1998; Nolan and others, 2002; Gurdak and Qi, 2006). This may be due to two reasons. The first is that some of the nitrate may be naturally occurring. As the result of evapotranspiration, nitrate can naturally accumulate in soils in arid environments (such as the environment near Gypsum and Dotsero), which leaves chloride and nitrate salts that can leach to groundwater following changes in land use or climate (Walvoord and others, 2003). Naturally occurring nitrate may be concentrated in areas with deeper depth to water and less transmissive soils. The second reason is that the areas with shallow groundwater and more transmissive soils are located near surface-water bodies such as the Eagle River. The shallow groundwater has more immediate recharge from the surface-water bodies, which provide recharge water with nitrate concentrations similar to precipitation that may dilute nitrate concentrations in groundwater to less than $1 \mathrm{mg} / \mathrm{L}$. Nitrate in groundwater in areas with larger depth to groundwater and less transmissive soils would have less opportunity for dilution by surface-water recharge. Nitrate supplied by fertilizers, manure, or wastewater in areas of large depth to groundwater and less transmissive soils may tend to concentrate in the groundwater because of the lower amount of recharge available to dilute the nitrate. Areas such as the Brush Creek and Gypsum Creek valleys (fig. 15) have low nitrate probability ratings. Future urban development may reduce the amount of recharge from irrigation in the valley bottoms, which may increase the depth to groundwater. This may, in turn, increase the probability of nitrate contamination.

\section{Development of Chlorofluorocarbon-11 and Tritium Model}

Plotting CFC-11 concentrations with tritium activities provided an indication of water that was composed of mostly young water or contained a substantial fraction of old water (fig. 12). Points that lie close to the piston-flow line, which is the line of measured CFC-11 concentration in parts per trillion and tritium in precipitation decayed to the year 2007 through time, were composed of 100 percent young water (water younger than 1940). Points plotting below the pistonflow line were composed of a substantial portion of old water (water older than 1940), or are waters recharged through deep unsaturated zones. Water composed of 100 percent young water could have a higher probability of anthropogenic contamination at concentrations of concern than water with a portion of old water.

To construct a logistic regression model predicting the probability of unmixed young water (younger than 1940) using CFC-11 and tritium (fig. 16), the data set was coded as zero for 
Table 12. Logistic regression modeling results, coefficients, and individual p-values of independent variables significantly related with the probability of nitrate concentrations greater than 1 milligram per liter, the probability of unmixed young water (using chlorofluorocarbon-11 concentrations and tritium activities), and the probability of total volatile organic compounds greater than 28,000 picograms per liter in the Eagle River watershed valley-fill aquifer, Eagle County, Colorado.

[CFC, chlorofluorocarbon; TDVOCs, total volatile organic compound concentrations; mg/L, milligrams per liter; pg/L, picograms per liter; --, no relation observed; sensitivity, the number of correctly predicted events divided by the total number of observed events; specificity, the number of correctly predicted reference events divided by the total number of observed reference events; total correct predictions, the number of correctly predicted events plus the number of correctly predicted reference events divided by the total number of all events; values not enclosed in parentheses are logistic regression coefficients; values enclosed in parentheses are individual p-values; independent variables in bold are used in equation 1 for the logistic regression models; SSURGO, Soil Survey Geographic Database; STATSGO, State Soil Geographic Database]

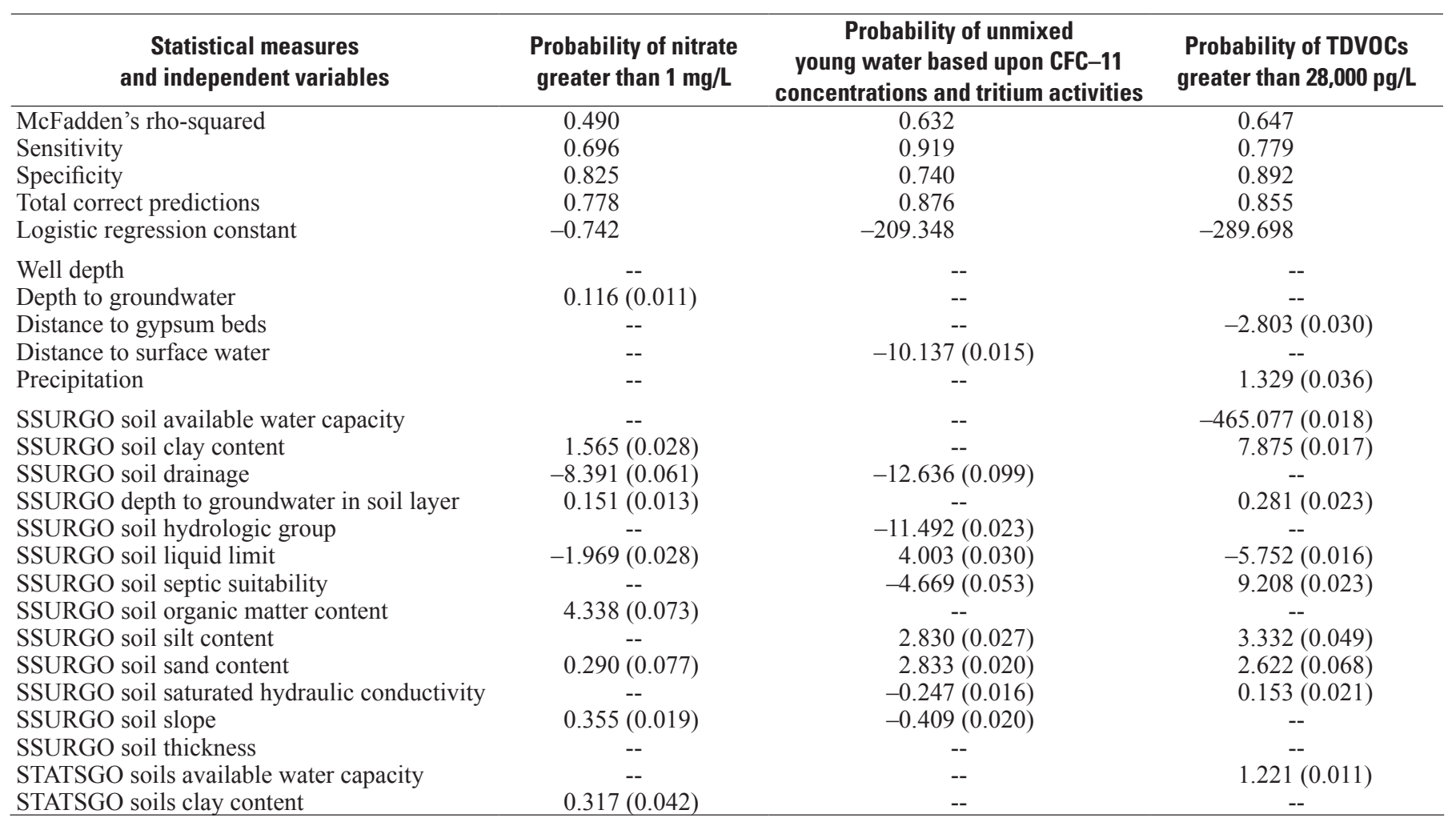

water containing a substantial portion of old water, and one for water composed of 100 percent young water. Water from 12 wells had CFC-11 concentrations greater than 250 ppt (table 3), which is the maximum concentration measured in air samples (table 11). Those 12 wells also were coded as one because they contain elevated concentrations of CFC-11 and have a higher probability of anthropogenic contamination. The resulting logistic regression model had a large McFadden's rho-squared (0.632), a large sensitivity (0.919), and a large total correct predictions $(0.876)$ (table 12), indicating an effective model. Independent variables included in the model were distance to surface water, soil drainage, soil hydrologic group, soil liquid limit, soil septic suitability, soil silt content, soil sand content, soil saturated hydraulic conductivity, and soil slope (table 12).

The percentage of young water is an indicator of the predisposition of groundwater to anthropogenic contamination because groundwater composed of 100 percent young water has a greater likelihood of recharge by water containing anthropogenic compounds than water containing a substantial portion of old (older than 1940) water. Most compounds likely to cause concern in the Eagle River watershed are anthropogenic in origin that were produced mostly during the past 50 years; water composed of 100 percent young water has the greatest likelihood to contain anthropogenic compounds. Even if the young portion of water contains compounds of concern, those concentrations will be diluted by the older (uncontaminated) water, reducing the overall concentrations. For an overall measure of the predisposition to anthropogenic groundwater contamination, this probability model is probably the most useful out of the three probability models developed.

\section{Development of Volatile Organic Compound Model}

A model was developed to predict the probability of total dissolved VOC concentrations (TDVOC) in groundwater greater than $28,000 \mathrm{pg} / \mathrm{L}$ (table 12, fig. 17). Significant independent variables were distance to gypsum beds, precipitation, soil available water capacity, soil clay content, depth to groundwater in soil layer, soil liquid limit, soil septic suitability, soil silt content, 


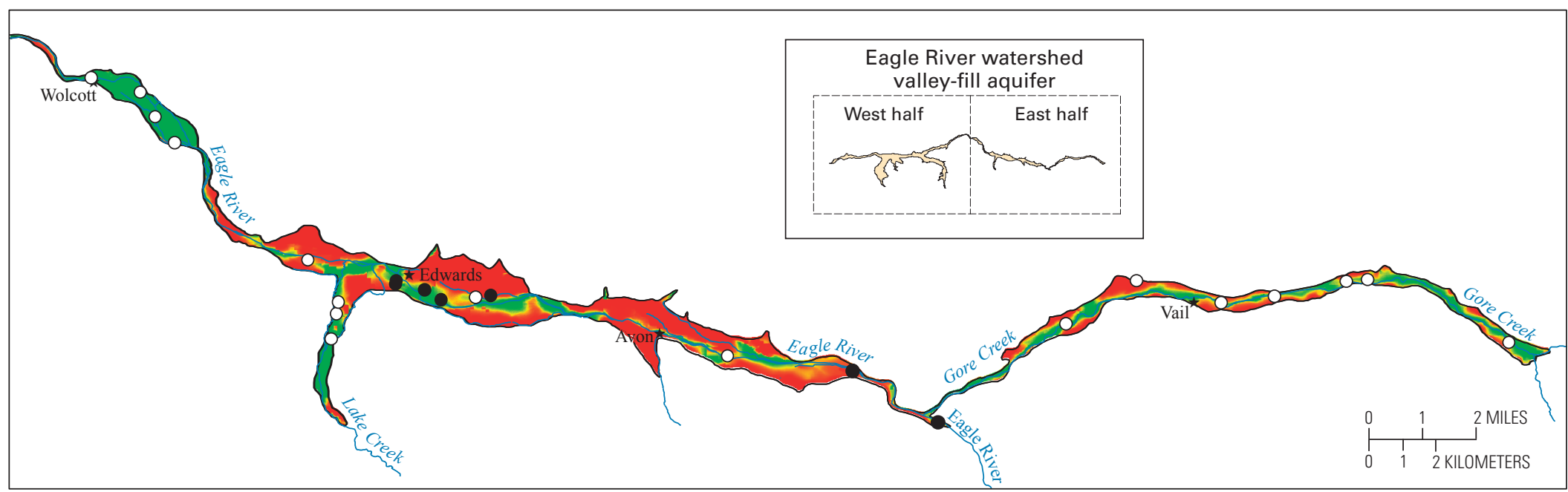

WEST HALF OFTHE EAGLE RIVER WATERSHED VALLEY-FILL AQUIFER

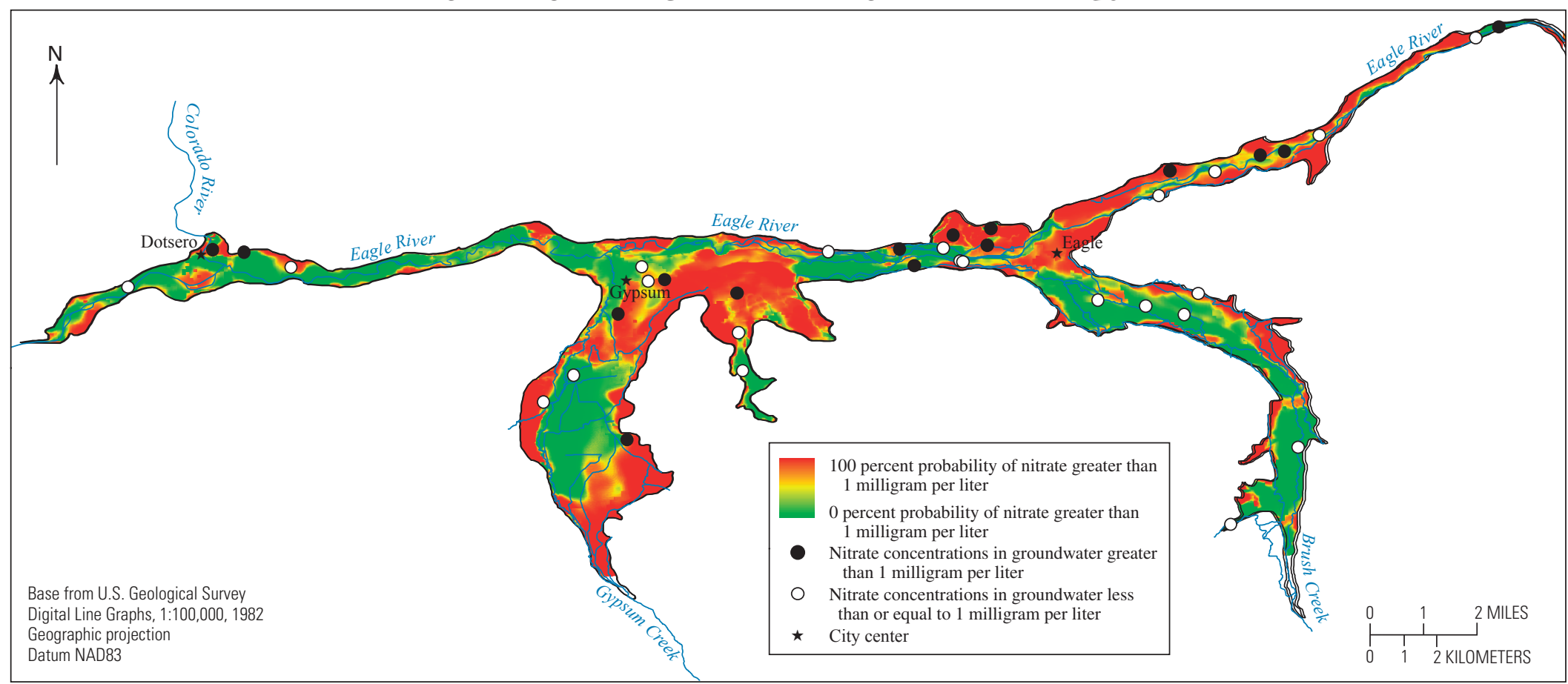

Figure 15. Probability of detecting nitrate concentrations greater than 1 milligram per liter in the Eagle River watershed valley-fill aquifer, 2006-2007, Eagle County, Colorado. 
EAST HALF OF THE EAGLE RIVER WATERSHED VALLEY-FILL AQUIFER

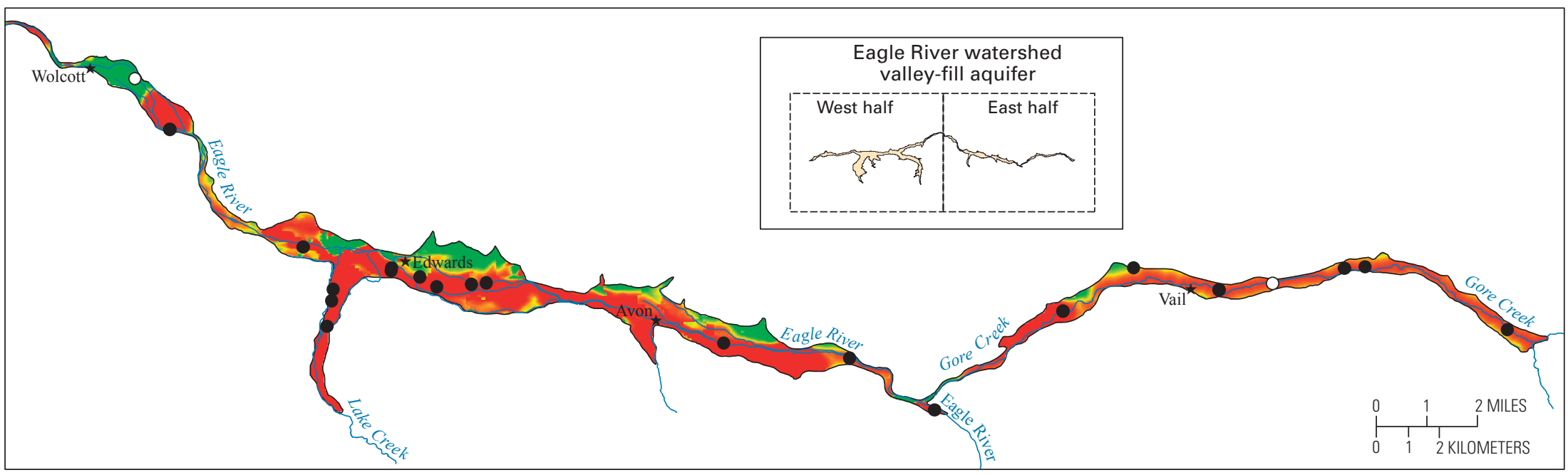

WEST HALF OF THE EAGLE RIVER WATERSHED VALLEY-FILL AQUIFER

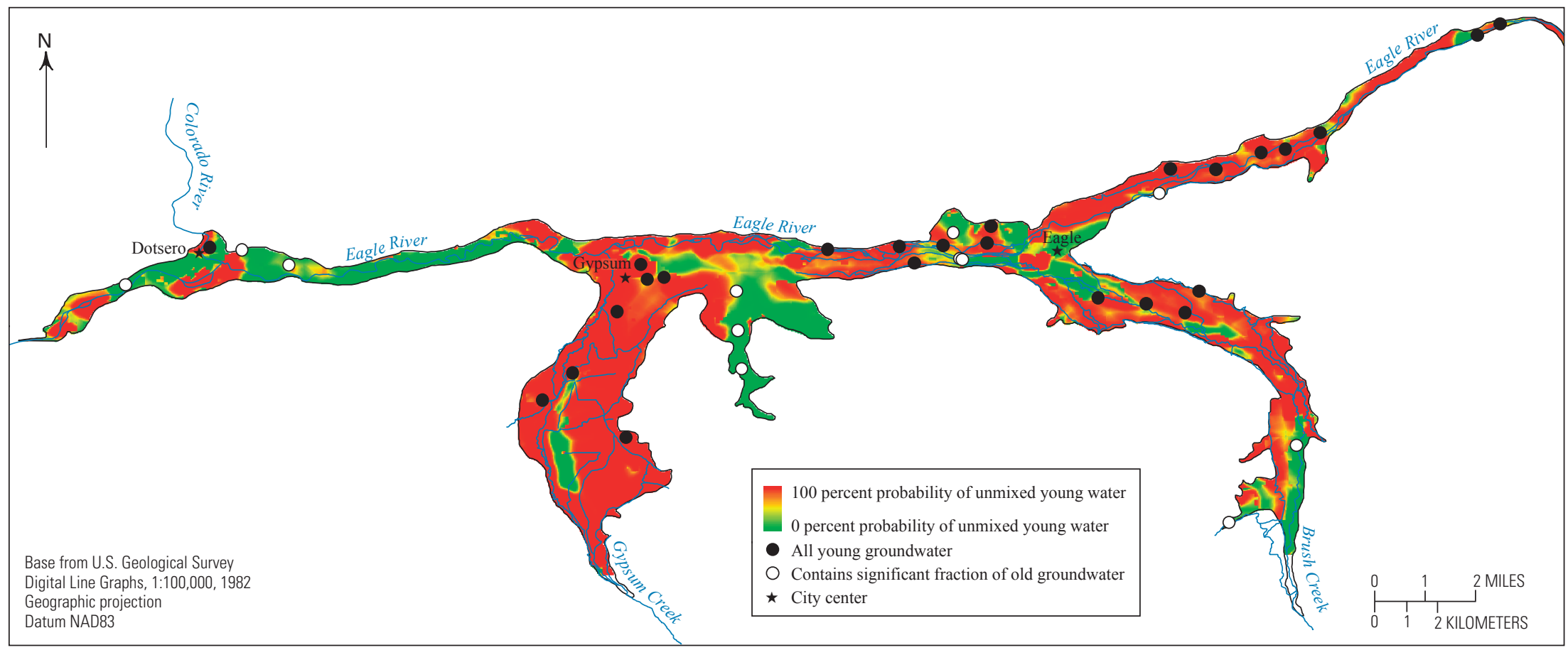

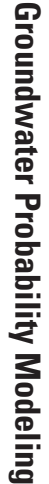

Figure 16. Probability of detecting unmixed young water in the Eagle River watershed valley-fill aquifer (using chlorofluorocarbon-11 concentrations and tritium activities), 2006-2007, Eagle County, Colorado. 


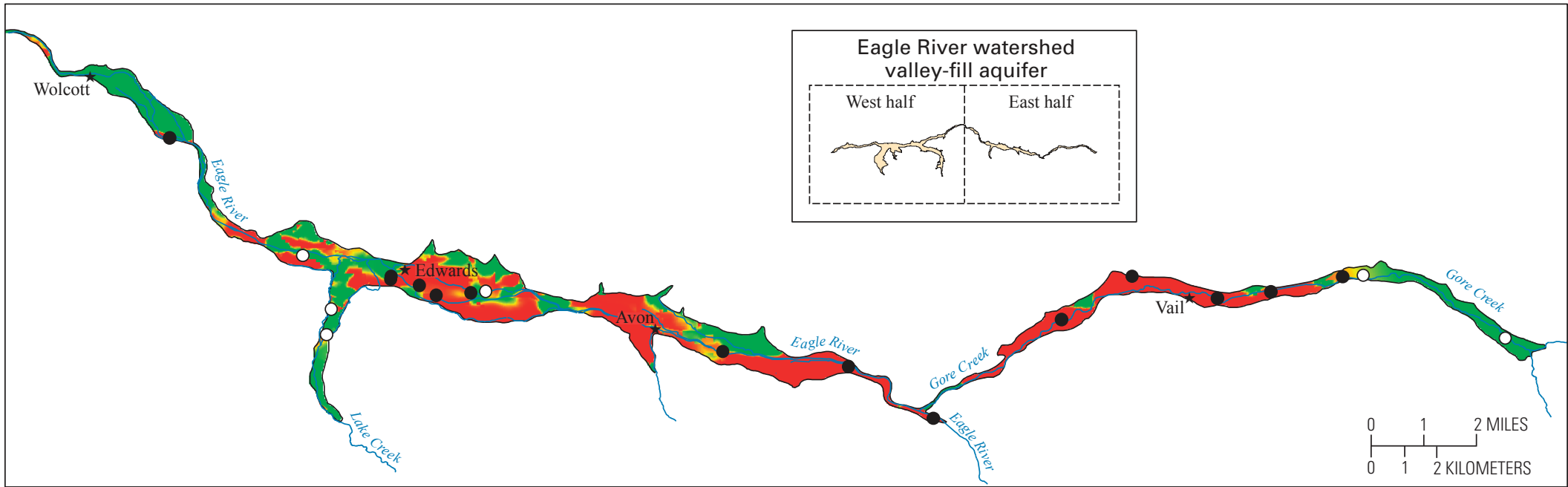

WEST HALF OF THE EAGLE RIVER WATERSHED VALLEY-FILL AQUIFER

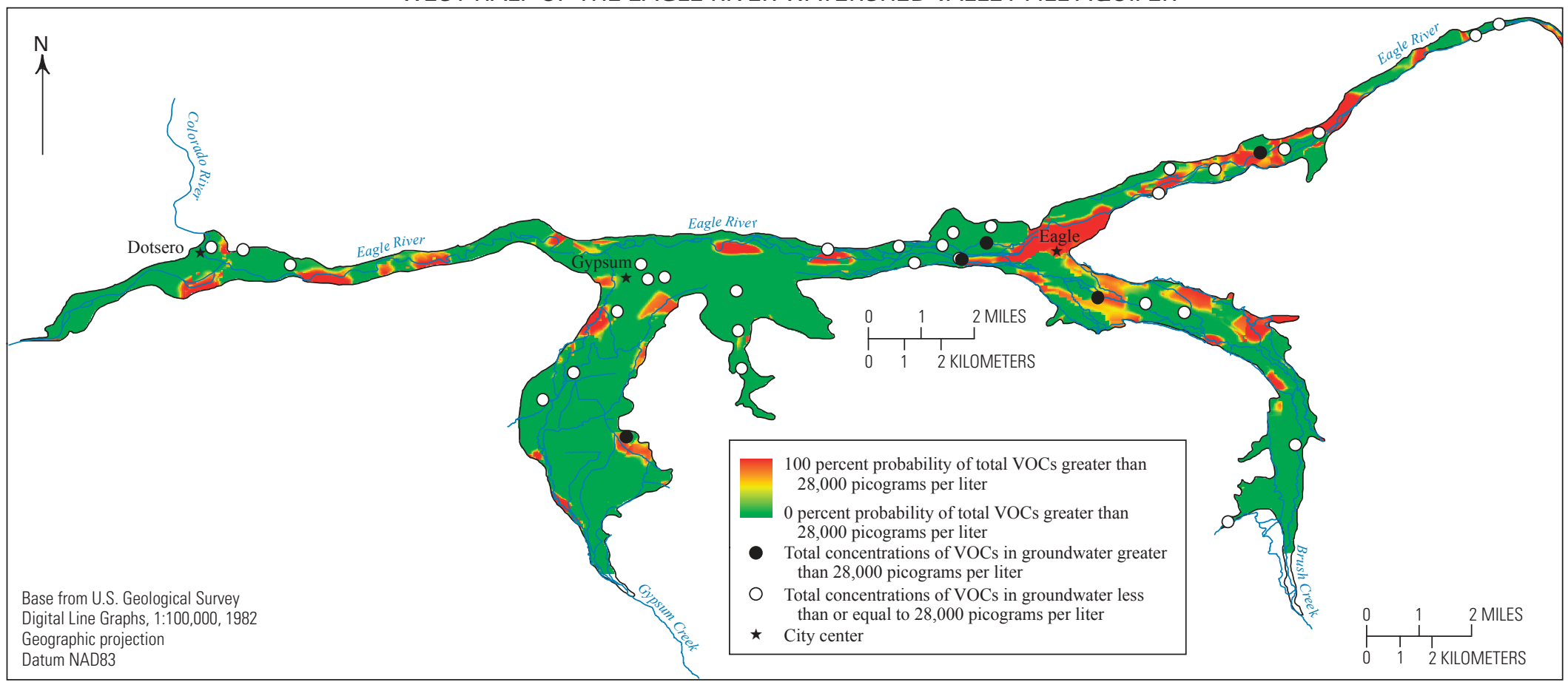

Figure 17. Probability of detecting total volatile organic compound concentrations greater than 28,000 picograms per liter in the Eagle River watershed valleyfill aquifer, 2006-2007, Eagle County, Colorado. 
soil sand content, and soil saturated hydraulic conductivity. The TDVOC model was one of the most significant probability models produced (table 12). McFadden's rho-squared was 0.647 , the sensitivity was 0.779 , and the total correct predictions was 0.855 .

Most wells with elevated TDVOC concentrations are located in the eastern portion of the ERWVFA (fig. 17), presumably because this is where the most intensive urban development has occurred. The TDVOC detections may be an indicator of light industrial and construction uses of solvents such as PCE, or the result of spills.

\section{Verification of Probability Models}

Previous probability mapping projects in other areas have validated the performance of the models by comparing them to an independent set of groundwater quality data (Rupert, 1998; Rupert, 2003; Gurdak and Qi, 2006). Unfortunately, an independent set of groundwater quality data is not available for the Eagle County watershed. To verify the models, the percentage of actual detections was plotted with the predicted probability of detections using a deciles of risk calculation. R-squared values of plots of actual detections with the predicted probability of detections were between 0.995 and 0.998 , verifying they are highly effective models.

\section{Comparison of Probability Models}

The results of the groundwater age dating indicated that most areas in the ERWVFA had a high predisposition to contamination because most waters had groundwater recharge dates younger than 1980 . The three probability models further delineated the probability of contamination for three different classes of compounds.

The nitrate probability model (fig. 15) showed the relative risk of elevated nitrate concentrations in groundwater from sources of nitrate such as fertilizers, manure, and wastewater. Based upon the young groundwater ages, the entire ERWVFA had a high predisposition to nitrate contamination from fertilizers, manure, and wastewater, but areas with larger depth to groundwater and less transmissive soils had a greater risk. Wells with nitrate concentrations greater than $1 \mathrm{mg} / \mathrm{L}$ in 2006-07 are shown in figure 10. Areas such as the Brush Creek and the central part of Gypsum Creek valleys (fig. 15) had low nitrate probability ratings. Future urban development may reduce the amount of recharge from irrigation in the Brush Creek and Gypsum Creek valley bottoms, which may increase the depth to groundwater, hence increasing the probability of nitrate contamination.

The model predicting the probability of unmixed young water in the ERWVFA (fig. 16) can be considered a generic model that is applicable for most contaminants. If a spill were to occur on the land surface or into the local surface water, those contaminants could be transported to the groundwater along the same pathways that the young recharge water uses. Although all areas of the ERWVFA had a high predisposition to contamination, areas with a high probability of unmixed young water (fig. 16) had the highest probability of carrying contamination with those young recharge waters into the groundwater.

Based upon the high occurrence of VOC detections observed by this study, VOC contamination is a concern in the ERWVFA. Although the entire ERWVFA had a predisposition to VOC contamination to some degree, the VOC probability map (fig. 17) indicated that the areas near Vail, Avon, and Edwards had the highest probability of groundwater contamination if a spill should occur.

\section{Appropriate Uses of the Probability Maps}

The probability models and maps are designed to portray the likelihood of groundwater in the ERWVFA to be affected by anthropogenic contamination. These models and maps do not show areas that are actually (currently) contaminated; rather, they show the areas that have a high likelihood for being contaminated if a compound of concern were released to the environment in the terms of percent probability. Probability is a statistical measure of how likely an event will occur. Probability is not the same as certainty. A well in a high-probability area is not necessarily contaminated because contamination also can depend on the type of contaminant released, how much of that contaminant was released, and other factors not taken into account by the logistic regression models. Although the probability maps show predictions of detections as a percent probability, there is inherent uncertainty within these predictions that is not shown in the probability map.

The probability models and maps are intended to be a first approximation at developing a consistent rating method for the entire study area and may have several limitations for use at the site or field scale. The models and maps do not account for local point sources of contaminants or features and processes that may promote focused recharge, preferential groundwater flow, or bypass mechanisms. Additional site-specific data are needed before site-specific decisions are made, such as the site-specific design of a wastewater-disposal system.

The probability maps should not be used at a scale any larger than 1:24,000, which is the scale of the SSURGO soils data. Some site-specific variables, such as improper well construction and local spills of contaminants, were not accounted for in the models. The Eagle River watershed is rapidly being developed. As development activities continue, some variables such as surficial soils and irrigation networks may be altered through construction activities, which may have unpredictable effects on the groundwater probability ratings. 

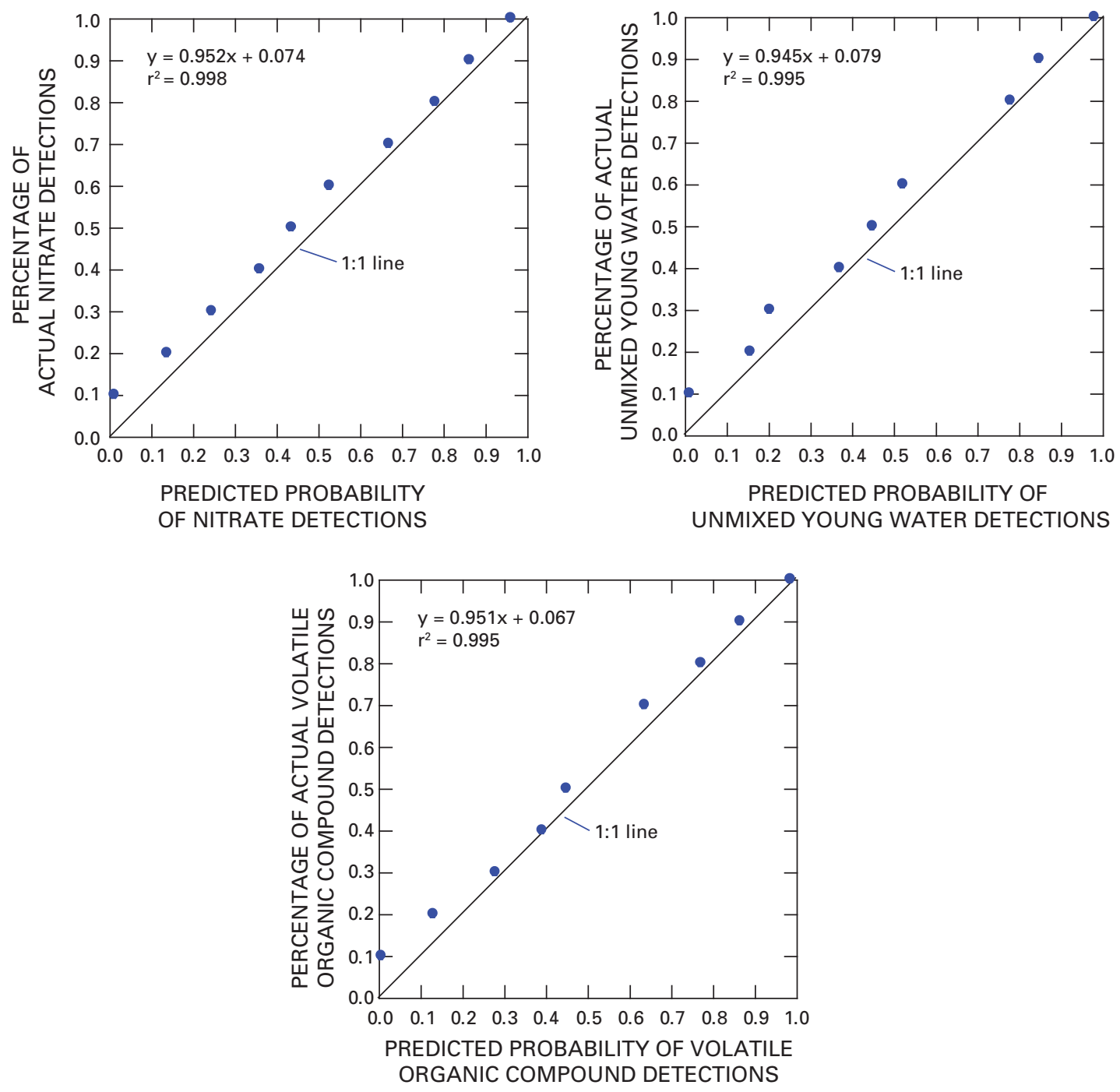

Figure 18. Percentage of actual detections of nitrate greater than 1 milligram per liter, detections of unmixed young water, and detections of total volatile organic compounds greater than 28,000 picograms per liter, plotted with the predicted probability of detections of those compounds, Eagle River watershed valley-fill aquifer, Eagle County, Colorado.

\section{Summary}

The Eagle River watershed is near the destination resort town of Vail, Colorado. The area has a fast growing permanent population, and the resort industry is rapidly expanding. The popularity of the area is largely due to the presence of high-value ecosystems and relatively pristine environmental conditions that the community wishes to protect. A large percentage of the land undergoing development is located above the Eagle River watershed valley-fill aquifer (ERWVFA), which has a high predisposition to groundwater contamination. As development continues, local organizations need tools to evaluate potential land-development effects on ground- and surface-water resources so that wise land-use decisions can be made. To help develop these tools, the U.S. Geological Survey (USGS), in cooperation with Eagle County, the Eagle River Water and Sanitation District, the Town of Eagle, the Town of Gypsum, and the Upper Eagle Regional Water Authority, conducted a study in 2006-2007 of the groundwater quality, age, and probability of contamination in the ERWVFA, north-central Colorado.

The overall purpose of this report was to develop maps that show the predisposition of the ERWVFA to contamination by anthropogenic compounds (contamination caused or produced by humans). Logistic regression statistical modeling techniques were applied to measured concentrations of environmental tracers (nitrate, tritium, chlorofluorocarbons (CFCs), and halogenated volatile organic compounds [VOCs]) in groundwater to develop statistical models that predict the probability of groundwater contamination by anthropogenic compounds in the ERWVFA. Previous groundwater probability mapping projects in other areas of the country have used agricultural contaminants such as atrazine to calibrate the statistical models. Nitrate, tritium, CFCs, and VOCs were used to calibrate 
the statistical models because development in Eagle County is urban and not agricultural, and development has occurred, for the most part, during the past 20 years or so.

Groundwater-quality samples were collected once from 10 sites during August 2006 and once from an additional 51 sites during May through June 2007 and analyzed for major ions, nutrients, stable isotopes of hydrogen and oxygen in water $\left({ }^{2} \mathrm{H}\right.$ and $\left.{ }^{18} \mathrm{O}\right)$, tritium, dissolved gases, $\mathrm{CFCs}$, and VOCs determined with very low-level laboratory methods. Surface-waterquality samples were collected quarterly from six sites between October 2006 and September 2007 and analyzed for major ions, nutrients, ${ }^{2} \mathrm{H}$ and ${ }^{18} \mathrm{O}$, and tritium. Additional samples were collected from the six surface-water sites, plus four additional sites, during high flow (May 2007) and low flow (August 2006 and August 2007) and analyzed for CFCs and VOCs.

Maps showing the probability of groundwater contamination by various contaminants, or the likelihood of occurrence of young water, were developed using the logistic regression models. Before constructing the maps, all Geographic Information System (GIS) data were converted to grids with 10-meter (32.8-feet) spacing. The soils data required an additional processing step. To create the data set for logistic regression modeling, soils data were averaged within 500-meter (1,640-foot) pie-shaped buffers oriented upgradient from each well site. To create the final probability maps, the soils data within 500-meter (1,640-foot) pie-shaped buffers oriented upgradient from every grid cell in each soils layer/factor were averaged. After the soils data were averaged, then the logistic regression models were entered into a GIS, and a probability rating was calculated for each grid node in the study area.

The groundwater probability models were developed as follows: (1) The groundwater quality and groundwater age data were overlaid with anthropogenic and hydrogeologic data by using a geographic information system to produce a data set in which each well had corresponding data on depth to groundwater, distance to major streams and canals, distance to gypsum beds, precipitation, soils, and well depth. These data then were downloaded to a statistical software package for analysis by logistic regression. (2) Statistical models predicting the probability of elevated nitrate concentrations, the probability of unmixed young water (using chlorofluorocarbon-11 concentrations and tritium activities), and the probability of elevated volatile organic compound concentrations were developed using logistic regression techniques. (3) The statistical models were entered into a GIS and the probability maps were constructed.

The major-ion data indicated that groundwaters in the ERWVFA can be classified into two major groups: groundwater that was recharged by infiltration of surface water, and groundwater that had less immediate recharge from surface water and had elevated sulfate concentrations. Sulfate exceeded the U.S. Environmental Protection Agency National Secondary Drinking Water Regulations for sulfate (250 milligrams per liter $[\mathrm{mg} / \mathrm{L}])$ in many wells near Eagle, Gypsum, and Dotsero. The most significant source of sulfate to groundwater in the ERWVFA is the Eagle Valley Evaporite, which is a Pennsylvanian-age gypsum deposit located in the western half of Eagle County.
Many domestic well owners in the western portion of the watershed use reverse-osmosis water-treatment systems to reduce the amount of sulfate in their drinking water.

Nitrite plus nitrate as nitrogen (nitrate) concentrations in groundwater in the ERWVFA were low, with the median nitrate concentration about $0.74 \mathrm{mg} / \mathrm{L}$, and the maximum concentration measured $5.4 \mathrm{mg} / \mathrm{L}$. More than 50 percent of the nitrate concentrations in the ERWVFA were less than $1 \mathrm{mg} / \mathrm{L}$, indicating that more than 50 percent of the wells tested in the ERWVFA had nitrate concentrations similar to natural recharge water (precipitation). Most groundwater in the ERWVFA was under oxidized geochemical conditions, indicating that nitrate from anthropogenic sources (caused or produced by humans) could persist for several decades in the groundwater of the ERWVFA.

Apparent groundwater ages were estimated from the CFC and tritium data by using models that assume either piston flow or binary mixing (dilution of a young component with old, tracer-free water). The median groundwater recharge date was 1989 and the standard deviation was about 9 years, indicating that most groundwater in the ERWVFA was young water. The median percentage of young water was 83 percent and the standard deviation was about 28 percent, indicating that only a small portion of water from most wells was composed of old (older than 1940) water. To put the groundwater age data in the context of the probability of groundwater contamination, most groundwater in the ERWVFA was recently recharged water and had a high predisposition to contamination by anthropogenic compounds.

VOCs were detected in all water samples at the low-level minimum detection level concentrations, but all VOC concentrations were at least one order of magnitude less than their U.S. Environmental Protection Agency Maximum Contaminant Level. Although VOCs are commonly associated with anthropogenic sources, several VOCs are produced naturally in the environment, and, if not degraded or otherwise altered, can be found in water that was not affected by human sources. A useful indicator of VOC concentrations larger than those produced naturally in the environment is to sum the total concentrations of halogenated VOCs. Water from wells in the ERWVFA with total VOC concentrations greater than 28,000 picograms per liter $(\mathrm{pg} / \mathrm{L})$ probably had total VOC concentrations larger than that produced naturally in the environment. Wells with total VOC concentrations greater than $28,000 \mathrm{pg} / \mathrm{L}$ can be used to identify areas that had a high predisposition to anthropogenic contamination by VOCs.

Logistic regression statistical modeling techniques were used to develop three statistical models that predict the probability of groundwater contamination by various contaminants. These three probability models used different compounds such as nitrate and VOCs to provide an indication of the predisposition to groundwater contamination by anthropogenic compounds under a variety of conditions and contaminant inputs. These three models predict the probability of elevated nitrate concentrations, the probability of unmixed young water (using CFC-11 concentrations and tritium activities), and the probability of elevated VOC concentrations. 
The statistical parameters produced by the three logistic regression models indicated they were statistically significant models. McFadden's rho ranged between 0.490 and 0.647 , and the total correct predictions ranged between 0.778 and 0.876 , meaning they were highly effective models. The logistic regression models were verified by plotting the percentage of actual detections with the predicted probability of detections using a deciles of risk calculation. R-squared values of plots of actual detections with the predicted probability of detections were between 0.995 and 0.998 , verifying they were highly effective models.

Although the results of the groundwater age dating indicated that most areas in the ERWVFA had a high predisposition to contamination because most waters had groundwater recharge dates younger than 1980, the three probability models further delineated the probability of contamination for three different classes of compounds. The nitrate probability model showed the relative risk of elevated nitrate concentrations in groundwater from sources of nitrate such as fertilizers, manure, and wastewater. The entire ERWVFA had a predisposition to nitrate contamination from fertilizers, manure, and wastewater, but areas with larger depth to groundwater and less transmissive soils had a greater risk. The model predicting the probability of unmixed young water in the ERWVFA can be considered a generic model that is applicable for most contaminants. If a spill were to occur on the land surface or into the local surface water, those contaminants could be transported to the groundwater along the same pathways that the young recharge water uses. Although the entire ERWVFA had a predisposition to VOC contamination to some degree, the VOC probability map indicates that the areas near Vail, Avon, and Edwards had the highest probability of groundwater contamination if a spill should occur. Based upon the high occurrence of VOC detections observed by this study, VOC contamination is a concern in the ERWVFA.

\section{Acknowledgments}

The authors thank Ray Merry of the Eagle County Department of Environmental Health for his support and guidance throughout this study. The authors thank Kirby Wynn, formerly of the USGS, who built this study from the ground up and who developed the strong relationships with all the stakeholders involved. The authors thank Marcella Hutchinson and Mike Wireman of the U.S. Environmental Protection Agency for their support to get this project started. The authors thank the many landowners who permitted the use of their wells for collection of water samples, and the staff of the Eagle River Water and Sanitation District for access to their wells to collect groundwater-quality samples. The authors thank Jeff Starn of the USGS, who helped with the Monte Carlo particle-tracking simulations, Sharon Qi of the USGS, who helped us to calculate the pie-shaped zones of influence, Jason Gurdak of the USGS, who helped with the particle-tracking modeling, and Michael Stevens of the USGS who helped with the groundwater sampling. The authors thank Jason Gurdak and Tom Nolan of the USGS for their review comments.

\section{References Cited}

Aller, Linda, Bennett, Truman, Lehr, J.H., and Petty, R.J., 1985, DRASTIC — A standardized system for evaluating ground water pollution potential using hydro-geologic settings: U.S. Environmental Protection Agency, Robert S. Kerr Environmental Research Laboratory, Office of Research and Development, EPA/600/2-85/018, 163 p.

Apodaca, L.E., Driver, N.E., Stephens, V.C., and Spahr, N.E., 1996, Environmental setting and implications on water quality, Upper Colorado River Basin, Colorado and Utah: U.S. Geological Survey Water-Resources Investigations Report 95-4263, 33 p.

Barbash, J.E., and Resek, E.A., 1996, Pesticides in ground water-Distribution, trends, and governing factors: Chelsea, Mich., Ann Arbor Press, Inc., 588 p.

Barber, L.B., Thurman, E.M., Schroeder, M.P., LeBlanc, D.R., 1988, Long-term fate of organic micropollutants in sewagecontaminated groundwater: Environmental Science and Technology, v. 22, p. 205-211.

Bell, N.F., Hsu, L., Jacob, D..J., Schultz, M.G., Blake, D.R., Butler, J.H., King, D.B., Lobert, J.M., and Maier-Reimer, E., 2002, Methyl iodide-Atmospheric budget and use as a tracer of marine convection in global models: Journal of Geophysical Research, v. 107, no. D17, 4340, doi:10.1029/2001JD001151,2002.

Bouwer, Herman, 1978, Groundwater hydrology: New York, McGraw-Hill Book Company, 480 p.

Busenberg, Eurybiades, and Plummer, L.N., 1992, Use of chlorofluoromethanes $(\mathrm{CCl} 3 \mathrm{~F}$ and $\mathrm{CCl} 2 \mathrm{~F} 2)$ as hydrologic tracers and age-dating tools-Example, The alluvium and terrace system of central Oklahoma: Water Resources Research, v. 28, no. 9, p. 2257-2283.

Carter, J.M., Lapham, W.W., and Zogorski, J.S., 2008, Occurrence of volatile organic compounds in aquifers of the United States: Journal of the American Water Resources Association, v. 44, no. 2, p. 399-416.

Chuck, A.L., Turner, S.M., and Liss, P.S., 2005, Oceanic distributions and air-sea fluxes of biogenic halocarbons in the open ocean: Journal of Geophysical Research, v. 110, no. C10022, doi:10.1029/2004JC002741.

Clark, I.D., and Fritz, Peter, 1997, Environmental isotopes in hydrogeology: New York, Lewis Publishers, 328 p.

Connor, B.F., Rose, D.L., Noriega, M.C., Murtagh, L.K., and Abney, S.R., 1998, Methods of analysis by the U.S. Geological Survey National Water Quality LaboratoryDetermination of 86 volatile organic compounds in water by gas chromatography/mass spectrometry, including detections less than reporting limits: U.S. Geological Survey Open-File Report 97-829, 78 p. 
Cook, P.G., and Böhlke, J.K., 2000, Determining timescales for groundwater flow and solute transport, in Cook, P. G., and Herczeg, A., eds., Environmental tracers in subsurface hydrology: Boston, Kluwer Academic Publishers, p. 1-30.

Coplen, T.B., Wildman, J.D. and Chen, J., 1991, Improvements in the gaseous hydrogen-water equilibration technique for hydrogen isotope ratio analysis: Analytical Chemistry, v. 63, p. 910-912.

Cox, M.L., Sturrock, G.A., Fraser, P.J., Siems, S.T., and Krummel, P.B., 2005, Identification of regional sources of methyl bromide and methyl iodide from AGAGE observations at Cape Grim, Tasmania: Journal or Atmospheric Chemistry, v. 50, no. 1, p. 59-77.

Daly, Christopher, Neilson, R.P., and Phillips, D.L., 1994, A statistical-topographic model for mapping climatological precipitation over mountainous terrain: Journal of Applied Meteorology, v. 33, p. 140-158, accessed May 7, 2008, at http://www.prism.oregonstate.edu/pub/ prism/docs/jappclim94-modeling_mountain_precip-daly. $p d f$. Geospatial data accessed January 31, 2001, at http://www.prism.oregonstate.edu/products/.

Day, W.C., Knepper, D.H., Jr., Green, G.N., and Phillips, R.C., 1999, Spatial geologic data model for the Gunnison, Grand Mesa, Uncompahgre National Forests Mineral Resource Assessment Area, southwestern Colorado and digital data for the Leadville, Montrose, Durango, and Colorado parts of the Grand Junction, Moab, and Cortez $1^{\circ} \times 2^{\circ}$ geologic maps: U.S. Geological Survey Open-File Report 99-427, accessed December 2007, at http://pubs.usgs.gov/of/1999/ of $-99-0427 /$.

Druliner, A.D., Chen, H.H., and McGrath, T.S., 1996, Relations of nonpoint-source nitrate and atrazine concentrations in the High Plains aquifer to selected explanatory variables in six Nebraska study areas: U.S. Geological Survey WaterResources Investigations Report 95-4202, 51 p.

Eckhardt, D.A.V., and Stackelberg, P.E., 1995, Relation of ground-water quality to land use on Long Island, N.Y.: Ground Water, v. 33, no. 6, p. 1019-1033.

Epstein, S., and Mayeda, T., 1953, Variation of O-18 content of water from natural sources: Geochimica et Cosmochimica Acta, v. 4, p. 213-224.

Fishman, M.J., ed., 1993, Methods of analysis by the U.S. Geological Survey National Water Quality LaboratoryDetermination of inorganic and organic constituents in water and fluvial sediments: U.S. Geological Survey Open-File Report 93-125.

Fishman, M.J., and Friedman, L.C., 1989, Methods for determination of inorganic substances in water and fluvial sediments: U.S. Geological Survey Techniques of WaterResources Investigations, book 5, chap. A1, 545 p.
Focazio, M.J., Reilly, T.E., Rupert, M.G., and Helsel, D.R., 2002, Assessing ground-water vulnerability to contaminationProviding scientifically defensible information for decision makers: U.S. Geological Survey Circular 1224, 33 p.

Forcada, E.G., and Evangelista, I.M., 2008, Contributions of boron isotopes to understanding the hydrogeochemistry of the coastal detritic aquifer of Castellón Plain, Spain: Hydrogeology Journal, v. 16, no. 3, p. 547-557.

Ford, M., and Tellam, J.H., 1994, Source, type and extent of inorganic contamination within the Birmingham urban aquifer system, UK: Journal of Hydrology, v. 156, nos. 1-4, p. 101-135.

Freeze, R.A., and Cherry, J.A., 1979, Groundwater: Englewood Cliffs, N.J., Prentice-Hall, 604 p.

Gallant, A.L., Whittier, T.R., Larsen, D.P., Omernick, J.M., and Hughes, R.M., 1989, Regionalization as a tool for managing environmental resources: U.S. Environmental Protection Agency Report EPA/600/3-89/060, 152 p.

Giegengack, R.F., 1962, Recent volcanism near Dotsero, Colorado: Boulder, University of Colorado, unpublished Master's thesis, $43 \mathrm{p}$.

Gribble, G.W., 1994, The natural production of chlorinated compounds: Environmental Science \& Technology, v. 28, no. 7 , p. 310A-319A.

Gurdak, J.J., 2008, Ground-water vulnerability-Nonpointsource contamination, climate variability, and the High Plains aquifer: Saarbrucken, Germany, VDM Verlag Publishing, ISBN: 978-3-639-09427-5, 223 p.

Gurdak, J.J., McCray, J.E., Thyne, G.D., and Qi, S.L., 2007, Latin hypercube approach to estimate uncertainty in ground water vulnerability: Ground Water, v. 45, no. 3, p. 348-361, doi: 10.1111/j.1745-6584.2006.00298.x.

Gurdak, J.J., and Qi, S.L., 2006, Vulnerability of recently recharged ground water in the High Plains aquifer to nitrate contamination: U.S. Geological Survey Scientific Investigations Report 2006-5050, 39 p.

Harbaugh, A.W., Banta, E.R., Hill, M.C., and McDonald, M.G., 2000, MODFLOW-2000, The U.S. Geological Survey modular ground-water model-User guide to modularization concepts and the ground-water flow process: U.S. Geological Survey Open-File Report 2000-92, 121 p.

Helsel, D.R., and Hirsch, R.M., 1992, Statistical methods in water resources: New York, Elsevier Science Publishing Company, Inc., 522 p.

Hosmer, D.W., and Lemeshow, Stanley, 1989, Applied logistic regression: New York, John Wiley \& Sons, Inc., 307 p. 
International Atomic Energy Agency (IAEA), 2006, Use of chlorofluorocarbons in hydrology-A guidebook: Vienna, Austria, International Atomic Energy Agency, STI/PUB/1238, ISBN 92-0-100805-8, 277 p., accessed November 11, 2008, at http://www-pub.iaea.org/MTCD/ publications/PDF/Pub1238_web.pdf.

Isidorov, V.A., Zenkevich, I.G., and Ioffe, B.V., 1990, Volatile organic compounds in solfataric gases: Journal Atmospheric Chemistry, v. 10, no. 3, p. 329-340.

Keene, W.C., Khalil, M.A.K., Erickson III, D.J., McCulloch, Archie, Graedel, T.E., Lobert, J.M., Aucott, M.L., Gong, S.L., Harper, D.B., Kleiman, Gary, Midgley, Pauline, Moore, R.M., Seuzaret, Christophe, Sturges, W.T., Benkovitz, C.M., Koropalov, Valentin, Barrie, L.A., and Li, Y.F., 1999, Composite global emissions of reactive chlorine from anthropogenic and natural sources-Reactive chlorine emissions inventory: Journal of Geophysical Research, v. 104, no. D7, p. 8429-8440.

Khalil, M.A.K., Moore, R.M., Harper, D.B., Lobert, J.M., Erickson, D.J., Koropalov, V., Sturges, W.T., and Keene, W.C., 1999, Natural emissions of chlorine-containing gases-Reactive chlorine emissions inventory: Journal of Geophysical Research, v. 104, no. D7, p. 8333-8346.

Kleinbaum, D.G., 1994, Logistic regression, a self-learning text: New York, Springer-Verlag, 282 p.

Koterba, M.T., Banks, W.S.L., and Shedlock, R.J., 1993, Pesticides in shallow groundwater in the Delmarva Peninsula: Journal of Environmental Quality, v. 22, no. 3 , p. $500-518$.

Koterba, M.T., Wilde, F.D., and Lapham, W.W., 1995, Ground-water data-collection protocols and procedures for the National Water-Quality Assessment ProgramCollection and documentation of water-quality samples and related data: U.S. Geological Survey Open-File Report 95-399, 113 p.

Laturnus, Frank, Haselmann, K.F., Borch, Thomas, and Gron, Christian, 2002, Terrestrial natural sources of trichloromethane (chloroform, $\mathrm{CHCl} 3$ ) - An overview: Biochemistry, v. 60, no. 2, p. 121-139.

Leenheer, J.A., Rostad, C.E., Barber, L.B., Schroeder, R.A., Anders, R., and Davisson, M.L., 2001, Nature and chlorine reactivity of organic constituents from reclaimed water in groundwater, Los Angeles County, California: Environmental Science and Technology, v. 35, p. 3869-3876.

Leenhouts, J.M., Bassett, R.L., and Maddock III, T., 1998, Utilization of intrinsic boron isotopes as co-migrating tracers for identifying potential nitrate contamination sources: Ground Water, v. 36, no. 2, p. 240-250.
Lidke, D.J., 1998, Geologic map of the Wolcott quadrangle, Eagle County, Colorado: U.S. Geological Survey Geologic Investigations Series I-2656, accessed December 2007 at http://greenwood.cr.usgs.gov/pub/i-maps/i-2656/i2656.pdf.

Lorenz, D.L., Goldstein, R.M., Cowdery, T.K., and Stoner, J.D., 2003, Comparison of two methods for delineating land use near monitoring wells used for assessing quality of shallow ground water: U.S. Geological Survey Water-Resources Investigations Report 2003-4067, 13 p.

Lucas L.L., and Unterweger, M.P., 2000, Comprehensive review and critical evaluation of the half-life of tritium: Journal of Research of the National Institute of Standards and Technology, v. 105, no. 4, p. 541-549.

Małoszewski, Piotr, and Zuber, Andrzej, 1982, Determining the turnover time of groundwater systems with the aid of environmental tracers. 1. Models and their applicability: Journal of Hydrology, v. 57, p. 207-231.

Maloszewski, Piotr, and Zuber, Andrzej, 1996, Lumped parameter models for the interpretation of environmental tracer data, in International Atomic Energy Agency, Manual on mathematical models in isotope hydrology: Vienna, Austria, IAEA-TECDOC-910, p. 9-58.

Małoszewski, Piotr, Rauert, Werner, Stichler, Wili, and Herrmann, Andreas, 1983, Application of flow models to an alpine catchment area using tritium and deuterium data: Journal of Hydrology, v. 66, p. 319-330.

McCulloch, Archie, 2003, Chloroform in the environmentOccurrence, sources, sinks and effects: Chemosphere, v. 50, no. 10, p. 1291-1308.

McMahon, P.B., Dennehy, K.F., Bruce, B.W., Gurdak, J.J., and Qi, S.L., 2007, Water-quality assessment of the High Plains aquifer, 1999-2004: U.S. Geological Survey Professional Paper 1749, $136 \mathrm{p}$.

Moran, M.J., Hamilton, P.A., and Zogorski, J.S., 2006, Volatile organic compounds in the Nation's ground water and drinking-water supply wells-A summary: U.S. Geological Survey Fact Sheet FS 2006-3048, 6 p.

Naftz, D.L., Bullen, T.D., Stolp, B.J., and Wilkowske, C.D., 2008, Utilizing geochemical, hydrologic, and boron isotopic data to assess the success of a salinity and selenium remediation project, Upper Colorado River Basin, Utah: Science of the Total Environment v. 392, no. 1, p. 1-11.

National Atmospheric Deposition Program, 2007, Welcome to NADP: National Atmospheric Deposition Program, NADP Brochure 2007-01, 2 p., accessed March 5, 2008 at http://nadp.sws.uiuc.edu/lib/brochures/welcomebrochure.pdf. 
National Research Council, 1993, Ground water vulnerability assessment, contamination potential under conditions of uncertainty: National Academy Press, Washington, D.C., 210 p., accessed December 15, 2000 from URL http://books.nap.edu/books/0309047994/html.

Nolan, B.T., and Clark, M.L., 1997, Selenium in irrigated agricultural areas of the western United States: Journal of Environmental Quality, v. 26, no. 3, p. 849-857.

Nolan, B.T., and Hitt, K.J., 2006, Vulnerability of shallow groundwater and drinking-water wells to nitrate in the United States: Environmental Science \& Technology, v. 40, no. 24 , p. $7834-7840$.

Nolan, B.T., and Hitt, K.J., 2002, Nutrients in shallow ground waters beneath relatively undeveloped areas in the conterminous United States: U.S. Geological Survey WaterResources Investigations Report 2002-4289, 17 p.

Nolan, B.T., Hitt, K.J., and Ruddy, B.C., 2002, Probability of nitrate contamination of recently recharged groundwaters in the conterminous United States: Environmental Science \& Technology, v. 36, no. 10, p. 2138-2145.

Paschke, S.S., ed., 2007, Hydrogeologic settings and groundwater flow simulations for regional studies of the transport of anthropogenic and natural contaminants to public-supply wells - studies begun in 2001: U.S. Geological Survey Professional Paper 1737-A, 244 p.

Piper, A.M., 1944, A graphic procedure in the geochemical interpretation of water analyses: Transactions, American Geophysical Union, v. 25, p. 914-923.

Plummer, L.N., Böhlke, J.K., and Busenberg, Eurybiades, 2003, Approaches for ground-water dating, in Lindsey, B.D., Phillips, S.W., Donnelly, C.A., Speiran, G.K., Plummer, L.N., Böhlke, J.K., Focazio, M.J., Burton, W.C., and Busenberg, Eurybiades, eds., Residence times and nitrate transport in ground water discharging to streams in the Chesapeake Bay watershed: U.S. Geological Survey Water-Resources Investigations Report 2003-4035, p. 12-24.

Plummer, L.N., and Busenberg, Eurybiades, 1999, Chlorofluorocarbons-Tools for dating and tracing young groundwater, in, Cook, P., and Herczeg, A., eds., Environmental tracers in subsurface hydrology: P. Boston, Kluwer Academic Publishers, chap. 15, p. 441-478.

Plummer, L.N., Busenberg, Eurybiades, and Cook, P.G., 2006, Principles of chlorofluorocarbon dating, in International Atomic Energy Agency, Use of chlorofluorocarbons in hydrology — A guidebook: Vienna, International Atomic Energy Agency, p. 17-29.
Plummer, L.N., Busenberg, Eurybiades, Eberts, S.M., Bexfield, L.M., Brown, C.J., Fahlquist, L.S., Katz, B.G., and Landon, M.K., 2008, Low-level detections of halogenated volatile organic compounds in groundwater-Use in vulnerability assessments: Journal of Hydrologic Engineering, v. 13, no. 11, p. $1049-1068$.

Plummer, L.N., Busenberg, Eurybiades, and Widman, P.K., 2004, Applications of dissolved N2 and Ar in groundwater: Geological Society of America, Abstracts with Programs, v. 36 , no. 5 , p. 468 .

Plummer, L.N., and Friedman, L.C., 1999, Tracing and dating young ground water: U.S. Geological Survey Fact Sheet FS-134-99.

Plummer, L.N., Michel, R.L., Thurman, E.M., and Glynn, P.D., 1993, Environmental tracers for age dating young ground water, in Alley, W.M., ed., Regional ground-water quality: N.Y., Van Nostrand Reinhold, p. 255-294.

Pollock, D.W., 1994, User's guide for MODPATH/MODPATHPLOT, Version 3-A particle tracking post-processing package for MODFLOW, the U.S. Geological Survey finitedifference ground-water flow model: U.S. Geological Survey Open-File Report 94-464, 236 p.

Princeton Geoscience, 2005, Road salting as a cause of chloroform in ground water?: accessed November 18, 2005, at http://princetongeoscience.com/geonew.html.

Rao, P.S.C., and Alley, W.M., 1993, Pesticides: N.Y., Van Nostrand Reinhold, 112 p.

Reilly, T.E., and Harbaugh, A.W., 2004, Guidelines for evaluating ground-water flow models: U.S. Geological Survey Scientific Investigations Report 2004-5038, 30 p.

Rose, D.L., and Schroeder, M.P., 1995, Methods of analysis by the U.S. Geological Survey National Water Quality Laboratory-Determination of volatile organic compounds in water by purge and trap capillary gas chromatography/ mass spectrometry: U.S. Geological Survey Open-File Report 94-708, 26 p.

Rupert, M.G., 1998, Probability of detecting atrazine/ desethyl-atrazine and elevated concentrations of nitrate $(\mathrm{NO} 2+\mathrm{NO} 3-\mathrm{N})$ in ground water in the Idaho part of the upper Snake River Basin: U.S. Geological Survey WaterResources Investigations Report 98-4203, 32 p., 1 pl., accessed March 10, 2001, at http://idaho.usgs.gov/PDF/ wri984203/index.html. 
Rupert, M.G., 2001, Calibration of the DRASTIC ground water vulnerability mapping method: Ground Water, v. 39, no. 4, p. 625-630.

Rupert, M.G., 2003, Probability of detecting atrazine/ desethyl-atrazine and elevated concentrations of nitrate in ground water in Colorado: U.S. Geological Survey WaterResources Investigations Report 2002-4269, 35 p.

Rupert, M.G., and Plummer, L.N., 2004, Ground-water flow direction, water quality, recharge sources, and age, Great Sand Dunes National Monument, south-central Colorado, 2000-2001: U.S. Geological Survey Scientific Investigations Report 2004-5027, 28 p.

Schwarz, G.E., and Alexander, R.B., 1995, State Soil Geographic (STATSGO) data base for the conterminous United States: U.S. Geological Survey Open-File Report 95-449, accessed March 10, 2006, at http://water.usgs.gov/lookup/ getspatial? ussoils.

Shapiro, S.D., Plummer, L.N., Focazio, M.J., Busenberg, E., Kirkland, W., and Fernandez, M., 2003, Occurrence of volatile organic compounds in drinking water from the United States-Results from archived chromatograms and water samples, 1989-2000: U.S. Geological Survey WaterResources Investigations Report 2002-4173, 20 p.

Starn, J.J., Stone, J.R., and Mullaney, J.R., 2000, Delineation and analysis of uncertainty of contributing areas to wells at the Southbury Training School, Southbury, Connecticut: U.S. Geological Survey Water-Resources Investigations Report 2000-4158, 54 p.

State of Colorado, 2005, Rules and regulations for water well construction, pump installation, cistern installation, and monitoring and observation hole/well constructions: State of Colorado, Office of the State Engineer, 2 CCR 402-2, 54 p., accessed November 2007, at http://water.state.co.us/boe/ rulesregs/constructionrules05.pdf.

Sturges, W.T., McIntyre, H.P., Penkett, S.A., Chappellaz, J., Barnola, J.M., Mulvaney, R., Atlas, E., and Stroud, V., 2001, Methyl bromide, other brominated methanes, and methyl iodide in polar firn air: Journal of Geophysical Research, v. 106, no. D2, p. 1595-1606.

SYSTAT Software, Inc., 2004, SYSTAT 11, Statistics IISoftware documentation: Richmond, Calif., SYSTAT Software, Inc., $657 \mathrm{p}$.

Tesoriero, A.J., and Voss, F.D., 1997, Predicting the probability of elevated nitrate concentrations in the Puget Sound Basin-Implications for aquifer susceptibility and vulnerability: Ground Water, v. 35, no. 6, p. 1029-1039.

Thatcher, L.L., Janzer, V.J., and Edwards, K.W., 1977, Methods for determination of radioactive substances in water and fluvial sediments: U.S. Geological Survey Techniques of Water-Resources Investigations, book 5, chap. A-5, 95 p.
Thurman, E.M., Barber, L.B., Jr., and LeBlanc, D., 1986, Movement and fate of detergents in groundwater-A field study: Journal of Contaminant Hydrology, v. 1 p. 143-161.

U.S. Census Bureau, 2008, State and county quickfacts: U.S. Census Bureau State \& County Quickfacts, accessed October 22, 2008, at http://quickfacts.census.gov/qfd/ states/08/08037.html.

U.S. Department of Agriculture, 1991, State Soil Geographic (STATSGO) data base: U.S. Department of Agriculture, Soil Conservation Service, Miscellaneous Publication 1492, $88 \mathrm{p}$.

U.S. Department of Agriculture, 1999, Holy Cross Area Soil Survey: United States Department of Agriculture, Forest Service, White River National Forest, Glenwood Springs, Colo., accessed March 2008 at http://www.fs.Fed.us/r2/whiteriver/ projects/gis.shtml.

U.S. Department of Agriculture, 2008, Soil Survey Geographic (SSURGO) database for Aspen-Gypsum area, parts of Eagle, Garfield and Pitkin Counties, Colorado: United States Department of Agriculture, Natural Resources Conservation Service, accessed July 23, 2008, at http://soildatamart.nrcs.usda.gov/ Metadata.aspx? Survey $=C O 655 \&$ UseState $=C O$.

U.S. Environmental Protection Agency, 1993, A review of methods for assessing aquifer sensitivity and ground water vulnerability to pesticide contamination: U.S. Environmental Protection Agency, EPA/813/R-93/002, 147 p.

U.S. Environmental Protection Agency, 1999, Health effects from exposure to high levels of sulfate: U.S. Environmental Protection Agency, EPA 815-R-99-001, 25 p., accessed December 2007 at http://www.epa.gov/safewater/ contaminants/unregulated/pdfs/study_sulfate_epa-cdc.pdf.

U.S. Environmental Protection Agency, 2003, Drinking water contaminants: U.S. Environmental Protection Agency, Office of Water, EPA 816-F-03-016, accessed July 28, 2008, at http://www.epa.gov/safewater/contaminants/index.html.

U.S. Geological Survey, variously dated, National field manual for the collection of water-quality data: U.S. Geological Survey Techniques of Water-Resources Investigations, book 9, chaps. A1-A9, accessed December 2008, at http://pubs.water.usgs.gov/twri9A.

U.S. Geological Survey, 1999a, National elevation dataset: U.S. Geological Survey Fact Sheet 148-99, 2 p., accessed July 22, 2008, at http://erg.usgs.gov/isb/pubs/factsheets/ fs 14899.html.

U.S. Geological Survey, 1999b, The National Hydrography Dataset: U.S. Geological Survey Fact Sheet 106-99, 2 p., accessed July 22, 2008, at http://erg.usgs.gov/isb/pubs/ factsheets/fs 10699.html. 
U.S. Geological Survey, 2001, USGS GeoData Digital Raster Graphics: U.S. Geological Survey Fact Sheet 088-01, 2 p., accessed on July 22, 2008, at http://erg.usgs.gov/isb/pubs/ factsheets/fs08801.html.

U.S. Geological Survey, 2007a, The Reston Chlorofluorocarbon Laboratory: accessed May 6, 2007, at http://waterusgs.gov/lab/.

U.S. Geological Survey, 2007b, The Reston Stable Isotope Laboratory: accessed May 6, 2007, at http://isotopes.usgs.gov/.

Vengosh, Avner, Heumann, K.G., Juraske, S., and Kasher, R., 1994, Boron isotope application for tracing sources of contamination in groundwater: Environmental Science and Technology, v. 28, p. 1968-1974.

Vengosh, Avner, and Pankratov, Irena, 1998, Chloride/bromide and chloride/fluoride ratios of domestic sewage effluents and associated contaminated ground water: Ground Water, v. 36 , no. 5 , p. $815-824$.

Verstraeten, I.M., Fetterman, G.S., Meyer, M.T., Bullen, T., and Sebree, S.K., 2005, Use of tracers and isotopes to evaluate vulnerability of water in domestic wells to septic waste: Ground Water Monitoring and Remediation, v. 25, no. 2 , p. 107-117.
Vowinkel, E.F., Clawges, R.M., Buxton, D.E., Stedfast, D.A., and Louis, J.B., 1996, Vulnerability of public drinking water supplies in New Jersey to pesticides: U.S. Geological Survey Fact Sheet FS-165-96, 3 p.

Walvoord, M.A., Phillips, F.M., Stonestrom, D.A., Evans, R.D., Hartsough, P.C., Newman, B.D., and Striegl, R.G., 2003, A reservoir of nitrate beneath desert soils: Science, v. 302, p. $1021-1024$.

Westgate, E.J., Kroeger, K.D., Pabich, W.J., and Valiela, Ivan, 2000, Fate of anthropogenic nitrogen in a nearshore Cape Cod aquifer: Biological Bulletin, v. 199, no. 2, p. 221-223.

Zuber, Andrzej, 1986, Mathematical models for the interpretation of environmental radioisotopes in groundwater systems, in Fritz, P., and Fontes, J. Ch., eds., Handbook of environmental isotope geochemistry: Amsterdam, Elsevier, v. 2, part B, p. 1-60.

Zuber, Andrzej, 1994, On calibration and validation of mathematical models for the interpretation of environmental isotope data in aquifers, in International Atomic Energy Agency, Mathematical models and their application to isotope studies in groundwater hydrology, Proceedings of a final research coordination meeting: Vienna, Austria, IAEA-TECHDOC-777, p. 11-41.

Publishing support provided by:

Denver Publishing Service Center, Denver, Colorado

Manuscript approved for publication, April 2, 2009

Edited by Mary A. Kidd

Graphics and layout by Joy Monson

For more information concerning this publication, contact:

Director, USGS Colorado Water Science Center

Box 25046, Mail Stop 415

Denver, CO 80225

(303) 236-4882

Or visit the Colorado Water Science Center Web site at:

http://co.water.usgs.gov/

This publication is available online at:

http://pubs.usgs.gov/sir/2009/5082/ 


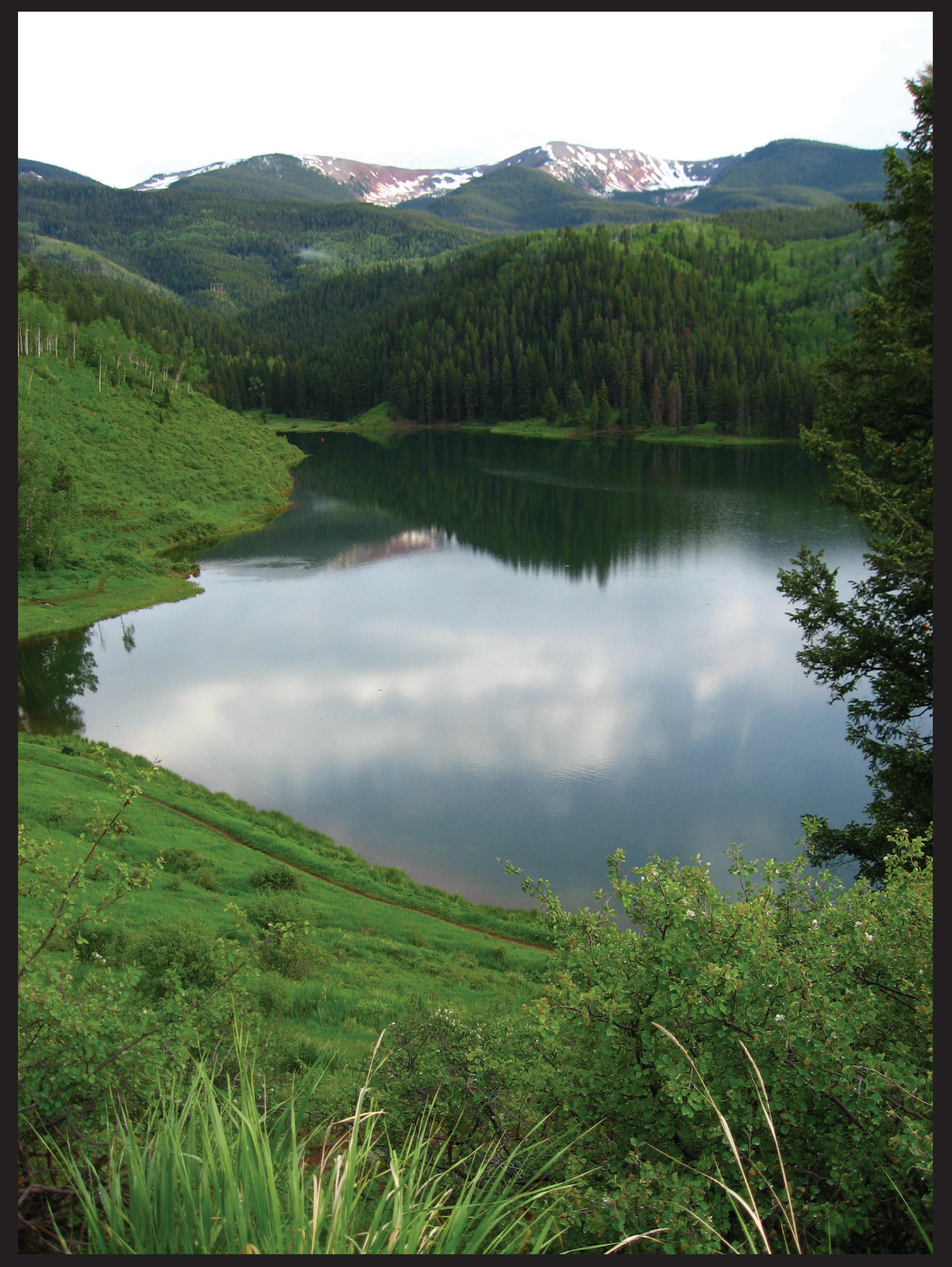

